\title{
On some time marching schemes for the stabilized finite element approximation of the mixed wave equation
}

\author{
Hector Espinoza ${ }^{a, b}$, Ramon Codina ${ }^{a, *}$, Santiago Badia ${ }^{a, c}$ \\ ${ }^{a}$ Universitat Politècnica de Catalunya, Jordi Girona 1-3, Edifici C1, E-08034 Barcelona, Spain \\ ${ }^{b}$ Escuela Superior Politécnica del Litoral ESPOL, Km 30.5 vía Perimetral, EC090902, Guayaquil, Ecuador \\ ${ }^{c}$ Centre Internacional de Mètodes Numèrics en Enginyeria, Parc Mediterrani de la Tecnologia, Esteve Terradas 5, E-08860 \\ Castelldefels, Spain
}

\begin{abstract}
In this paper we analyze time marching schemes for the wave equation in mixed form. The problem is discretized in space using stabilized finite elements. On the one hand, stability and convergence analyses of the fully discrete numerical schemes are presented using different time integration schemes and appropriate functional settings. On the other hand, we use Fourier techniques (also known as von Neumann analysis) in order to analyze stability, dispersion and dissipation. Numerical convergence tests are presented for various time integration schemes, polynomial interpolations (for the spatial discretization), stabilization methods, and variational forms. To analyze the behavior of the different schemes considered, a 1D wave propagation problem is solved.
\end{abstract}

Keywords: time marching schemes, dispersion, dissipation, von Neumann analysis, Fourier analysis, mixed wave equation, stabilized finite element methods, variational multiscale methods 2008 MSC: 35F46, 35L05, 35L50, 35L65, 35M13, 65J10, 65M12, 65M15, 65M60, 76M10

\section{Introduction}

Finite difference time marching schemes are mostly used for the time integration of evolution problems because of their efficiency and ease of implementation. In the case of partial differential equations (PDEs) in space and time, even if a given finite difference scheme has some general properties regarding stability and accuracy, the precise behavior of the scheme needs to be analyzed together with the spatial discretization employed. In this paper we aim at analyzing classical first and second order schemes for the hyperbolic wave equation, with the particularity that we write it in mixed form and discretize in space using stabilized finite element (FE) methods.

For the analysis of time discretization schemes for wave propagation problems it is customary to split the total discretization error into two parts: dispersion error and dissipation error. Dispersion is the dependency of the phase velocity on the frequency and the dispersion error is the deviation of the phase velocity with respect to the expected one for a given frequency. On the other hand, dissipation error is the decrease in amplitude with respect to the expected one. In general, dispersion and dissipation errors are higher for poorly resolved frequencies, which occurs mainly for high frequencies. The wave equation we aim to analyze is nondispersive and non-dissipative. Therefore, it would be desirable to have non-dispersive and non-dissipative discretization schemes. This sometimes cannot be achieved, thus one just aims to have low-dispersion lowdissipation schemes [1, 2]. Dispersion and/or dissipation of numerical schemes can be evaluated using Fourier

\footnotetext{
*Corresponding author. Tel.: +34 934016486

Email addresses: hector.espinoza@upc.edu (Hector Espinoza), ramon.codina@upc.edu (Ramon Codina), sbadia@cimne.upc.edu (Santiago Badia)

URL: https://upc.edu/espinoza (Hector Espinoza), http://codina.rmee.upc.edu/ (Ramon Codina), http://badia.rmee.upc.edu/ (Santiago Badia)
} 
techniques (see [3, 4, 5]), energy methods (see [6]), or modified equation analysis (see [4, 7]). Fourier analysis can be carried out for semi-discretizations or full discretizations. Dispersion/dissipation analysis methods have been used to optimize numerical schemes [3, 7. Other properties of the continuous wave equation, such as the preservation of symplecticity, some invariants or some symmetries are even more difficult to inherit for discrete schemes. This is the motivation of the so-called geometric numerical integrators, which we will not consider in this paper (see [8], for example).

Contrary to the irreducible hyperbolic wave equation, which is of second order in space and time, the mixed wave equation is of first order in space and time. We will consider the case of a single scalar unknown, which in the mixed format unfolds into two unknowns, namely, this scalar field and a vector unknown. With regard to the space approximation, the Galerkin FE discretization of this mixed wave equation requires to satisfy a compatibility condition between the spaces of the two unknowns (scalar and vector), i.e., to use so-called inf-sup compatible interpolations. Alternatively, we can consider stabilized FE methods, which provide much more flexibility when choosing the interpolation spaces [9, 10, 11. In particular, we can consider equal interpolation for the unknowns. Stability and convergence of the stabilized FE spatial semidiscretization of the mixed wave equation has been presented in [10, 11. Stability and convergence of fully discrete schemes has also been analyzed for the convection-diffusion equation and the Stokes equations in [12, 13, 14] using spatial and temporal approximations related to those used in the present work. Note that the use of stabilized FE methods for general first order hyperbolic equations is an old topic, which can be traced back to [15] if only the advective terms are considered. However, we exploit here the particular structure of the wave equation and the functional settings that can be associated to it; this allows us to go much further in the analysis.

In this work, we analyze the stability and convergence properties for the mixed wave equation, after time semi-discretization, space semi-discretization, and full discretization, and perform their Fourier analyses. For the time discretization, we consider backward Euler (BE), Crank-Nicolson ${ }^{1}(\mathrm{CN})$ and the second order backward differentiation formula (BDF2). We will see how a symplectic time integrator (CN) compares to non-symplectic time integrators (BE and BDF2). Dispersion and dissipation of discretization methods will be evaluated through numerical experiments. This consists in solving a given problem and evaluating the solution obtained [17, 18, 3. We will solve a 1D wave propagation problem to show qualitatively dispersion and dissipation of the proposed numerical schemes.

The organization of the paper is as follows. In Section 2 we present the problem statement and its spacetime discretization. In Section 3 we present stability and convergence results of the fully discrete problem obtained using variational techniques. We provide results for all the methods considered, even though we only present one sample of the proofs of these results. In Section 4 we present a complete Fourier analysis for the 1D wave equation in mixed form, from which precise information on the behavior of the different schemes can be drawn. Numerical results are presented in Section 5 and, finally, in Section 6 the conclusions of the work are summarized. This paper is a continuation of our work on the approximation of the mixed form of the wave equation presented in [10, 11]. Frequent reference is made to these two papers, to which the reader is addressed for details.

\section{Problem statement and numerical approximation}

\subsection{Initial and boundary value problem}

The problem we consider is an initial and boundary value problem posed in a time interval $\Upsilon:=(0, T)$ and in a spatial domain $\Omega \subset \mathbb{R}^{d},(d=1,2$ or 3$)$. Let $t \in \Upsilon$ be a given time instant in the temporal domain and $\boldsymbol{x} \in \Omega$ a given point in the spatial domain. We define the space-time domain as $\Xi:=\Omega \times \Upsilon$. Let $\Gamma$ be the boundary of the domain $\Omega$. We split $\Gamma$ into three disjoint sets denoted as $\Gamma_{p}, \Gamma_{u}$ and $\Gamma_{\mathrm{o}}$. The scalar

\footnotetext{
${ }^{1}$ The original CN discretization scheme was devised to solve numerically PDEs of heat-conduction type; it is a space-time discretization based on finite differences. Sometimes CN is used to refer to the implicit midpoint method or the (implicit) trapezoidal rule and there is no agreement in the literature [16. We have to mention that for linear operators (which is the the case of the mixed wave equation) the trapezoidal rule and the implicit midpoint method are equivalent.
} 
unknown $p$ is enforced on $\Gamma_{p}$, the normal trace of the vector unknown $\gamma_{n} \boldsymbol{u}$ on $\Gamma_{u}$, and a simple non reflecting boundary condition (NRBC) on $\Gamma_{\mathrm{o}}$. Although the boundary conditions are irrelevant for the von Neumann analysis, we just mention them for completeness.

The problem consists in finding $p: \Xi \longrightarrow \mathbb{R}$ and $\boldsymbol{u}: \Xi \longrightarrow \mathbb{R}^{d}$ such that

$$
\begin{aligned}
\mu_{p} \partial_{t} p+\nabla \cdot \boldsymbol{u} & =f_{p}, \\
\mu_{u} \partial_{t} \boldsymbol{u}+\nabla p & =\boldsymbol{f}_{u},
\end{aligned}
$$

with the following initial conditions

$$
p(\boldsymbol{x}, 0)=0, \quad \boldsymbol{u}(\boldsymbol{x}, 0)=\mathbf{0},
$$

and with the following boundary conditions

$$
p=0 \quad \text { on } \Gamma_{p}, \quad \gamma_{n} \boldsymbol{u}:=\boldsymbol{n} \cdot \boldsymbol{u}=0 \quad \text { on } \Gamma_{u}, \quad \mu_{p}^{\frac{1}{2}} p=\mu_{u}^{\frac{1}{2}} \gamma_{n} \boldsymbol{u} \text { on } \Gamma_{\mathrm{o}},
$$

where $\mu_{p}>0$ and $\mu_{u}>0$ are physical coefficients such that $c^{2}=\left(\mu_{p} \mu_{u}\right)^{-1}, c$ is the wave speed, $f_{p}$ and $\boldsymbol{f}_{u}$ are forcing terms, $\boldsymbol{n}$ is the unit outward normal to the boundary of the domain and $\gamma_{n}$ is the normal trace operator. In the previous equations and in what follows, we use the following convention: lower-case bold italic letters represent vectors in $\mathbb{R}^{d}$, lower-case non-bold italic letters represent scalars, whereas upper-case non-bold italic letters may be arrays or matrices.

Let $\Psi$ be a generic spatial domain, i.e. $\Omega$ or $\Gamma$ or part of them. Whenever they are well defined, we denote by $L^{2}(\Psi)$ the space of square integrable functions defined on $\Psi$, by $H^{1}(\Psi)$ the space of functions in $L^{2}(\Psi)$ with derivatives in $L^{2}(\Psi)$, by $H(\operatorname{div}, \Psi)$ the space of vector functions with components and divergence in $L^{2}(\Psi)$, and by $L^{2}(\Psi)^{d}$ the space of vector functions with components in $L^{2}(\Psi)$. Additionally, for an arbitrary normed functional space $\mathrm{X}$, its norm will be denoted as $\|\cdot\|_{\mathrm{X}}$. In the case of $L^{2}(\Omega)$ or $L^{2}(\Omega)^{d}$ the $L^{2}$-norm will simply be denoted as $\|\cdot\|$ and the $L^{2}$-inner-product as $(\cdot, \cdot)$ Furthermore, the space of functions whose $\mathrm{X}$-norm is $C^{r}$ continuous in the time interval $\Upsilon$ will be denoted by $C^{r}(\Upsilon ; \mathrm{X})$. We will only be interested in the cases $r=0$ and $r=1$. Functions whose X-norm is $L^{p}$ in $\Upsilon$ will be denoted by $L^{p}(\Upsilon ; \mathrm{X})$; when $\mathrm{X}=L^{2}(\Omega)$ or $\mathrm{X}=L^{2}(\Omega)^{d}$, the compact notation $L^{p}\left(L^{2}\right)$ will sometimes be used. Furthermore, let $\mathrm{V}_{p}, \mathrm{~V}_{u}$ be spaces associated with $p$ and $\boldsymbol{u}$ respectively. These spaces will be defined afterwards because they depend on the functional setting. Additionally, let us define $\mathrm{V}:=\mathrm{V}_{p} \times \mathrm{V}_{u}$ and $\mathrm{L}:=L^{2}(\Omega) \times L^{2}(\Omega)^{d}$.

Problem (1)-(2) with appropriate initial and boundary conditions will be well-posed for:

$$
p \in C^{1}\left(\Upsilon ; L^{2}(\Omega)\right) \cap C^{0}\left(\Upsilon ; \mathrm{V}_{p}\right), \quad \boldsymbol{u} \in C^{1}\left(\Upsilon ; L^{2}(\Omega)^{d}\right) \cap C^{0}\left(\Upsilon ; \mathrm{V}_{u}\right),
$$

with $f_{p}$ and $\boldsymbol{f}_{u}$ regular enough.

\subsection{Variational problem}

The variational form of problem (1)-(4) can be expressed in three different ways. Each one requires a certain regularity on the unknowns $p$ and $\boldsymbol{u}$. The problem reads: find $[p, \boldsymbol{u}] \in C^{1}(\Upsilon ; \mathrm{L}) \cap C^{0}(\Upsilon ; \mathrm{V})$ such that

$$
\mathcal{B}([p, \boldsymbol{u}],[q, \boldsymbol{v}])=\mathcal{L}([q, \boldsymbol{v}]),
$$

for all test functions $[q, \boldsymbol{v}] \in \mathrm{V}$, and satisfying the initial conditions. The bilinear form $\mathcal{B}$, the linear form $\mathcal{L}$ and the space $\mathrm{V}$ are defined in three different ways depending on the variational form into consideration. For simplicity, we will assume that the forcing terms $f_{p}$ and $\boldsymbol{f}_{u}$ are square integrable, although we could relax this regularity requirement and assume they belong to the dual space of $\mathrm{V}_{p}$ and $\mathrm{V}_{u}$, respectively. Let us also define two auxiliary variables denoted as $\kappa_{p}$ and $\kappa_{u}$ :

$$
\kappa_{p}:=\left(\frac{\mu_{p}}{\mu_{u}}\right)^{\frac{1}{2}}, \quad \kappa_{u}:=\left(\frac{\mu_{u}}{\mu_{p}}\right)^{\frac{1}{2}} .
$$

The possible variational formulations of the problem are the following [10, 11]: 
Variational Form I.

$$
\begin{aligned}
& \mathrm{V}_{p}=\left\{q \in H^{1}(\Omega) \mid q=0 \text { on } \Gamma_{p}\right\}, \\
& \mathrm{V}_{u}=\left\{\boldsymbol{v} \in H(\operatorname{div}, \Omega) \mid \gamma_{n} \boldsymbol{v}=0 \text { on } \Gamma_{u} \text { and } \gamma_{n} \boldsymbol{v} \in L^{2}\left(\Gamma_{\mathrm{o}}\right)\right\} \\
& \mathcal{B}([p, \boldsymbol{u}],[q, \boldsymbol{v}])=\mu_{p}\left(\partial_{t} p, q\right)+(\nabla \cdot \boldsymbol{u}, q)+\mu_{u}\left(\partial_{t} \boldsymbol{u}, \boldsymbol{v}\right)+(\nabla p, \boldsymbol{v}) \\
& \mathcal{L}([q, \boldsymbol{v}])=\left(f_{p}, q\right)+\left(\boldsymbol{f}_{u}, \boldsymbol{v}\right) \\
& p=0 \text { on } \Gamma_{p}, \quad \text { Strongly imposed } \\
& \gamma_{n} \boldsymbol{u}=0 \text { on } \Gamma_{u}, \quad \text { Strongly imposed } \\
& \mu_{p}^{\frac{1}{2}} p=\mu_{u}^{\frac{1}{2}} \gamma_{n} \boldsymbol{u} \text { on } \Gamma_{\mathrm{o}}, \quad \text { Strongly imposed }
\end{aligned}
$$

Variational Form II.

$$
\begin{aligned}
& \mathrm{V}_{p}=L^{2}(\Omega), \quad \mathrm{V}_{u}=\left\{\boldsymbol{v} \in H(\operatorname{div}, \Omega) \mid \gamma_{n} \boldsymbol{v}=0 \text { on } \Gamma_{u} \text { and } \gamma_{n} \boldsymbol{v} \in L^{2}\left(\Gamma_{\mathrm{o}}\right)\right\} \\
& \mathcal{B}([p, \boldsymbol{u}],[q, \boldsymbol{v}])=\mu_{p}\left(\partial_{t} p, q\right)+(\nabla \cdot \boldsymbol{u}, q)+\mu_{u}\left(\partial_{t} \boldsymbol{u}, \boldsymbol{v}\right)-(p, \nabla \cdot \boldsymbol{v})+\kappa_{u} \int_{\Gamma_{\mathrm{o}}}\left(\gamma_{n} \boldsymbol{v}\right)\left(\gamma_{n} \boldsymbol{u}\right) \mathrm{d} \Gamma \\
& \mathcal{L}([q, \boldsymbol{v}])=\left(f_{p}, q\right)+\left(\boldsymbol{f}_{u}, \boldsymbol{v}\right) \\
& p=0 \text { on } \Gamma_{p}, \quad \text { Weakly imposed } \\
& \gamma_{n} \boldsymbol{u}=0 \text { on } \Gamma_{u}, \quad \text { Strongly imposed } \\
& \mu_{p}^{\frac{1}{2}} p=\mu_{u}^{\frac{1}{2}} \gamma_{n} \boldsymbol{u} \text { on } \Gamma_{\mathrm{o}}, \quad \text { Weakly imposed }
\end{aligned}
$$

Variational Form III.

$$
\begin{aligned}
& \mathrm{V}_{p}=\left\{q \in H^{1}(\Omega) \mid q=0 \text { on } \Gamma_{p}\right\}, \quad \mathrm{V}_{u}=L^{2}(\Omega)^{d} \\
& \mathcal{B}([p, \boldsymbol{u}],[q, \boldsymbol{v}])=\mu_{p}\left(\partial_{t} p, q\right)-(\boldsymbol{u}, \nabla q)+\mu_{u}\left(\partial_{t} \boldsymbol{u}, \boldsymbol{v}\right)+(\nabla p, \boldsymbol{v})+\kappa_{p} \int_{\Gamma_{\mathrm{o}}} p q \mathrm{~d} \Gamma \\
& \mathcal{L}([q, \boldsymbol{v}])=\left(f_{p}, q\right)+\left(\boldsymbol{f}_{u}, \boldsymbol{v}\right) \\
& p=0 \text { on } \Gamma_{p}, \quad \text { Strongly imposed } \\
& \gamma_{n} \boldsymbol{u}=0 \text { on } \Gamma_{u}, \quad \text { Weakly imposed } \\
& \mu_{p}^{\frac{1}{2}} p=\mu_{u}^{\frac{1}{2}} \gamma_{n} \boldsymbol{u} \text { on } \Gamma_{\mathrm{o}}, \quad \text { Weakly imposed }
\end{aligned}
$$

\subsection{Stabilized finite element formulations}

Here we present two stabilized FE methods, which we will denote by the acronyms ASGS (Algebraic Sub-Grid Scales) and OSS (Orthogonal Sub-grid Scales), aimed at overcoming the instability problems of the standard Galerkin method found when the interpolating spaces do not satisfy an appropriate inf-sup condition. We focus on equal and continuous interpolations for $p$ and $\boldsymbol{u}$ and therefore, conforming FE spaces. For conciseness, we will consider quasi-uniform FE partitions $\{K\}$ of size $h$. For stabilized formulations in general non-uniform non-degenerate cases, see [19].

Let $\mathrm{V}_{p, h}$ and $\mathrm{V}_{u, h}$ be the FE spaces constructed from the FE partition $\{K\}$ to approximate $p$ and $\boldsymbol{u}$, respectively, with $\mathrm{V}_{p, h} \subset \mathrm{V}_{p}$ and $\mathrm{V}_{u, h} \subset \mathrm{V}_{u}$. Additionally, let us define $\mathrm{V}_{h}=\mathrm{v}_{p, h} \times \mathrm{V}_{u, h}$. Stabilized FE methods deal with the following problem: find a pair $\left[p_{h}, \boldsymbol{u}_{h}\right] \in \mathcal{C}^{1}\left(\Upsilon ; \mathfrak{V}_{h}\right)$ with initial conditions $p_{h}(\boldsymbol{x}, 0)=0$, $\boldsymbol{u}_{h}(\boldsymbol{x}, 0)=\mathbf{0}$ such that

$$
\mathcal{B}_{s}\left(\left[p_{h}, \boldsymbol{u}_{h}\right],\left[q_{h}, \boldsymbol{v}_{h}\right]\right)=\mathcal{L}_{s}\left(\left[q_{h}, \boldsymbol{v}_{h}\right]\right),
$$

for all test functions $\left[q_{h}, \boldsymbol{v}_{h}\right] \in \mathrm{V}_{h}$, where the bilinear form $\mathcal{B}_{s}$ and the linear form $\mathcal{L}_{s}$ include the Galerkin terms and additional stabilization terms. Depending on how the stabilization part is designed, a different stabilization method arises. Below, we present two methods, namely ASGS and OSS. The stabilization terms depend on the choice of the so-called stabilization parameters $\tau_{p}$ and $\tau_{u}$. 
The ASGS method is an extension to the mixed form of the wave equation of the method proposed in [20, 21]. It consists in solving problem $(20)$ and taking the bilinear form $\mathcal{B}_{s}$ and the linear form $\mathcal{L}_{s}$ as:

$$
\begin{aligned}
& \mathcal{B}_{s}\left(\left[p_{h}, \boldsymbol{u}_{h}\right],\left[q_{h}, \boldsymbol{v}_{h}\right]\right)=\mathcal{B}\left(\left[p_{h}, \boldsymbol{u}_{h}\right],\left[q_{h}, \boldsymbol{v}_{h}\right]\right)+\left(\mu_{p} \partial_{t} p_{h}+\nabla \cdot \boldsymbol{u}_{h}, \tau_{p} \nabla \cdot \boldsymbol{v}_{h}\right)+\left(\mu_{u} \partial_{t} \boldsymbol{u}_{h}+\nabla p_{h}, \tau_{u} \nabla q_{h}\right), \\
& \mathcal{L}_{s}\left(\left[q_{h}, \boldsymbol{v}_{h}\right]\right)=\mathcal{L}\left(\left[q_{h}, \boldsymbol{v}_{h}\right]\right)+\left(f_{p}, \tau_{p} \nabla \cdot \boldsymbol{v}_{h}\right)+\left(\boldsymbol{f}_{u}, \tau_{u} \nabla q_{h}\right)
\end{aligned}
$$

The OSS method is an extension to the wave equation in mixed form of the method proposed in [22, 23]. It consists in solving problem (20) and taking the bilinear form $\mathcal{B}_{s}$ and the linear form $\mathcal{L}_{s}$ as:

$$
\begin{aligned}
& \mathcal{B}_{s}\left(\left[p_{h}, \boldsymbol{u}_{h}\right],\left[q_{h}, \boldsymbol{v}_{h}\right]\right)=\mathcal{B}\left(\left[p_{h}, \boldsymbol{u}_{h}\right],\left[q_{h}, \boldsymbol{v}_{h}\right]\right)+\left(P_{p}^{\perp}\left(\nabla \cdot \boldsymbol{u}_{h}\right), \tau_{p} \nabla \cdot \boldsymbol{v}_{h}\right)+\left(P_{u}^{\perp}\left(\nabla p_{h}\right), \tau_{u} \nabla q_{h}\right), \\
& \mathcal{L}_{s}\left(\left[q_{h}, \boldsymbol{v}_{h}\right]\right)=\mathcal{L}\left(\left[q_{h}, \boldsymbol{v}_{h}\right]\right)+\left(P_{p}^{\perp}\left(f_{p}\right), \tau_{p} \nabla \cdot \boldsymbol{v}_{h}\right)+\left(P_{u}^{\perp}\left(\boldsymbol{f}_{u}\right), \tau_{u} \nabla q_{h}\right),
\end{aligned}
$$

where $P_{p}^{\perp}(\cdot)=I(\cdot)-P_{p}(\cdot)$ and $P_{u}^{\perp}(\cdot)=I(\cdot)-P_{u}(\cdot), P_{p}(\cdot)$ being the $L^{2}(\Omega)$ projection on $\mathrm{V}_{p, h}$ and $P_{u}(\cdot)$ the $L^{2}(\Omega)$ projection on $\mathrm{V}_{u, h}$. This in particular implies that $P_{p}(\cdot)=0$ on $\Gamma_{p}$ for variational forms I and III and that $\boldsymbol{n} \cdot P_{u}(\cdot)=0$ on $\Gamma_{u}$ for variational forms I and II. Let us remark that for the sake of conciseness we will not consider in this paper the so-called dynamic subscales introduced in [23], even if we favor them and their use is crucial in the case of very small time steps (see also [24, 25]).

An important ingredient of stabilized formulations are the stabilization parameters. In our case, we compute them in all formulations as:

$$
\tau_{p}=C_{\tau} h \sqrt{\frac{\mu_{u}}{\mu_{p}}} \sqrt{\frac{\ell_{p}}{\ell_{u}}}, \quad \tau_{u}=C_{\tau} h \sqrt{\frac{\mu_{p}}{\mu_{u}}} \sqrt{\frac{\ell_{u}}{\ell_{p}}},
$$

where $C_{\tau}$ is a dimensionless algorithmic constant and $\ell_{p}, \ell_{u}$ are length scales corresponding to $p$ and $\boldsymbol{u}$, respectively. As it was shown in the analysis presented in [10, in order to mimic at the discrete level the proper functional setting of the continuous problem the length scales $\ell_{p}$ and $\ell_{u}$ should be taken as shown in Table 1, where $\mathrm{L}_{0}$ is a fixed length scale of the problem that can be fixed a priori. The motivation for designing the stabilization parameters can be found in [26, 9].

Table 1: Stabilization Parameters Order and Length Scales Definition

\begin{tabular}{cccc}
\hline Variational Form & $\mathrm{I}$ & $\mathrm{II}$ & $\mathrm{III}$ \\
\hline$\ell_{p}$ & $\ell_{p}=\ell_{u}$ & $\mathrm{~L}_{0}^{2} / h$ & $h$ \\
$\ell_{u}$ & $\ell_{p}=\ell_{u}$ & $h$ & $\mathrm{~L}_{0}^{2} / h$ \\
$\tau_{p}$ & $\mathcal{O}(h)$ & $\mathcal{O}(1)$ & $\mathcal{O}\left(h^{2}\right)$ \\
$\tau_{u}$ & $\mathcal{O}(h)$ & $\mathcal{O}\left(h^{2}\right)$ & $\mathcal{O}(1)$ \\
\hline
\end{tabular}

\subsection{Full discretization}

To discretize in time we will use standard finite difference schemes of first and second order, namely, the $\mathrm{BE}$, the $\mathrm{CN}$ and the BDF2 schemes. Let $0=t^{0}<\ldots<t^{n}<\ldots t^{N}=T$ be a finite difference partition of $\Upsilon$ of size $\delta t$, that we take constant for the sake of simplicity. Let $U$ be the sequence of exact solutions $U:=\left\{U^{n}\right\}_{n=0}^{N}:=\left\{\left[p\left(\boldsymbol{x}, t^{n}\right), \boldsymbol{u}\left(\boldsymbol{x}, t^{n}\right)\right]\right\}_{n=0}^{N}$. We will often abbreviate $p^{n}:=p\left(\boldsymbol{x}, t^{n}\right)$ and $\boldsymbol{u}^{n}:=\boldsymbol{u}\left(\boldsymbol{x}, t^{n}\right) ;$ the same symbol will be used for a time approximation to these unknowns. Let $U_{h}$ be the sequence of approximate solutions of the fully discrete problem, that is $U_{h}:=\left\{U_{h}^{n}\right\}_{n=0}^{N}:=\left\{\left[p_{h}^{n}, \boldsymbol{u}_{h}^{n}\right]\right\}_{n=0}^{N}$. This fully discrete problem reads: find the sequence $U_{h}$ such that

$$
\mathcal{B}_{h}\left(U_{h}, V_{h}\right)=\mathcal{L}_{h}\left(V_{h}\right)
$$

for all $V_{h}$. The definitions of $\mathcal{B}_{h}$ and $\mathcal{L}_{h}$ depend on the combination of space and time discretization and will be given in the next section, where the stability and convergence properties of the fully discrete methods are presented. 
To simplify notation we will use the backward $\left(D_{\mathrm{B}, q, s}^{r}\right)$ central $\left(D_{\mathrm{C}, q, s}^{r}\right)$ and forward $\left(D_{\mathrm{F}, q, s}^{r}\right)$ difference operators for the $r$-th derivative, of order $q$ and in a time interval of size $s \delta t$. For instance, the $\mathrm{BE}$ and BDF2 approximations of the time derivative for the scalar unknown will be

$$
D_{\mathrm{B}, 1,1}^{1} p^{n}=\frac{p^{n}-p^{n-1}}{\delta t}, \quad D_{\mathrm{B}, 2,1}^{1} p^{n}=\frac{3 p^{n}-4 p^{n-1}+p^{n-2}}{2 \delta t} .
$$

\section{Stability and convergence results}

\subsection{Preliminaries}

We present here the results of stability and convergence of the fully discrete methods arising from the combination of time marching schemes (BE, CN and BDF2) and spatial stabilized FE methods (ASGS and OSS). We provide stability and convergence results for each fully discrete method. In order to do that, we use the concept of $\Lambda$-coercivity, used for the first time in [12. This concept is equivalent to the concept of T-coercivity [27, 28, 29, 30] but it was coined before under the name of $\Lambda$-coercivity in [12].

The concept of $\Lambda$-coercivity aids us in the proof of stability and later in the convergence analysis. The proofs are similar to the ones shown in [10, 11], but considering now the time-discretization as well. Only one of such proofs will be developed here, the others following very similar strategies.

As usual, we use $C$ for a generic constant independent of the mesh size $h$ and time step $\delta t$. The value of $C$ may be different at different occurrences. Additionally, we will use the notation $A \gtrsim B$ and $A \lesssim B$ to indicate that $A \geq C B$ and $A \leq C B$ for any $A$ and $B$ depending on the solution and the data. All our results are presented in such a way that $C$ is independent of the dimensional system, i.e., $C$ is dimensionless.

Let $U_{I}$ be the sequence of projected solutions $U_{I}:=\left\{\left[p_{I}^{n}, \boldsymbol{u}_{I}^{n}\right]\right\}_{n=0}^{N}$, where $p_{I}^{n}:=\Pi_{p}^{n}\left(p^{n}\right)$ and $\boldsymbol{u}_{I}^{n}:=$ $\Pi_{u}^{n}\left(\boldsymbol{u}^{n}\right)$, where $\Pi_{p}^{n}(\cdot)$ and $\Pi_{u}^{n}(\cdot)$ are adequate interpolants onto the FE spaces, taken to be projections. Notice that this notation allows us to take different interpolants at different time steps.

Let us define some auxiliary norms to ease notation in the following:

$$
\begin{aligned}
\left\|V^{n}\right\|_{0}^{2} & :=\mu_{p}\left\|q^{n}\right\|^{2}+\mu_{u}\left\|\boldsymbol{v}^{n}\right\|^{2}, \\
\|V\|_{B}^{2} & :=(1+\sigma) \kappa_{p}\|q\|_{\ell^{2}\left(\Upsilon, L^{2}\left(\Gamma_{\mathrm{o}}\right)\right)}^{2}+(1-\sigma) \kappa_{u}\left\|\gamma_{n} \boldsymbol{v}\right\|_{\ell^{2}\left(\Upsilon, L^{2}\left(\Gamma_{\mathrm{o}}\right)\right)}^{2}, \\
\|F\|_{F}^{2} & :=\frac{1}{\mu_{p}}\left\|f_{p}\right\|_{\ell^{1}\left(\Upsilon, L^{2}(\Omega)\right)}^{2}+\frac{1}{\mu_{u}}\left\|\boldsymbol{f}_{u}\right\|_{\ell^{1}\left(\Upsilon, L^{2}(\Omega)\right)}^{2}+\tau_{p}\left\|f_{p}\right\|_{\ell^{2}\left(\Upsilon, L^{2}(\Omega)\right)}^{2}+\tau_{u}\left\|\boldsymbol{f}_{u}\right\|_{\ell^{2}\left(\Upsilon, L^{2}(\Omega)\right)}^{2},
\end{aligned}
$$

with $\sigma=-1,0,1$ for variational forms I, II and III respectively, and $\|\cdot\|_{\ell^{p}(\Upsilon, \mathrm{x})}$ stands for the $\ell^{p}$-norm of a sequence of $\mathrm{X}$-norms associated to the time discretization of $\Upsilon$. When $\mathrm{X}=L^{q}(\Omega)$, we will use the abbreviation $\ell^{p}\left(\Upsilon, L^{q}(\Omega)\right) \equiv \ell^{p}\left(L^{q}\right)$.

For the convergence analysis we need to define three types of errors: the projection error, the discretization error, and the total error. The projection error is the error between the exact solution and the projected exact solution and is defined as $\varepsilon:=\left\{\varepsilon^{n}\right\}_{n=0}^{N}:=\left\{\left[\varepsilon_{p}^{n}, \varepsilon_{u}^{n}\right]\right\}_{n=0}^{N}:=U-U_{I}$. The discretization error is the error between the discrete solution and the projected exact solution and is defined as $e:=\left\{e^{n}\right\}_{n=0}^{N}:=\left\{\left[e_{p}^{n}, e_{u}^{n}\right]\right\}_{n=0}^{N}:=U_{h}-U_{I}$. Finally, the total error is the error between the exact solution and the discrete solution and is defined as $\xi:=\left\{\xi^{n}\right\}_{n=0}^{N}:=\left\{\left[\xi_{p}^{n}, \boldsymbol{\xi}_{u}^{n}\right]\right\}_{n=0}^{N}:=U-U_{h}$.

\subsection{Analysis strategy}

As mentioned above, all the methods considered have the form $(26)$. We have proved that all of them (ASGS and OSS for the spatial discretization, considering the three possible variational formulations, and $\mathrm{BE}, \mathrm{CN}$ and BDF2 for the time integration) are stable and optimally convergent, with the convergence order in space and time that should be expected. However, including all the proofs would be extremely long and tedious. Rather than this, we explain in what follows the analysis strategy that we have employed, give the details of the terms involved for each method in the following subsection and present then the proof of the simplest case.

For all methods we have proved $\Lambda$-coercivity, stability and convergence in an appropriate norm $\|\cdot\| \cdot \|$ that depends on the method. More precisely, we have shown that 
1. $\Lambda$-coercivity. If $V$ is a sequence of functions, either continuous or discrete in space, there exist a map $\Lambda(V)$ such that

$$
\mathcal{B}_{h}(V, \Lambda(V)) \gtrsim\|V\|^{2}, \quad\|\| \Lambda(V)\|\lesssim\| V\|\|,
$$

for all $V$. From this property one easily gets stability in the form of an inf-sup condition (see [12]).

2. Stability. The discrete solution $U_{h}$ satisfies

$$
\left\|\mid U_{h}\right\|\|\lesssim\| F \|_{*}
$$

for an appropriate norm $\|F\|_{*}$ of the data (the forcing terms).

3. Convergence. Of course, the final objective is to provide an estimate for the total error $\xi$ in some norm. We can show that if $U$ is the sequence of continuous solutions and $U_{h}$ the sequence of discrete solutions, then

$$
\left\|U-U_{h}\right\| \lesssim E(h, \delta t),
$$

for a certain error function $E(h, \delta t)$. At this point, let us remark that convergence is not a more or less straightforward consequence of stability, since the discrete problem is not consistent in the variational sense. To prove convergence we need to deal with the consistency error

$$
\mathcal{C}\left(U, V_{h}\right):=\mathcal{L}_{h}\left(V_{h}\right)-\mathcal{B}_{h}\left(U, V_{h}\right) .
$$

From this definition it is easy to arrive to

$$
\mathcal{B}_{h}\left(e, V_{h}\right)=\mathcal{B}_{h}\left(\varepsilon, V_{h}\right)+\mathcal{C}\left(U, V_{h}\right) .
$$

\subsection{Forms, norms and error functions}

Recalling that we have taken as zero the initial conditions, all methods are solely defined by the bilinear form $\mathcal{B}_{h}$ and the linear form $\mathcal{L}_{h}$. These are given for all methods in Table 2 . The only comment that needs to be made is that in practice BDF2 can be started either with BE or CN. Numerical experiments show no difference in the convergence rate, even if theoretical error estimates are not optimal for convergence in terms of $\delta t$ when BDF2 is started with BE for variational forms II and III. In other words, when BE is chosen to start BDF2, some terms of the error coming from the BE part will not be of order $\delta t^{2}$ for variational forms II or III (e.g. the term $\delta t^{2} \tau_{p}\left\|\mu_{p} \partial_{t t} p\right\|_{\ell^{2}\left(L^{2}\right)}^{2}$ for variational form II. See Tables 4 and 5 . Obviously, we are assuming $\delta t$ proportional to $h$ to exclude variational form I from this statement.

The stability analysis of the different methods relies on the expression of map $\Lambda(V)$ satisfying (30), the norm of the unknown $\|V\|$ and the norm of the forcing terms $\|F\|_{*}$. These are all given in Table 3r Note that in this table use is made of abbreviations (27)-(29). The expressions of $\|F\|_{*}$ determine the regularity required for the data in each case.

Finally, Tables 4 and 5 provide the error functions of the error estimates $(32)$ for the ASGS and the OSS methods, respectively. There are many terms in these expressions with the same convergence order. However, including all of them gives an indication of the possible sources of error, as well as of the required regularity of the continuous solution to obtain optimal convergence rates.

\subsection{A sample of the proofs: ASGS-BE method}

As an example of how to prove (30), (31) and $(32)$, we present now the proof of these results for the simplest of the six methods considered, namely, ASGS for the space discretization and BE for the time integration. The proof of the rest of methods is more or less involved, but follows the same ideas. 
Table 2: Forms that define the methods

\begin{tabular}{|c|c|}
\hline Method & $\mathcal{B}_{h}$ and $\mathcal{L}_{h}$ \\
\hline ASGS-BE & $\begin{aligned} & \mathcal{B}_{h}\left(U_{h}, V_{h}\right)= \sum_{n=1}^{N} \delta t\left[\left(\mu_{p} D_{\mathrm{B}, 1,1}^{1} p_{h}^{n}+\nabla \cdot \boldsymbol{u}_{h}^{n}, q_{h}^{n}+\tau_{p} \nabla \cdot \boldsymbol{v}_{h}^{n}\right)+\left(\mu_{u} D_{\mathrm{B}, 1,1}^{1} \boldsymbol{u}_{h}^{n}+\nabla p_{h}^{n}, \boldsymbol{v}_{h}^{n}+\tau_{u} \nabla q_{h}^{n}\right)\right] \\
& \mathcal{L}_{h}\left(V_{h}\right)=\sum_{n=1}^{N} \delta t\left(f_{p}^{n}, q_{h}^{n}+\tau_{p} \nabla \cdot \boldsymbol{v}_{h}^{n}\right)+\sum_{n=1}^{N} \delta t\left(\boldsymbol{f}_{u}^{n}, \boldsymbol{v}_{h}^{n}+\tau_{u} \nabla q_{h}^{n}\right) \\
&\end{aligned}$ \\
\hline ASGS-CN & $\begin{array}{c}\mathcal{B}_{h}\left(U_{h}, V_{h}\right)= \\
\sum_{n=1}^{N} \delta t\left[\left(\mu_{p} D_{\mathrm{B}, 1,1}^{1} p_{h}^{n}+\nabla \cdot \boldsymbol{u}_{h}^{n-\frac{1}{2}}, q_{h}^{n}+\tau_{p} \nabla \cdot \boldsymbol{v}_{h}^{n}\right)\right. \\
\left.+\left(\mu_{u} D_{\mathrm{B}, 1,1}^{1} \boldsymbol{u}_{h}^{n}+\nabla p_{h}^{n-\frac{1}{2}}, \boldsymbol{v}_{h}^{n}+\tau_{u} \nabla q_{h}^{n}\right)\right] \\
\mathcal{L}_{h}\left(V_{h}\right)=\sum_{n=1}^{N} \delta t\left(f_{p}{ }^{n-\frac{1}{2}}, q_{h}^{n}+\tau_{p} \nabla \cdot \boldsymbol{v}_{h}^{n}\right)+\sum_{n=1}^{N} \delta t\left(\boldsymbol{f}_{u}{ }^{n-\frac{1}{2}}, \boldsymbol{v}_{h}^{n}+\tau_{u} \nabla q_{h}^{n}\right)\end{array}$ \\
\hline ASGS-BDF2 & $\begin{aligned} & \mathcal{B}_{h}\left(U_{h}, V_{h}\right)=\delta t {\left[\left(\mu_{p} D_{\mathrm{B}, 1,1}^{1} p_{h}^{1}+\nabla \cdot \boldsymbol{u}_{h}^{1-\frac{1}{2}}, q_{h}^{1}+\tau_{p} \nabla \cdot \boldsymbol{v}_{h}^{1}\right)+\left(\mu_{u} D_{\mathrm{B}, 1,1}^{1} \boldsymbol{u}_{h}^{1}+\nabla p_{h}^{1-\frac{1}{2}}, \boldsymbol{v}_{h}^{1}+\tau_{u} \nabla q_{h}^{1}\right)\right] } \\
&+\sum_{n=2}^{N} \delta t {\left[\left(\mu_{p} D_{\mathrm{B}, 2,1}^{1} p_{h}^{n}+\nabla \cdot \boldsymbol{u}_{h}^{n}, q_{h}^{n}+\tau_{p} \nabla \cdot \boldsymbol{v}_{h}^{n}\right)+\left(\mu_{u} D_{\mathrm{B}, 2,1}^{1} \boldsymbol{u}_{h}^{n}+\nabla p_{h}^{n}, \boldsymbol{v}_{h}^{n}+\tau_{u} \nabla q_{h}^{n}\right)\right] } \\
& \mathcal{L}_{h}\left(V_{h}\right)=\delta t\left(f_{p}{ }^{1-\frac{1}{2}}, q_{h}^{1}+\tau_{p} \nabla \cdot \boldsymbol{v}_{h}^{1}\right)+\sum_{n=2}^{N} \delta t\left(f_{p}{ }^{n}, q_{h}^{n}+\tau_{p} \nabla \cdot \boldsymbol{v}_{h}^{n}\right) \\
&+\delta t\left(\boldsymbol{f}_{u}{ }^{1-\frac{1}{2}}, \boldsymbol{v}_{h}^{1}+\tau_{u} \nabla q_{h}^{1}\right)+\sum_{n=2}^{N} \delta t\left(\boldsymbol{f}_{u}{ }^{n}, \boldsymbol{v}_{h}^{n}+\tau_{u} \nabla q_{h}^{n}\right)\end{aligned}$ \\
\hline OSS-BE & $\begin{array}{c}\mathcal{B}_{h}\left(U_{h}, V_{h}\right)=\sum_{n=1}^{N} \delta t\left[\left(\mu_{p} D_{\mathrm{B}, 1,1}^{1} p_{h}^{n}+\nabla \cdot \boldsymbol{u}_{h}^{n}, q_{h}^{n}\right)+\tau_{p}\left(P_{p}^{\perp}\left(\nabla \cdot \boldsymbol{u}_{h}^{n}\right), \nabla \cdot \boldsymbol{v}_{h}^{n}\right)\right] \\
+\sum_{n=1}^{N} \delta t\left[\left(\mu_{u} D_{\mathrm{B}, 1,1}^{1} \boldsymbol{u}_{h}^{n}+\nabla p_{h}^{n}, \boldsymbol{v}_{h}^{n}\right)+\tau_{u}\left(P_{u}^{\perp}\left(\nabla p_{h}^{n}\right), \nabla q_{h}^{n}\right)\right] \\
\mathcal{L}_{h}\left(V_{h}\right)=\sum_{n=1}^{N} \delta t\left[\left(f_{p}^{n}, q_{h}^{n}\right)+\tau_{p}\left(P_{p}^{\perp}\left(f_{p}^{n}\right), \nabla \cdot \boldsymbol{v}_{h}^{n}\right)\right]+\sum_{n=1}^{N} \delta t\left[\left(\boldsymbol{f}_{u}^{n}, \boldsymbol{v}_{h}^{n}\right)+\tau_{u}\left(P_{u}^{\perp}\left(\boldsymbol{f}_{u}{ }^{n}\right), \nabla q_{h}^{n}\right)\right]\end{array}$ \\
\hline OSS-CN & $\begin{array}{c}\mathcal{B}_{h}\left(U_{h}, V_{h}\right)=\sum_{n=1}^{N} \delta t\left[\left(\mu_{p} D_{\mathrm{B}, 1,1}^{1} p_{h}^{n}+\nabla \cdot \boldsymbol{u}_{h}^{n-\frac{1}{2}}, q_{h}^{n}\right)+\tau_{p}\left(P_{p}^{\perp}\left(\nabla \cdot \boldsymbol{u}_{h}^{n-\frac{1}{2}}\right), \nabla \cdot \boldsymbol{v}_{h}^{n}\right)\right] \\
+\sum_{n=1}^{N} \delta t\left[\left(\mu_{u} D_{\mathrm{B}, 1,1}^{1} \boldsymbol{u}_{h}^{n}+\nabla p_{h}^{n-\frac{1}{2}}, \boldsymbol{v}_{h}^{n}\right)+\tau_{u}\left(P_{u}^{\perp}\left(\nabla p_{h}^{n-\frac{1}{2}}\right), \nabla q_{h}^{n}\right)\right] \\
\mathcal{L}_{h}\left(V_{h}\right)=\sum_{n=1}^{N} \delta t\left[\left(f_{p}{ }^{n-\frac{1}{2}}, q_{h}^{n}\right)+\tau_{p}\left(P_{p}^{\perp}\left(f_{p}{ }^{n-\frac{1}{2}}\right), \nabla \cdot \boldsymbol{v}_{h}^{n}\right)\right] \\
+\sum_{n=1}^{N} \delta t\left[\left(\boldsymbol{f}_{u}{ }^{n-\frac{1}{2}}, \boldsymbol{v}_{h}^{n}\right)+\tau_{u}\left(P_{u}^{\perp}\left(\boldsymbol{f}_{u}{ }^{n-\frac{1}{2}}\right), \nabla q_{h}^{n}\right)\right]\end{array}$ \\
\hline OSS-BDF2 & $\begin{array}{c}\mathcal{B}_{h}\left(U_{h}, V_{h}\right)=\delta t\left[\left(\mu_{p} D_{\mathrm{B}, 1,1}^{1} p_{h}^{1}+\nabla \cdot \boldsymbol{u}_{h}^{\frac{1}{2}}, q_{h}^{1}+\tau_{p} \nabla \cdot \boldsymbol{v}_{h}^{1}\right)+\left(P_{p}^{\perp}\left(\nabla \cdot \boldsymbol{u}_{h}^{\frac{1}{2}}\right), \tau_{p} \nabla \cdot \boldsymbol{v}_{h}^{1}\right)\right] \\
+\sum_{n=2}^{N} \delta t\left[\left(\mu_{p} D_{\mathrm{B}, 2,1}^{1} p_{h}^{n}+\nabla \cdot \boldsymbol{u}_{h}^{n}, q_{h}^{n}\right)+\tau_{p}\left(P_{p}^{\perp}\left(\nabla \cdot \boldsymbol{u}_{h}^{n}\right), \nabla \cdot \boldsymbol{v}_{h}^{n}\right)\right] \\
+\delta t\left[\left(\mu_{u} D_{\mathrm{B}, 1,1}^{1} \boldsymbol{u}_{h}^{1}+\nabla p_{h}^{\frac{1}{2}}, \boldsymbol{v}_{h}^{1}\right)+\left(P_{u}^{\perp}\left(\nabla p_{h}^{\frac{1}{2}}\right), \tau_{u} \nabla q_{h}^{1}\right)\right] \\
+\sum_{n=2}^{N} \delta t\left[\left(\mu_{u} D_{\mathrm{B}, 2,1}^{1} \boldsymbol{u}_{h}^{n}+\nabla p_{h}^{n}, \boldsymbol{v}_{h}^{n}\right)+\tau_{u}\left(P_{u}^{\perp}\left(\nabla p_{h}^{n}\right), \nabla q_{h}^{n}\right)\right] \\
\mathcal{L}_{h}\left(V_{h}\right)=\delta t\left[\left(f_{p}^{\frac{1}{2}}, q_{h}^{1}\right)+\tau_{p}\left(P_{p}^{\perp}\left(f_{p}^{\frac{1}{2}}\right), \nabla \cdot \boldsymbol{v}_{h}^{1}\right)\right]+\delta t\left[\left(\boldsymbol{f}_{u}^{\frac{1}{2}}, \boldsymbol{v}_{h}^{1}\right)+\tau_{u}\left(P_{u}^{\perp}\left(\boldsymbol{f}_{u}^{\frac{1}{2}}\right), \nabla q_{h}^{1}\right)\right] \\
+\sum_{n=2}^{N} \delta t\left[\left(f_{p}^{n}, q_{h}^{n}\right)+\tau_{p}\left(P_{p}^{\perp}\left(f_{p}^{n}\right), \nabla \cdot \boldsymbol{v}_{h}^{n}\right)\right]+\sum_{n=2}^{N} \delta t\left[\left(\boldsymbol{f}_{u}^{n}, \boldsymbol{v}_{h}^{n}\right)+\tau_{u}\left(P_{u}^{\perp}\left(\boldsymbol{f}_{u}^{n}\right), \nabla q_{h}^{n}\right)\right]\end{array}$ \\
\hline
\end{tabular}

\subsubsection{Proof of $\Lambda$-coercivity 30}

From the definition of $\mathcal{B}_{h}$ in Table 2 and of $\Lambda(V)$ in Table 3 for the ASGS-BE method we get

$$
\begin{aligned}
\mathcal{B}_{h}(V, \Lambda(V))= & \sum_{n=1}^{N} \delta t\left(\mu_{p} D_{\mathrm{B}, 1,1}^{1} q^{n}, q^{n}\right)+\sum_{n=1}^{N} \delta t\left(\mu_{u} D_{\mathrm{B}, 1,1}^{1} \boldsymbol{v}^{n}, \boldsymbol{v}^{n}\right) \\
& +\tau_{p} \tau_{u} \mu_{u} \sum_{n=1}^{N} \delta t\left(\nabla \cdot \boldsymbol{v}^{n}, \nabla \cdot\left(D_{\mathrm{B}, 1,1}^{1} \boldsymbol{v}^{n}\right)\right)+\tau_{p} \tau_{u} \mu_{p} \sum_{n=1}^{N} \delta t\left(\nabla q^{n}, \nabla\left(D_{\mathrm{B}, 1,1}^{1} q^{n}\right)\right) \\
& +\sum_{n=1}^{N} \delta t\left[\left(\nabla \cdot \boldsymbol{v}^{n}, q^{n}\right)+\left(\nabla q^{n}, \boldsymbol{v}^{n}\right)\right] \\
& +\sum_{n=1}^{N} \delta t \tau_{p}\left\|\mu_{p} D_{\mathrm{B}, 1,1}^{1} q^{n}+\nabla \cdot \boldsymbol{v}^{n}\right\|^{2}+\sum_{n=1}^{N} \delta t \tau_{u}\left\|\mu_{u} D_{\mathrm{B}, 1,1}^{1} \boldsymbol{v}^{n}+\nabla q^{n}\right\|^{2} \\
& +\tau_{p} \tau_{u} \mu_{p} \mu_{u} \sum_{n=1}^{N} \delta t\left[\left(D_{\mathrm{B}, 1,1}^{1} q^{n}, \nabla \cdot\left(D_{\mathrm{B}, 1,1}^{1} \boldsymbol{v}^{n}\right)\right)+\left(D_{\mathrm{B}, 1,1}^{1} \boldsymbol{v}^{n}, \nabla\left(D_{\mathrm{B}, 1,1}^{1} q^{n}\right)\right)\right]
\end{aligned}
$$


Table 3: Functions to obtain $\Lambda$-coercivity and norms

\begin{tabular}{|c|c|}
\hline Method & $\Lambda(V),\|\| V\|\|$ and $\|F\|_{*}$ \\
\hline ASGS-BE & $\begin{array}{c}\Lambda(V):=V+\left\{[0, \mathbf{0}],\left\{\left[\tau_{p} \mu_{p} D_{\mathrm{B}, 1,1}^{1} q^{n}, \tau_{u} \mu_{u} D_{\mathrm{B}, 1,1}^{1} \boldsymbol{v}^{n}\right]\right\}_{n=1}^{N}\right\} \\
\|V\|\left\|^{2}:=\right\| V^{N}\left\|_{0}^{2}+\right\| V\left\|_{B}^{2}+\tau_{p}\right\| \mu_{p} D_{\mathrm{B}, 1,1}^{1} q^{n}+\nabla \cdot \boldsymbol{v}^{n}\left\|_{\ell^{2}\left(\Upsilon, L^{2}(\Omega)\right)}^{2}+\tau_{u}\right\| \mu_{u} D_{\mathrm{B}, 1,1}^{1} \boldsymbol{v}^{n}+\nabla q^{n} \|_{\ell^{2}\left(\Upsilon, L^{2}(\Omega)\right)}^{2} \\
\|F\|_{*}{ }^{2}:=\|F\|_{F}^{2}+\tau_{p} \tau_{u} \mu_{u}\left\|D_{\mathrm{B}, 1,1}^{1} f_{p}\right\|_{\ell^{1}\left(\Upsilon, L^{2}(\Omega)\right)}^{2}+\tau_{p} \tau_{u} \mu_{p}\left\|D_{\mathrm{B}, 1,1}^{1} \boldsymbol{f}_{u}\right\|_{\ell^{1}\left(\Upsilon, L^{2}(\Omega)\right)}^{2} \\
+\tau_{p} \tau_{u} \mu_{u}\left\|f_{p}\right\|_{\ell^{\infty}\left(\Upsilon, L^{2}(\Omega)\right)}^{2}+\tau_{p} \tau_{u} \mu_{p}\left\|\boldsymbol{f}_{u}\right\|_{\ell \infty\left(\Upsilon, L^{2}(\Omega)\right)}^{2}\end{array}$ \\
\hline ASGS-CN & $\begin{array}{c}\Lambda(V):=\left\{\left[p^{0}, \boldsymbol{u}^{0}\right],\left\{\left[p^{n-\frac{1}{2}}, \boldsymbol{u}^{n-\frac{1}{2}}\right]\right\}_{n=1}^{N}\right\}+\left\{[0, \mathbf{0}],\left\{\left[\tau_{p} \mu_{p} D_{\mathrm{B}, 1,1}^{1} q^{n}, \tau_{u} \mu_{u} D_{\mathrm{B}, 1,1}^{1} \boldsymbol{v}^{n}\right]\right\}_{n=1}^{N}\right\} \\
\|V\|^{2}:=\left\|V^{N}\right\|_{0}^{2}+\|V\|_{B}^{2}+\tau_{p}\left\|\mu_{p} D_{\mathrm{B}, 1,1}^{1} q^{n}+\nabla \cdot \boldsymbol{v}^{n-\frac{1}{2}}\right\|_{\ell^{2}\left(\Upsilon, L^{2}(\Omega)\right)}^{2} \\
+\tau_{u}\left\|\mu_{u} D_{\mathrm{B}, 1,1}^{1} \boldsymbol{v}^{n}+\nabla q^{n-\frac{1}{2}}\right\|_{\ell^{2}\left(\Upsilon, L^{2}(\Omega)\right)}^{2} \\
\|\|_{*}^{2}:=\|F\|_{F}^{2}+\tau_{p} \tau_{u} \mu_{u}\left\|D_{\mathrm{B}, 1,1}^{1} f_{p}\right\|_{\ell^{1}\left(\Upsilon, L^{2}(\Omega)\right)}^{2}+\tau_{p} \tau_{u} \mu_{p}\left\|D_{\mathrm{B}, 1,1}^{1} \boldsymbol{f}_{u}\right\|_{\ell^{1}\left(\Upsilon, L^{2}(\Omega)\right)}^{2} \\
+\tau_{p} \tau_{u} \mu_{u}\left\|f_{p}\right\|_{\ell^{\infty}\left(\Upsilon, L^{2}(\Omega)\right)}^{2}+\tau_{p} \tau_{u} \mu_{p}\left\|\boldsymbol{f}_{u}\right\|_{\ell^{\infty}\left(\Upsilon, L^{2}(\Omega)\right)}^{2}\end{array}$ \\
\hline ASGS-BDF2 & $\begin{array}{c}\Lambda(V):=\left\{V^{0}, V^{\frac{1}{2}},\left\{V^{n}\right\}_{n=2}^{N}\right\} \\
+\left\{[0, \mathbf{0}],\left[\tau_{p} \mu_{p} D_{\mathrm{B}, 1,1}^{1} q^{1}, \tau_{u} \mu_{u} D_{\mathrm{B}, 1,1}^{1} \boldsymbol{v}^{1}\right],\left\{\left[\tau_{p} \mu_{p} D_{\mathrm{B}, 2,1}^{1} q^{n}, \tau_{u} \mu_{u} D_{\mathrm{B}, 2,1}^{1} \boldsymbol{v}^{n}\right]\right\}_{n=2}^{N}\right\} \\
\|V\|^{2}:=\left\|V^{N}\right\|_{0}^{2}+\|V\|_{B}^{2}+\tau_{p}\left\|\mu_{p} D_{\mathrm{B}, 1,1}^{1} q^{1}+\nabla \cdot \boldsymbol{v}^{\frac{1}{2}}\right\|^{2} \delta t+\tau_{u}\left\|\mu_{u} D_{\mathrm{B}, 1,1}^{1} \boldsymbol{v}^{1}+\nabla q^{\frac{1}{2}}\right\|^{2} \delta t \\
+\tau_{p}\left\|\mu_{p} D_{\mathrm{B}, 2,1}^{1} q^{n}+\nabla \cdot \boldsymbol{v}^{n}\right\|_{\ell^{2}\left(\Upsilon, L^{2}(\Omega)\right)}^{2}+\tau_{u}\left\|\mu_{u} D_{\mathrm{B}, 2,1}^{1} \boldsymbol{v}^{n}+\nabla q^{n}\right\|_{\ell^{2}\left(\Upsilon, L^{2}(\Omega)\right)}^{2} \\
\|F\|_{*}^{2}:=\|F\|_{F}^{2}+\tau_{p} \tau_{u} \mu_{u}\left\|D_{\mathrm{B}, 1,1}^{1} f_{p}{ }^{1}\right\|^{2} \delta t^{2}+\tau_{p} \tau_{u} \mu_{u}\left\|D_{\mathrm{B}, 2,1}^{1} f_{p}\right\|_{\ell^{1}\left(\Upsilon, L^{2}(\Omega)\right)}^{2}+\tau_{p} \tau_{u} \mu_{u}\left\|f_{p}\right\|_{\ell^{\infty}\left(\Upsilon, L^{2}(\Omega)\right)}^{2} \\
+\tau_{p} \tau_{u} \mu_{p}\left\|D_{\mathrm{B}, 1,1}^{1} \boldsymbol{f}_{u}{ }^{1}\right\|^{2} \delta t^{2}+\tau_{p} \tau_{u} \mu_{p}\left\|D_{\mathrm{B}, 2,1}^{1} \boldsymbol{f}_{u}\right\|_{\ell^{1}\left(\Upsilon, L^{2}(\Omega)\right)}^{2}+\tau_{p} \tau_{u} \mu_{p}\left\|\boldsymbol{f}_{u}\right\|_{\ell \infty}^{2}\left(\Upsilon, L^{2}(\Omega)\right)\end{array}$ \\
\hline OSS-BE & $\begin{array}{c}\Lambda(V):=V+\beta \Lambda_{b}(V) \quad(\beta \text { small enough }) \\
\Lambda_{b}(V):=\left\{[0, \mathbf{0}],\left\{\left[\tau_{p}\left(\mu_{p} D_{\mathrm{B}, 1,1}^{1} q^{n}+P_{p}\left(\nabla \cdot \boldsymbol{v}^{n}\right)\right), \tau_{u}\left(\mu_{u} D_{\mathrm{B}, 1,1}^{1} \boldsymbol{v}^{n}+P_{u}\left(\nabla q^{n}\right)\right)\right]\right\}_{n \overline{\bar{N}}_{1}}^{N}\right\} \\
\|V\|^{2}:=\left\|V^{N}\right\|_{0}^{2}+\|V\|_{B}^{2}+\tau_{p}\left\|\mu_{p} D_{\mathrm{B}, 1,1}^{1} q^{n}+\nabla \cdot \boldsymbol{v}^{n}\right\|_{\ell^{2}\left(\Upsilon, L^{2}(\Omega)\right)}^{2}+\tau_{u}\left\|\mu_{u} D_{\mathrm{B}, 1,1}^{1} \boldsymbol{v}^{n}+\nabla q^{n}\right\|_{\ell^{2}\left(\Upsilon, L^{2}(\Omega)\right)} \\
\|F\|_{*}^{2}:=\|F\|_{F}^{2}\end{array}$ \\
\hline OSS-CN & $\begin{array}{c}\Lambda(V):=\Lambda_{a}(V)+\beta \Lambda_{b}(V)(\beta \text { small enough }) \\
\Lambda_{a}(V):=\left\{\left[p^{0}, \boldsymbol{u}^{0}\right],\left\{\left[p^{n-\frac{1}{2}}, \boldsymbol{u}^{n-\frac{1}{2}}\right]\right\}_{n=1}^{N}\right\} \\
\Lambda_{b}(V):=\left\{[0, \mathbf{0}],\left\{\left[\tau_{p}\left(\mu_{p} D_{\mathrm{B}, 1,1}^{1} q^{n}+P_{p}\left(\nabla \cdot \boldsymbol{v}^{n-\frac{1}{2}}\right)\right), \tau_{u}\left(\mu_{u} D_{\mathrm{B}, 1,1}^{1} \boldsymbol{v}^{n}+P_{u}\left(\nabla q^{n-\frac{1}{2}}\right)\right)\right]\right\}_{n=1}^{N}\right\} \\
\|V\|^{2}:=\left\|V^{N}\right\|_{0}^{2}+\|V\|_{B}^{2}+\tau_{p}\left\|\mu_{p} D_{\mathrm{B}, 1,1}^{1} q^{n}+\nabla \cdot \boldsymbol{v}^{n-\frac{1}{2}}\right\|_{\ell^{2}\left(\Upsilon, L^{2}(\Omega)\right)}^{2} \\
+\tau_{u}\left\|\mu_{u} D_{\mathrm{B}, 1,1}^{1} \boldsymbol{v}^{n}+\nabla q^{n-\frac{1}{2}}\right\|_{\ell^{2}\left(\Upsilon, L^{2}(\Omega)\right)}^{2} \\
\|F\|_{*}^{2}:=\|F\|_{F}^{2}\end{array}$ \\
\hline OSS-BDF2 & $\begin{array}{c}\Lambda(V):=\Lambda_{a}(V)+\beta \Lambda_{b}(V)(\beta>0 \text { small enough }) \\
\Lambda_{a}(V):=\left\{V^{0}, V^{\frac{1}{2}},\left\{V^{n}\right\}_{n=2}^{N}\right\} \\
\Lambda_{b}(V):=\left\{[0, \mathbf{0}],\left[\tau_{p}\left(\mu_{p} D_{\mathrm{B}, 1,1}^{1} q^{1}+P_{p}\left(\nabla \cdot \boldsymbol{v}^{\frac{1}{2}}\right)\right), \tau_{u}\left(\mu_{u} D_{\mathrm{B}, 1,1}^{1} \boldsymbol{v}^{1}+P_{u}\left(\nabla q^{\frac{1}{2}}\right)\right)\right]\right. \\
\left.\left\{\left[\tau_{p}\left(\mu_{p} D_{\mathrm{B}, 2,1}^{1} q^{n}+P_{p}\left(\nabla \cdot \boldsymbol{v}^{n}\right)\right), \tau_{u}\left(\mu_{u} D_{\mathrm{B}, 2,1}^{1} \boldsymbol{v}^{n}+P_{u}\left(\nabla q^{n}\right)\right)\right]\right\}_{n=2}^{N}\right\} \\
\|V\|^{2}:=\|\| V^{N}\left\|_{0}^{2}+\right\| V\left\|_{B}^{2}+\tau_{p}\right\| \mu_{p} D_{\mathrm{B}, 1,1}^{1} q^{1}+\nabla \cdot \boldsymbol{v}^{\frac{1}{2}}\left\|^{2} \delta t+\tau_{u}\right\| \mu_{u} D_{\mathrm{B}, 1,1}^{1} \boldsymbol{v}^{1}+\nabla q^{\frac{1}{2}} \|^{2} \delta t \\
\left.+\tau_{p}\left\|\mu_{p} D_{\mathrm{B}, 2,1}^{1} q^{n}+\nabla \cdot \boldsymbol{v}^{n}\right\|_{\ell^{2}\left(\Upsilon, L^{2}(\Omega)\right)}^{2}+\tau_{u}\left\|\mu_{u} D_{\mathrm{B}, 2,1}^{1} \boldsymbol{v}^{n}+\nabla q^{n}\right\|_{\ell^{2}\left(\Upsilon, L^{2}(\Omega)\right)}^{2}\right) \\
\|F\|_{*}^{2}:=\|F\|_{F}^{2}\end{array}$ \\
\hline
\end{tabular}

Now we show how each term is bounded. The first four terms of 35 can be bounded considering zero initial conditions (3), the fact that $\tau_{p} \tau_{u}=C_{\tau}^{2} h^{2}$ by 25 and the definition of $\left.\left\|V^{N}\right\|\right|_{0} ^{2}$ in (27) as

$$
\sum_{n=1}^{N} \delta t\left(\mu_{p} D_{\mathrm{B}, 1,1}^{1} q^{n}, q^{n}\right)+\sum_{n=1}^{N} \delta t\left(\mu_{u} D_{\mathrm{B}, 1,1}^{1} \boldsymbol{v}^{n}, \boldsymbol{v}^{n}\right)
$$


Table 4: Error functions I: ASGS formulation

\begin{tabular}{|c|c|}
\hline Method & $E(h, \delta t)$ \\
\hline $\mathrm{BE}$ & $\begin{array}{l}E^{2}(h, \delta t):=\mu_{p}\left\|\varepsilon_{p}\right\|_{\ell^{\infty}\left(L^{2}\right)}^{2}+\mu_{u}\left\|\varepsilon_{u}\right\|_{\ell^{\infty}\left(L^{2}\right)}^{2}+\tau_{p} \tau_{u} \mu_{u}\left\|\nabla \cdot \varepsilon_{u}\right\|_{\ell^{\infty}\left(L^{2}\right)}^{2}+\tau_{p} \tau_{u} \mu_{p}\left\|\nabla \varepsilon_{p}\right\|_{\ell^{\infty}\left(L^{2}\right)}^{2} \\
\quad+\frac{1}{\tau_{u}}\left\|\varepsilon_{u}\right\|_{\ell^{2}\left(L^{2}\right)}^{2}+\tau_{p}\left\|\mu_{p} D_{\mathrm{B}, 1,1}^{1} \varepsilon_{p}\right\|_{\ell^{2}\left(L^{2}\right)}^{2}+\tau_{p}\left\|\nabla \cdot \varepsilon_{u}\right\|_{\ell^{2}\left(L^{2}\right)}^{2}+(1+\sigma) \kappa_{p}\left\|\varepsilon_{p}\right\|_{\ell^{2}\left(\Upsilon, L^{2}\left(\Gamma_{\mathrm{o}}\right)\right)}^{2} \\
\quad+\frac{1}{\tau_{p}}\left\|\varepsilon_{p}\right\|_{\ell^{2}\left(L^{2}\right)}^{2}+\tau_{u}\left\|\mu_{u} D_{\mathrm{B}, 1,1}^{1} \varepsilon_{u}\right\|_{\ell^{2}\left(L^{2}\right)}^{2}+\tau_{u}\left\|\nabla \varepsilon_{p}\right\|_{\ell^{2}\left(L^{2}\right)}^{2}+(1-\sigma) \kappa_{u}\left\|\varepsilon_{u}\right\|_{\ell^{2}\left(\Upsilon, L^{2}\left(\Gamma_{\mathrm{o}}\right)\right)}^{2} \\
+\tau_{p} \tau_{u} \mu_{u}\left\|\mu_{p} D_{\mathrm{B}, 1,1}^{1} \varepsilon_{p}\right\|_{\ell^{\infty}\left(L^{2}\right)}^{2}+\tau_{p} \tau_{u} \mu_{u}\left\|\mu_{p} D_{\mathrm{C}, 2,1}^{2} \varepsilon_{p}\right\|_{\ell^{1}\left(L^{2}\right)}^{2}+\tau_{p} \tau_{u} \mu_{u}\left\|\nabla \cdot D_{\mathrm{B}, 1,1}^{1} \varepsilon_{u}\right\|_{\ell^{1}\left(L^{2}\right)}^{2} \\
+\tau_{p} \tau_{u} \mu_{p}\left\|\mu_{u} D_{\mathrm{B}, 1,1}^{1} \varepsilon_{u}\right\|_{\ell^{\infty}\left(L^{2}\right)}^{2}+\tau_{p} \tau_{u} \mu_{p}\left\|\mu_{u} D_{\mathrm{C}, 2,1}^{2} \varepsilon_{u}\right\|_{\ell^{1}\left(L^{2}\right)}^{2}+\tau_{p} \tau_{u} \mu_{p}\left\|\nabla D_{\mathrm{B}, 1,1}^{1} \varepsilon_{p}\right\|_{\ell^{1}\left(L^{2}\right)}^{2} \\
+\frac{\delta t^{2}}{\mu_{p}}\left\|\mu_{p} \partial_{t t} p\right\|_{\ell^{1}\left(L^{2}\right)}^{2}+\delta t^{2} \tau_{p}\left\|\mu_{p} \partial_{t t} p\right\|_{\ell^{2}\left(L^{2}\right)}^{2}+\tau_{p} \tau_{u} \mu_{u} \delta t^{2}\left(\left\|\mu_{p} \partial_{t t} p\right\|_{\ell^{\infty}\left(L^{2}\right)}^{2}+\left\|\mu_{p} \partial_{t t t} p\right\|_{\ell^{1}\left(L^{2}\right)}^{2}\right) \\
+\frac{\delta t^{2}}{\mu_{u}}\left\|\mu_{u} \partial_{t t} \boldsymbol{u}\right\|_{\ell^{1}\left(L^{2}\right)}^{2}+\delta t^{2} \tau_{u}\left\|\mu_{u} \partial_{t t} \boldsymbol{u}\right\|_{\ell^{2}\left(L^{2}\right)}^{2}+\tau_{p} \tau_{u} \mu_{p} \delta t^{2}\left(\left\|\mu_{u} \partial_{t t} \boldsymbol{u}\right\|_{\ell^{\infty}\left(L^{2}\right)}^{2}+\left\|\mu_{u} \partial_{t t t} \boldsymbol{u}\right\|_{\ell^{1}\left(L^{2}\right)}^{2}\right)\end{array}$ \\
\hline $\mathrm{CN}$ & $\begin{aligned} & E^{2}(h, \delta t):= \mu_{p}\left\|\varepsilon_{p}\right\|_{\ell^{\infty}\left(\Upsilon, L^{2}(\Omega)\right)}^{2}+\mu_{u}\left\|\varepsilon_{u}\right\|_{\ell^{\infty}\left(\Upsilon, L^{2}(\Omega)\right)}^{2}+(1+\sigma) \kappa_{p}\left\|\varepsilon_{p}\right\|_{\ell^{2}\left(\Upsilon, L^{2}\left(\Gamma_{\mathrm{o}}\right)\right)}^{2}+(1-\sigma) \kappa_{u}\left\|\varepsilon_{u}\right\|_{\ell^{2}\left(\Upsilon, L^{2}\left(\Gamma_{\mathrm{o}}\right)\right)}^{2} \\
&+ \frac{1}{\tau_{u}}\left\|\varepsilon_{u}\right\|_{\ell^{2}\left(L^{2}\right)}^{2}+\tau_{p}\left\|\mu_{p} D_{\mathrm{B}, 1,1}^{1} \varepsilon_{p}\right\|_{\ell^{2}\left(L^{2}\right)}^{2}+\tau_{p}\left\|\nabla \cdot \varepsilon_{u}\right\|_{\ell^{2}\left(L^{2}\right)}^{2}+\tau_{p} \tau_{u} \mu_{u}\left\|\nabla \cdot D_{\mathrm{B}, 1,1}^{1} \varepsilon_{u}\right\|_{\ell^{1}\left(L^{2}\right)}^{2} \\
&+\frac{1}{\tau_{p}}\left\|\varepsilon_{p}\right\|_{\ell^{2}\left(L^{2}\right)}^{2}+\tau_{u}\left\|\mu_{u} D_{\mathrm{B}, 1,1}^{1} \varepsilon_{u}\right\|_{\ell^{2}\left(L^{2}\right)}^{2}+\tau_{u}\left\|\nabla \varepsilon_{p}\right\|_{\ell^{2}\left(L^{2}\right)}^{2}+\tau_{p} \tau_{u} \mu_{p}\left\|\nabla D_{\mathrm{B}, 1,1}^{1} \varepsilon_{p}\right\|_{\ell^{1}\left(L^{2}\right)}^{2} \\
&+\tau_{p} \tau_{u} \mu_{u}\left\|\mu_{p} D_{\mathrm{B}, 1,1}^{1} \varepsilon_{p}\right\|_{\ell^{\infty}\left(L^{2}\right)}^{2}+\tau_{p} \tau_{u} \mu_{u}\left\|\mu_{p} D_{\mathrm{C}, 2,1}^{2} \varepsilon_{p}\right\|_{\ell^{1}\left(L^{2}\right)}^{2} \\
&+\tau_{p} \tau_{u} \mu_{p}\left\|\mu_{u} D_{\mathrm{B}, 1,1}^{1} \varepsilon_{u}\right\|_{\ell^{\infty}\left(L^{2}\right)}^{2}+\tau_{p} \tau_{u} \mu_{p}\left\|\mu_{u} D_{\mathrm{C}, 2,1}^{2} \varepsilon_{u}\right\|_{\ell^{1}\left(L^{2}\right)}^{2} \\
&+\frac{\delta t^{4}}{\mu_{p}}\left\|\mu_{p} \partial_{t t t} p\right\|_{\ell^{1}\left(L^{2}\right)}^{2}+\delta t^{4} \tau_{p}\left\|\mu_{p} \partial_{t t t} p\right\|_{\ell^{2}\left(L^{2}\right)}^{2}+\tau_{p} \tau_{u} \mu_{u} \delta t^{4}\left[\left\|\mu_{p} \partial_{t t t} p\right\|_{\ell^{\infty}\left(L^{2}\right)}^{2}+\left\|\mu_{p} \partial_{t t t t} p\right\|_{\ell^{1}\left(L^{2}\right)}^{2}\right] \\
&+\frac{\delta t^{4}}{\mu_{u}}\left\|\mu_{u} \partial_{t t t} \boldsymbol{u}\right\|_{\ell^{1}\left(L^{2}\right)}^{2}+\delta t^{4} \tau_{u}\left\|\mu_{u} \partial_{t t t} \boldsymbol{u}\right\|_{\ell^{2}\left(L^{2}\right)}^{2}+\tau_{p} \tau_{u} \mu_{p} \delta t^{4}\left[\left\|\mu_{u} \partial_{t t t} \boldsymbol{u}\right\|_{\ell^{\infty}\left(L^{2}\right)}^{2}+\left\|\mu_{u} \partial_{t t t t} \boldsymbol{u}\right\|_{\ell^{1}\left(L^{2}\right)}^{2}\right]\end{aligned}$ \\
\hline BDF2 & 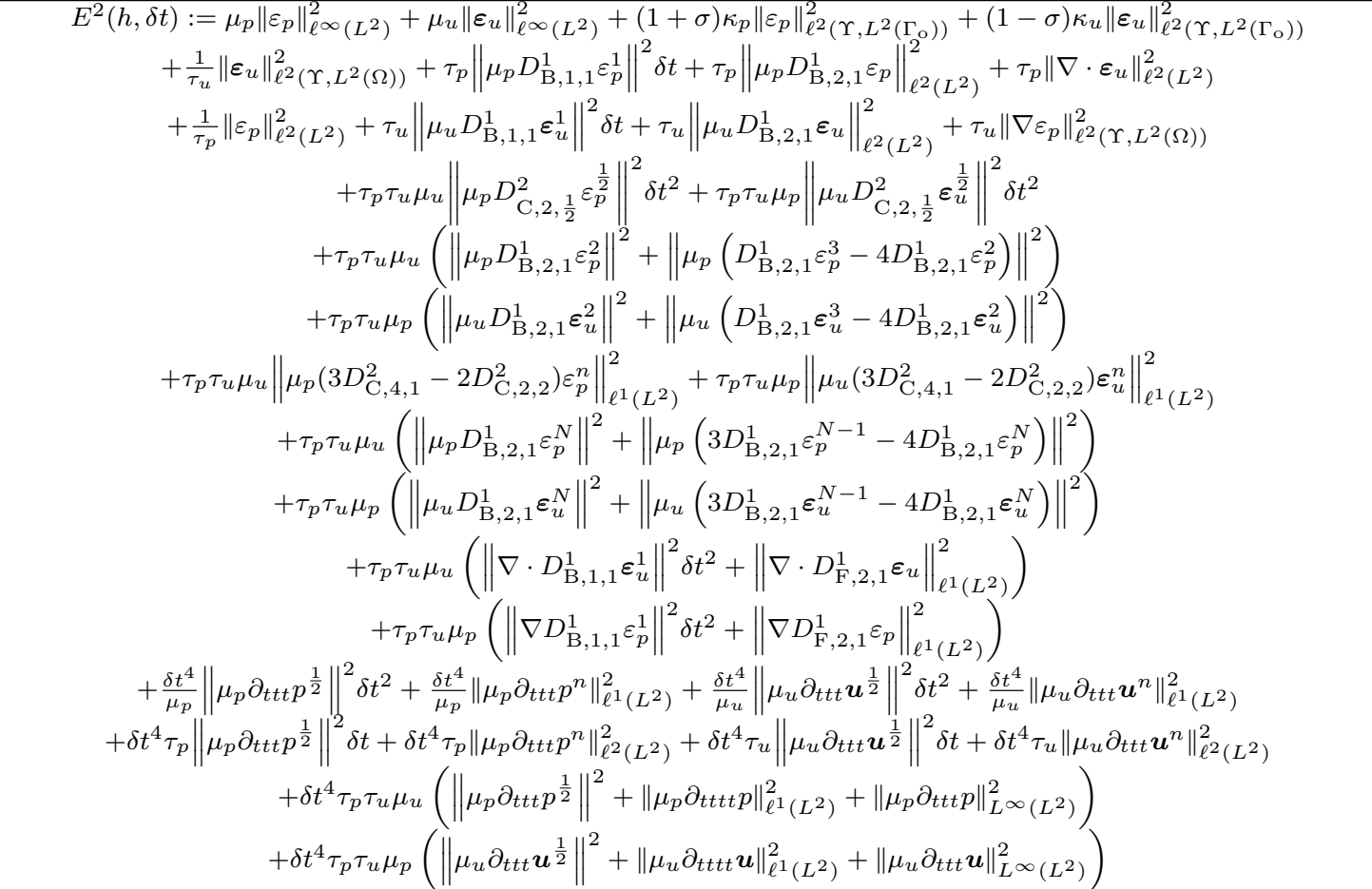 \\
\hline
\end{tabular}

$$
+\tau_{p} \tau_{u} \mu_{u} \sum_{n=1}^{N} \delta t\left(\nabla \cdot \boldsymbol{v}^{n}, \nabla \cdot\left(D_{\mathrm{B}, 1,1}^{1} \boldsymbol{v}^{n}\right)\right)+\tau_{p} \tau_{u} \mu_{p} \sum_{n=1}^{N} \delta t\left(\nabla q^{n}, \nabla\left(D_{\mathrm{B}, 1,1}^{1} q^{n}\right)\right) \gtrsim\left\|V^{N}\right\| \|_{0}^{2} .
$$


Table 5: Error functions II: OSS formulation

\begin{tabular}{|c|c|}
\hline Method & $E(h, \delta t)$ \\
\hline $\mathrm{BE}$ & $\begin{array}{c}E^{2}(h, \delta t):=\mu_{p}\left\|\varepsilon_{p}\right\|_{\ell^{\infty}\left(L^{2}\right)}^{2}+\frac{1}{\tau_{u}}\left\|\varepsilon_{u}\right\|_{\ell^{2}\left(\Upsilon, L^{2}(\Omega)\right)}^{2}+\tau_{p}\left\|\mu_{p} D_{\mathrm{B}, 1,1}^{1} \varepsilon_{p}\right\|_{\ell^{2}\left(L^{2}\right)}^{2}+\tau_{p}\left\|\nabla \cdot \varepsilon_{u}\right\|_{\ell^{2}\left(L^{2}\right)}^{2} \\
+\mu_{u}\left\|\varepsilon_{u}\right\|_{\ell^{\infty}\left(L^{2}\right)}^{2}+\frac{1}{\tau_{p}}\left\|\varepsilon_{p}\right\|_{\ell^{2}\left(L^{2}\right)}^{2}+\tau_{u}\left\|\mu_{u} D_{\mathrm{B}, 1,1}^{1} \varepsilon_{u}\right\|_{\ell^{2}\left(L^{2}\right)}^{2}+\tau_{u}\left\|\nabla \varepsilon_{p}\right\|_{\ell^{2}\left(L^{2}\right)}^{2} \\
+(1+\sigma) \kappa_{p}\left\|\varepsilon_{p}\right\|_{\ell^{2}\left(\Upsilon, L^{2}\left(\Gamma_{\mathrm{o}}\right)\right)}^{2}+(1-\sigma) \kappa_{u}\left\|\varepsilon_{u}\right\|_{\ell^{2}\left(\Upsilon, L^{2}\left(\Gamma_{\mathrm{o}}\right)\right)}^{2} \\
+\frac{\delta t^{2}}{\mu_{p}}\left\|\mu_{p} \partial_{t t} p\right\|_{\ell^{1}\left(L^{2}\right)}^{2}+\frac{\delta t^{2}}{\mu_{u}}\left\|\mu_{u} \partial_{t t} \boldsymbol{u}\right\|_{\ell^{1}\left(L^{2}\right)}^{2}+\delta t^{2} \tau_{p}\left\|\mu_{p} \partial_{t t}\right\|_{\ell^{2}\left(L^{2}\right)}^{2}+\delta t^{2} \tau_{u}\left\|\mu_{u} \partial_{t t} \boldsymbol{u}\right\|_{\ell^{2}\left(L^{2}\right)}^{2}\end{array}$ \\
\hline $\mathrm{CN}$ & $\begin{array}{c}E^{2}(h, \delta t):=\mu_{p}\left\|\varepsilon_{p}\right\|_{\ell^{\infty}\left(L^{2}\right)}^{2}+\mu_{u}\left\|\varepsilon_{u}\right\|_{\ell^{\infty}\left(L^{2}\right)}^{2}+(1+\sigma) \kappa_{p}\left\|\varepsilon_{p}\right\|_{\ell^{2}\left(\Upsilon, L^{2}\left(\Gamma_{\mathrm{o}}\right)\right)}^{2}+(1-\sigma) \kappa_{u}\left\|\varepsilon_{u}\right\|_{\ell^{2}\left(\Upsilon, L^{2}\left(\Gamma_{\mathrm{o}}\right)\right)}^{2} \\
+\frac{1}{\tau_{u}}\left\|\varepsilon_{u}\right\|_{\ell^{2}\left(L^{2}\right)}^{2}+\tau_{p}\left\|\mu_{p} D_{\mathrm{B}, 1,1}^{1} \varepsilon_{p}\right\|_{\ell^{2}\left(L^{2}\right)}^{2}+\tau_{p}\left\|\nabla \cdot \varepsilon_{u}\right\|_{\ell^{2}\left(L^{2}\right)}^{2} \\
\quad+\frac{1}{\tau_{p}}\left\|\varepsilon_{p}\right\|_{\ell^{2}\left(L^{2}\right)}^{2}+\tau_{u}\left\|\mu_{u} D_{\mathrm{B}, 1,1}^{1} \varepsilon_{u}\right\|_{\ell^{2}\left(L^{2}\right)}^{2}+\tau_{u}\left\|\nabla \varepsilon_{p}\right\|_{\ell^{2}\left(L^{2}\right)}^{2} \\
+\frac{\delta t^{4}}{\mu_{p}}\left\|\mu_{p} \partial_{t t t} p\right\|_{\ell^{1}\left(L^{2}\right)}^{2}+\delta t^{4} \tau_{p}\left\|\mu_{p} \partial_{t t t} p\right\|_{\ell^{2}\left(L^{2}\right)}^{2}+\frac{\delta t^{4}}{\mu_{u}}\left\|\mu_{u} \partial_{t t t} \boldsymbol{u}\right\|_{\ell^{1}\left(L^{2}\right)}^{2}+\delta t^{4} \tau_{u}\left\|\mu_{u} \partial_{t t t} \boldsymbol{u}\right\|_{\ell^{2}\left(L^{2}\right)}^{2}\end{array}$ \\
\hline BDF2 & $\begin{aligned} E^{2}(h, \delta t):= & \left.\mu_{p}\left\|\varepsilon_{p}\right\|_{\ell^{\infty}\left(L^{2}\right)}^{2}+\mu_{u}\left\|\varepsilon_{u}\right\|_{\ell^{\infty}\left(L^{2}\right)}^{2}+\frac{1}{\tau_{u}}\left\|\varepsilon_{u}^{\frac{1}{2}}\right\|^{2} \delta t+\frac{1}{\tau_{p}}\left\|\varepsilon_{p}^{\frac{1}{2}}\right\|^{2} \delta t+\frac{1}{\tau_{u}}\left\|\varepsilon_{u}\right\|_{\ell^{2}\left(L^{2}\right)}^{2}+\frac{1}{\tau_{p}}\left\|\varepsilon_{p}\right\|_{\ell^{2}\left(L^{2}\right)}^{2}\right) \\
& +(1+\sigma) \kappa_{p}\left\|\varepsilon_{p}\right\|_{\ell^{2}\left(\Upsilon, L^{2}\left(\Gamma_{\mathrm{o}}\right)\right)}^{2}+(1-\sigma) \kappa_{u}\left\|\varepsilon_{u}\right\|_{\ell^{2}\left(\Upsilon, L^{2}\left(\Gamma_{\mathrm{o}}\right)\right)}^{2} \\
& +\tau_{p}\left\|\mu_{p} D_{\mathrm{B}, 1,1}^{1} \varepsilon_{p}^{1}\right\|^{2} \delta t+\tau_{p}\left\|\nabla \cdot \varepsilon_{u}^{\frac{1}{2}}\right\|^{2} \delta t+\tau_{p}\left\|\mu_{p} D_{\mathrm{B}, 2,1}^{1} \varepsilon_{p}^{n}\right\|_{\ell^{2}\left(L^{2}\right)}^{2}+\tau_{p}\left\|\nabla \cdot \varepsilon_{u}\right\|_{\ell^{2}\left(L^{2}\right)}^{2} \\
& +\tau_{u}\left\|\mu_{u} D_{\mathrm{B}, 1,1}^{1} \varepsilon_{u}^{1}\right\|^{2} \delta t+\tau_{u}\left\|\nabla \varepsilon_{p}^{\frac{1}{2}}\right\|^{2} \delta t+\tau_{u}\left\|\mu_{u} D_{\mathrm{B}, 2,1}^{1} \varepsilon_{u}^{n}\right\|_{\ell^{2}\left(L^{2}\right)}^{2}+\tau_{u}\left\|\nabla \varepsilon_{p}\right\|_{\ell^{2}\left(L^{2}\right)}^{2} \\
+ & \frac{\delta t^{4}}{\mu_{p}}\left\|\mu_{p} \partial_{t t t} p^{\frac{1}{2}}\right\|^{2} \delta t^{2}+\frac{\delta t^{4}}{\mu_{p}}\left\|\mu_{p} \partial_{t t t} p^{n}\right\|_{\ell^{1}\left(L^{2}\right)}^{2}+\frac{\delta t^{4}}{\mu_{u}}\left\|\mu_{p} \partial_{t t t} \boldsymbol{u}^{\frac{1}{2}}\right\|^{2} \delta t^{2}+\frac{\delta t^{4}}{\mu_{u}}\left\|\mu_{u} \partial_{t t t} \boldsymbol{u}^{n}\right\|_{\ell^{1}\left(L^{2}\right)}^{2} \\
+ & \delta t^{4}\left(\tau_{p}\left\|\mu_{p} \partial_{t t t} p^{\frac{1}{2}}\right\|^{2} \delta t+\tau_{p}\left\|\mu_{p} \partial_{t t t} p^{n}\right\|_{\ell^{2}\left(L^{2}\right)}^{2}+\tau_{u}\left\|\mu_{u} \partial_{t t t} \boldsymbol{u}^{\frac{1}{2}}\right\|^{2} \delta t+\tau_{u}\left\|\mu_{u} \partial_{t t t} \boldsymbol{u}^{n}\right\|_{\ell^{2}\left(L^{2}\right)}^{2}\right)\end{aligned}$ \\
\hline
\end{tabular}

The 5th term is bounded using the divergence theorem, splitting the boundary integral in its parts $\Gamma_{p} \Gamma_{u}$ $\Gamma_{\mathrm{o}}$, using the boundary conditions (4) and the definition of $\|V\|_{B}^{2}$ in $(28)$ as

$$
\sum_{n=1}^{N} \delta t\left[\left(\nabla \cdot \boldsymbol{v}^{n}, q^{n}\right)+\left(\nabla q^{n}, \boldsymbol{v}^{n}\right)\right] \geq\|V\|_{B}^{2} .
$$

The 6 th and 7 th terms are already what we need. The 8 th and 9 th terms are greater than zero by boundary conditions. Combining (35)-(37) the proof is completed.

\subsubsection{Proof of stability 31}

Recalling the $\Lambda$-coercivity result we can write

$$
\left\|U_{h}\right\|^{2} \lesssim \mathcal{B}_{h}\left(U_{h}, \Lambda\left(U_{h}\right)\right)=\mathcal{L}_{h}\left(\Lambda\left(U_{h}\right),\right.
$$

and using the definition of $\mathcal{L}_{h}$ we can write

$$
\begin{aligned}
\left\|U_{h}\right\|^{2} \lesssim & \sum_{n=1}^{N} \delta t\left(f_{p}{ }^{n}, p_{h}^{n}+\tau_{p}\left(\mu_{p} D_{\mathrm{B}, 1,1}^{1} p_{h}^{n}+\nabla \cdot \boldsymbol{u}_{h}^{n}\right)\right)+\sum_{n=1}^{N} \delta t\left(f_{p}{ }^{n}, \tau_{p} \nabla \cdot \tau_{u} \mu_{u} D_{\mathrm{B}, 1,1}^{1} \boldsymbol{u}_{h}^{n}\right) \\
& +\sum_{n=1}^{N} \delta t\left(\boldsymbol{f}_{u}{ }^{n}, \boldsymbol{u}_{h}^{n}+\tau_{u}\left(\mu_{u} D_{\mathrm{B}, 1,1}^{1} \boldsymbol{u}_{h}^{n}+\nabla p_{h}^{n}\right)\right)+\sum_{n=1}^{N} \delta t\left(\boldsymbol{f}_{u}{ }^{n}, \tau_{u} \nabla \tau_{p} \mu_{p} D_{\mathrm{B}, 1,1}^{1} p_{h}^{n}\right) .
\end{aligned}
$$

The 1st and 3rd terms can be bounded as

$$
\sum_{n=1}^{N} \delta t\left(f_{p}^{n}, p_{h}^{n}+\tau_{p}\left(\mu_{p} D_{\mathrm{B}, 1,1}^{1} p_{h}^{n}+\nabla \cdot \boldsymbol{u}_{h}^{n}\right)\right)
$$




$$
+\sum_{n=1}^{N} \delta t\left(\boldsymbol{f}_{u}{ }^{n}, \boldsymbol{u}_{h}^{n}+\tau_{u}\left(\mu_{u} D_{\mathrm{B}, 1,1}^{1} \boldsymbol{u}_{h}^{n}+\nabla p_{h}^{n}\right)\right) \lesssim \alpha_{1}\|F\|_{F}^{2}+\frac{1}{\alpha_{1}}\left\|U_{h}\right\|^{2},
$$

where here and in what follows $\alpha_{i}>0$ are reals appearing from Young's inequality. The 2nd and 4 th terms are bounded in a similar manner and we just show how we bound the 2nd term:

$$
\begin{aligned}
\sum_{n=1}^{N} \delta t & \left(f_{p}{ }^{n}, \tau_{p} \nabla \cdot \tau_{u} \mu_{u} D_{\mathrm{B}, 1,1}^{1} \boldsymbol{u}_{h}^{n}\right) \\
= & -\sum_{n=1}^{N} \delta t\left(D_{\mathrm{B}, 1,1}^{1} f_{p}{ }^{n}, \tau_{p} \tau_{u} \mu_{u} \nabla \cdot \boldsymbol{u}_{h}^{n-1}\right)+\left(f_{p}{ }^{N}, \tau_{p} \tau_{u} \mu_{u} \nabla \cdot \boldsymbol{u}_{h}^{N}\right) \\
\lesssim & \alpha_{2} \tau_{p} \tau_{u} \mu_{u}\left\|D_{\mathrm{B}, 1,1}^{1} f_{p}\right\|_{\ell^{1}\left(\Upsilon, L^{2}(\Omega)\right)}^{2}+\alpha_{3} \tau_{p} \tau_{u} \mu_{u}\left\|f_{p}\right\|_{\ell^{\infty}\left(\Upsilon, L^{2}(\Omega)\right)}^{2} \\
& +\frac{1}{\alpha_{2}} \tau_{p} \tau_{u} \mu_{u}\left\|\nabla \cdot \boldsymbol{u}_{h}\right\|_{\ell^{\infty}\left(\Upsilon, L^{2}(\Omega)\right)}^{2}+\frac{1}{\alpha_{3}} \tau_{p} \tau_{u} \mu_{u}\left\|\nabla \cdot \boldsymbol{u}_{h}\right\|_{\ell^{\infty}\left(\Upsilon, L^{2}(\Omega)\right)}^{2} .
\end{aligned}
$$

Combining (38)-(41) and taking $\alpha_{i}$ large enough the proof is complete.

\subsubsection{Proof of convergence 32}

Combining $\Lambda$-coercivity with $V=e$ and (34) we can arrive to

$$
\|e\|^{2} \lesssim \mathcal{B}_{h}(e, \Lambda(e))=\mathcal{B}_{h}(\varepsilon, \Lambda(e))+\mathcal{C}(U, \Lambda(e)) .
$$

The aim is to bound $e$ in terms of $\varepsilon$ and $U$. We first analyze the term containing $\mathcal{B}_{h}$ and then the term associated to the consistency error (33):

$$
\begin{aligned}
\mathcal{B}_{h}(\varepsilon, \Lambda(e))= & \sum_{n=1}^{N} \delta t\left(\mu_{p} D_{\mathrm{B}, 1,1}^{1} \varepsilon_{p}^{n}+\nabla \cdot \varepsilon_{u}^{n}, e_{p}^{n}+\tau_{p}\left(\mu_{p} D_{\mathrm{B}, 1,1}^{1} e_{p}^{n}+\nabla \cdot \boldsymbol{e}_{u}^{n}\right)+\tau_{p} \tau_{u} \mu_{u} \nabla \cdot D_{\mathrm{B}, 1,1}^{1} \boldsymbol{e}_{u}^{n}\right) \\
& +\sum_{n=1}^{N} \delta t\left(\mu_{u} D_{\mathrm{B}, 1,1}^{1} \varepsilon_{u}^{n}+\nabla \varepsilon_{p}^{n}, \boldsymbol{e}_{u}^{n}+\tau_{u}\left(\mu_{u} D_{\mathrm{B}, 1,1}^{1} \boldsymbol{e}_{u}^{n}+\nabla e_{p}^{n}\right),+\tau_{p} \tau_{u} \mu_{p} \nabla D_{\mathrm{B}, 1,1}^{1} e_{p}^{n}\right) \\
\leq & \sum_{n=1}^{N} \delta t\left[\frac{\alpha_{1}}{2 \tau_{p}}\left\|\varepsilon_{p}^{n}\right\|^{2}+\frac{\tau_{p}}{2 \alpha_{1}}\left\|\mu_{p} D_{\mathrm{B}, 1,1}^{1} e_{p}^{n}+\nabla \cdot \boldsymbol{e}_{u}^{n}\right\|^{2}\right] \\
& +\sum_{n=1}^{N} \delta t\left[\frac{\alpha_{2}}{2 \tau_{u}}\left\|\varepsilon_{u}^{n}\right\|^{2}+\frac{\tau_{u}}{2 \alpha_{2}}\left\|\mu_{u} D_{\mathrm{B}, 1,1}^{1} e_{u}^{n}+\nabla e_{p}^{n}\right\|^{2}\right] \\
& +\sum_{n=1}^{N} \delta t \tau_{p}\left[\frac{\alpha_{3}}{2}\left\|\mu_{p} D_{\mathrm{B}, 1,1}^{1} \varepsilon_{p}^{n}+\nabla \cdot \varepsilon_{u}^{n}\right\|^{2}+\frac{1}{2 \alpha_{3}}\left\|\mu_{p} D_{\mathrm{B}, 1,1}^{1} e_{p}^{n}+\nabla \cdot \boldsymbol{e}_{u}^{n}\right\|^{2}\right] \\
& +\sum_{n=1}^{N} \delta t \tau_{u}\left[\frac{\alpha_{4}}{2}\left\|\mu_{u} D_{\mathrm{B}, 1,1}^{1} \varepsilon_{u}^{n}+\nabla \varepsilon_{p}^{n}\right\|^{2}+\frac{1}{2 \alpha_{4}}\left\|\mu_{u} D_{\mathrm{B}, 1,1}^{1} \boldsymbol{e}_{u}^{n}+\nabla e_{p}^{n}\right\|^{2}\right] \\
& +\sum_{n=1}^{N} \delta t\left(\mu_{p} D_{\mathrm{B}, 1,1}^{1} \varepsilon_{p}^{n}+\nabla \cdot \varepsilon_{u}^{n}, \tau_{p} \tau_{u} \mu_{u} \nabla \cdot D_{\mathrm{B}, 1,1}^{1} e_{u}^{n}\right) \\
& +\sum_{n=1}^{N} \delta t\left(\mu_{u} D_{\mathrm{B}, 1,1}^{1} \varepsilon_{u}^{n}+\nabla \varepsilon_{p}^{n+1}, \tau_{p} \tau_{u} \mu_{p} \nabla D_{\mathrm{B}, 1,1}^{1} e_{p}^{n}\right) .
\end{aligned}
$$

The only terms missing to bound are the last two ones. Both are bound similarly and we will only show how the first one is bounded. The first part is bounded as

$$
\sum_{n=1}^{N} \delta t\left(\mu_{p} D_{\mathrm{B}, 1,1}^{1} \varepsilon_{p}^{n}, \tau_{p} \tau_{u} \mu_{u} \nabla \cdot D_{\mathrm{B}, 1,1}^{1} e_{u}^{n}\right)
$$




$$
\begin{aligned}
& =\tau_{p} \tau_{u} \mu_{u}\left[\left(\mu_{p} D_{\mathrm{B}, 1,1}^{1} \varepsilon_{p}^{N}, \nabla \cdot \boldsymbol{e}_{u}^{N}\right)-\left(\mu_{p} D_{\mathrm{B}, 1,1}^{1} \varepsilon_{p}^{1}, \nabla \cdot \boldsymbol{e}_{u}^{0}\right)\right]-\sum_{n=1}^{N-1} \delta t \tau_{p} \tau_{u} \mu_{u}\left(\mu_{p} D_{\mathrm{C}, 2,1}^{2} \varepsilon_{p}^{n}, \nabla \cdot \boldsymbol{e}_{u}^{n}\right) \\
& \leq \tau_{p} \tau_{u} \mu_{u}\left[\frac{\alpha_{5}}{2}\left\|\mu_{p} D_{\mathrm{B}, 1,1}^{1} \varepsilon_{p}^{N}\right\|^{2}+\frac{1}{2 \alpha_{5}}\left\|\nabla \cdot \boldsymbol{e}_{u}^{N}\right\|^{2}\right]+\tau_{p} \tau_{u} \mu_{u}\left[\frac{\alpha_{6}}{2}\left\|\mu_{p} D_{\mathrm{C}, 2,1}^{2} \varepsilon_{p}\right\|_{\ell^{1}\left(L^{2}\right)}^{2}+\frac{1}{2 \alpha_{6}}\left\|\nabla \cdot \boldsymbol{e}_{u}\right\|_{\ell^{\infty}\left(L^{2}\right)}^{2}\right],
\end{aligned}
$$

and the second part is bounded as

$$
\begin{aligned}
& \sum_{n=1}^{N} \delta t\left(\nabla \cdot \varepsilon_{u}^{n}, \tau_{p} \tau_{u} \mu_{u} \nabla \cdot D_{\mathrm{B}, 1,1}^{1} \boldsymbol{e}_{u}^{n}\right) \\
& \quad=\tau_{p} \tau_{u} \mu_{u}\left[\left(\nabla \cdot \varepsilon_{u}^{N}, \nabla \cdot \boldsymbol{e}_{u}^{N}\right)-\left(\nabla \cdot \varepsilon_{u}^{0}, \nabla \cdot \boldsymbol{e}_{u}^{0}\right)\right]-\sum_{n=1}^{N} \delta t\left(\nabla \cdot D_{\mathrm{B}, 1,1}^{1} \varepsilon_{u}^{n}, \tau_{p} \tau_{u} \mu_{u} \nabla \cdot \boldsymbol{e}_{u}^{n}\right) \\
& \quad \leq \tau_{p} \tau_{u} \mu_{u}\left[\frac{\alpha_{7}}{2}\left\|\nabla \cdot \varepsilon_{u}^{N}\right\|^{2}+\frac{1}{2 \alpha_{7}}\left\|\nabla \cdot \boldsymbol{e}_{u}^{N}\right\|^{2}+\frac{\alpha_{8}}{2}\left\|\nabla \cdot D_{\mathrm{B}, 1,1}^{1} \varepsilon_{u}\right\|_{\ell^{1}\left(L^{2}\right)}^{2}+\frac{1}{2 \alpha_{8}}\left\|\nabla \cdot \boldsymbol{e}_{u}\right\|_{\ell^{\infty}\left(L^{2}\right)}^{2}\right] .
\end{aligned}
$$

This completes the bounding of $\mathcal{B}_{h}$. Now let us bound the consistency error (33):

$$
\begin{aligned}
\mathcal{C}(U, \Lambda(e))=\mathcal{L}_{h}(\Lambda(e))-\mathcal{B}_{h}(U, \Lambda(e)) & \\
= & -\sum_{n=1}^{N} \delta t\left(\mu_{p} D_{\mathrm{B}, 1,1}^{1} p^{n}+\nabla \cdot \boldsymbol{u}^{n}, e_{p}^{n}+\tau_{p}\left(\mu_{p} D_{\mathrm{B}, 1,1}^{1} e_{p}^{n}+\nabla \cdot \boldsymbol{e}_{u}^{n}\right)+\tau_{p} \tau_{u} \mu_{u} \nabla \cdot D_{\mathrm{B}, 1,1}^{1} \boldsymbol{e}_{u}^{n}\right) \\
& -\sum_{n=1}^{N} \delta t\left(\mu_{u} D_{\mathrm{B}, 1,1}^{1} \boldsymbol{u}^{n}+\nabla p^{n}, \boldsymbol{e}_{u}^{n}+\tau_{u}\left(\mu_{u} D_{\mathrm{B}, 1,1}^{1} \boldsymbol{e}_{u}^{n}+\nabla e_{p}^{n}\right)+\tau_{p} \tau_{u} \mu_{p} \nabla D_{\mathrm{B}, 1,1}^{1} e_{p}^{n}\right) .
\end{aligned}
$$

Both terms are bounded similarly and we only show how it is bounded the first one. Let us start with its first part

$$
\begin{aligned}
-\sum_{n=1}^{N} \delta t\left(\mu_{p} D_{\mathrm{B}, 1,1}^{1} p^{n}+\nabla \cdot \boldsymbol{u}^{n}, e_{p}^{n}\right) & \leq \frac{\alpha_{9}}{2} \frac{\delta t^{2}}{\mu_{p}}\left[\sum_{n=1}^{N}\left\|\mu_{p} D_{\mathrm{B}, 1,1}^{1} p^{n}+\nabla \cdot \boldsymbol{u}^{n}\right\|\right]^{2}+\frac{1}{2 \alpha_{9}} \mu_{p}\left\|e_{p}\right\|_{\ell^{\infty}\left(L^{2}\right)}^{2} \\
& \leq \frac{\alpha_{9}}{2} \frac{\delta t^{2}}{\mu_{p}}\left[\sum_{n=1}^{N}\left\|\mu_{p} D_{\mathrm{B}, 1,1}^{1} p^{n}-\mu_{p} \partial_{t} p^{n}\right\|\right]^{2}+\frac{1}{2 \alpha_{9}} \mu_{p}\left\|e_{p}\right\|_{\ell^{\infty}\left(L^{2}\right)}^{2} \\
& \leq \frac{\alpha_{9}}{2} \frac{\delta t^{2}}{\mu_{p}}\left\|\mu_{p} \partial_{t t} p^{n}\right\|_{\ell^{1}\left(L^{2}\right)}^{2}+\frac{1}{2 \alpha_{9}} \mu_{p}\left\|e_{p}\right\|_{\ell \infty\left(L^{2}\right)}^{2} .
\end{aligned}
$$

The second part can be bounded as

$$
\begin{aligned}
-\sum_{n=1}^{N} & \delta t \\
\quad \leq & \frac{\alpha_{10}}{2} \sum_{n=1}^{N} \delta t \tau_{p}\left\|\mu_{p} D_{\mathrm{B}, 1,1}^{1} p^{n}-\nabla \cdot \mu_{p} \partial_{t} p^{n}\right\|^{2}+\frac{1}{2 \alpha_{10}} \sum_{n=1}^{N} \delta t \tau_{p}\left\|\mu_{p} D_{\mathrm{B}, 1,1}^{1} e_{p}^{n}+\nabla \cdot \boldsymbol{e}_{u}^{n}\right\|^{2} \\
\quad & \leq \frac{\alpha_{10}}{2} \delta t^{2} \tau_{p}\left\|\mu_{p} \partial_{t t} p^{n}\right\|_{\ell^{2}\left(\Upsilon, L^{2}(\Omega)\right)}^{2}+\frac{1}{2 \alpha_{10}} \tau_{p}\left\|\mu_{p} D_{\mathrm{B}, 1,1}^{1} e_{p}^{n}+\nabla \cdot \boldsymbol{e}_{u}^{n}\right\|_{\ell^{2}\left(\Upsilon, L^{2}(\Omega)\right)}^{2} .
\end{aligned}
$$

The third part can be bounded as

$$
-\sum_{n=1}^{N} \delta t\left(\mu_{p} D_{\mathrm{B}, 1,1}^{1} p^{n}+\nabla \cdot \boldsymbol{u}^{n}, \tau_{p} \tau_{u} \mu_{u} \nabla \cdot D_{\mathrm{B}, 1,1}^{1} \boldsymbol{e}_{u}^{n}\right)
$$




$$
\begin{aligned}
= & \tau_{p} \tau_{u} \mu_{u}\left[\left(\mu_{p} D_{\mathrm{B}, 1,1}^{1} p^{N}+\nabla \cdot \boldsymbol{u}^{N}, \nabla \cdot \boldsymbol{e}_{u}^{N}\right)-\left(\mu_{p} D_{\mathrm{B}, 1,1}^{1} p^{1}+\nabla \cdot \boldsymbol{u}^{0}, \nabla \cdot \boldsymbol{e}_{u}^{0}\right)\right] \\
& -\tau_{p} \tau_{u} \mu_{u}\left[\sum_{n=1}^{N-1} \delta t\left(\mu_{p} D_{\mathrm{C}, 2,1}^{2} p^{n}+\nabla \cdot D_{\mathrm{B}, 1,1}^{1} \boldsymbol{u}^{n}, \nabla \cdot \boldsymbol{e}_{u}^{n}\right)+\delta t\left(\nabla \cdot D_{\mathrm{B}, 1,1}^{1} \boldsymbol{u}^{1}, \nabla \cdot \boldsymbol{e}_{u}^{0}\right)\right] \\
\leq & \tau_{p} \tau_{u} \mu_{u}\left[\frac{\alpha_{11}}{2}\left\|\mu_{p} D_{\mathrm{B}, 1,1}^{1} p^{N}+\nabla \cdot \boldsymbol{u}^{N}\right\|^{2}+\frac{1}{2 \alpha_{11}}\left\|\nabla \cdot \boldsymbol{e}_{u}^{N}\right\|^{2}\right] \\
& +\tau_{p} \tau_{u} \mu_{u} \frac{\alpha_{12}}{2}\left[\sum_{n=1}^{N-1} \delta t\left\|\mu_{p} D_{\mathrm{C}, 2,1}^{2} p^{n}+\nabla \cdot D_{\mathrm{B}, 1,1}^{1} \boldsymbol{u}^{n}\right\|\right]^{2}+\tau_{p} \tau_{u} \mu_{u} \frac{1}{2 \alpha_{12}}\left\|\nabla \cdot \boldsymbol{e}_{u}\right\|^{2} \\
\leq & \tau_{p} \tau_{u} \mu_{u}\left[\frac{\alpha_{11}}{2} \delta t^{2}\left\|\mu_{p} \partial_{t t} p^{N}\right\|^{2}+\frac{1}{2 \alpha_{11}}\left\|\nabla \cdot \boldsymbol{e}_{u}^{N}\right\|^{2}\right] \\
& +\tau_{p} \tau_{u} \mu_{u}\left[\frac{\alpha_{12}}{2} \delta t^{2}\left\|\mu_{p} \partial_{t t t} p\right\|_{\ell^{1}\left(\Upsilon, L^{2}(\Omega)\right)}^{2}+\frac{1}{2 \alpha_{12}}\left\|\nabla \cdot \boldsymbol{e}_{u}\right\|_{\ell^{\infty}\left(\Upsilon, L^{2}(\Omega)\right)}^{2}\right] .
\end{aligned}
$$

Combining all bounds the proof is complete.

\subsection{Accuracy of the fully discrete methods}

Let $k$ be the order of $p$-interpolation and $l$ the order of $\boldsymbol{u}$-interpolation. Analyzing the a priori error estimates $(32)$ for the fully discrete methods with the error functions given in Tables 4 and 5 and assuming regular enough solutions, we can summarize the convergence rates as shown in Tables 6 8 . We stress the fact that the convergence rates do depend on the choice of the stabilization parameters, and different convergence orders are obtained for the three discrete variational formulations above. Let us note that the time discretization schemes do not spoil the spatial convergence rates obtained in [10, 11] for the timecontinuous case. Let us mention that the negative powers of $h$ appear due to some terms in the error function having $\tau_{p}$ or $\tau_{u}$ dividing (see See Tables 4 and 5 . Obviously, an appropriate $(\delta t, h)$ relationship should be chosen to have convergence.

Table 6: Convergence rates for ASGS-BE and OSS-BE according to variational form

\begin{tabular}{|c|c|c|c|}
\hline Variational Form & I & II & III \\
\hline$\left\|\xi_{p}^{n}\right\|_{\ell \infty\left(L^{2}\right)},\left\|\boldsymbol{\xi}_{u}^{n}\right\|_{\ell \infty}\left(L^{2}\right)$ & $h^{k+\frac{1}{2}}+h^{l+\frac{1}{2}}+\delta t+h^{\frac{1}{2}} \delta t$ & $h^{k+\frac{1}{2}}+h^{l}+\delta t$ & $h^{k}+h^{l+\frac{1}{2}}+\delta t$ \\
\hline$\left\|\mu_{u} D_{\mathrm{B}, 1,1}^{1} \boldsymbol{\xi}_{u}^{n}+\nabla \xi_{p}^{n}\right\|_{\ell^{2}\left(L^{2}\right)}$ & $h^{k}+h^{l}+h^{-\frac{1}{2}} \delta t+\delta t$ & $h^{k-\frac{1}{2}}+h^{l-1}+h^{-1} \delta t$ & $h^{k}+h^{l+\frac{1}{2}}+\delta t$ \\
\hline$\left\|\mu_{p} D_{\mathrm{B}, 1,1}^{1} \xi_{p}^{n}+\nabla \cdot \boldsymbol{\xi}_{u}^{n}\right\|_{\ell^{2}\left(L^{2}\right)}$ & $h^{k}+h^{l}+h^{-\frac{1}{2}} \delta t+\delta t$ & $h^{k+\frac{1}{2}}+h^{l}+\delta t$ & $h^{k-1}+h^{l-\frac{1}{2}}+h^{-1} \delta t$ \\
\hline Best $k, l, h-\delta t$ & $k=l, \delta t \sim h^{k+\frac{1}{2}}$ & $k+\frac{1}{2}=l, \delta t \sim h^{l}$ & $k=l+\frac{1}{2}, \delta t \sim h^{k}$ \\
& & $k=l, \delta t \sim h^{l}$ & $k=l, \delta t \sim h^{k}$ \\
& & $k+1=l, \delta t \sim h^{l-\frac{1}{2}}$ & $k=l+1, \delta t \sim h^{k-\frac{1}{2}}$ \\
\hline
\end{tabular}

\section{Fourier analysis}

We show now the results of a Fourier analysis (or von Neumann analysis) in 1D using linear (P1) elements, which serves to study dispersion, dissipation, and stability of the numerical schemes. The analysis is done in an unbounded domain with no forcing terms, but with non-zero initial conditions. The mesh is taken uniform, of size $h$, and the time step is $\delta t$. We focus on variational form I because it has the best convergence properties, as shown in the previous analysis. 
Table 7: Convergence rates for ASGS-CN and OSS-CN according to variational form

\begin{tabular}{|c|c|c|c|}
\hline Variational Form & I & II & III \\
\hline$\left\|\xi_{p}^{n}\right\|_{\ell^{\infty}\left(L^{2}\right)},\left\|\boldsymbol{\xi}_{u}^{n}\right\|_{\ell \infty}\left(L^{2}\right)$ & $h^{k+\frac{1}{2}}+h^{l+\frac{1}{2}}+\delta t^{2}+h^{\frac{1}{2}} \delta t^{2}$ & $h^{k+\frac{1}{2}}+h^{l}+\delta t^{2}$ & $h^{k}+h^{l+\frac{1}{2}}+\delta t^{2}$ \\
\hline$\left\|\mu_{u} D_{\mathrm{B}, 1,1}^{1} \boldsymbol{\xi}_{u}^{n}+\nabla \xi_{p}^{n-\frac{1}{2}}\right\|_{\ell^{2}\left(L^{2}\right)}$ & $h^{k}+h^{l}+h^{-\frac{1}{2}} \delta t^{2}+\delta t^{2}$ & $h^{k-\frac{1}{2}}+h^{l-1}+h^{-1} \delta t^{2}$ & $h^{k}+h^{l+\frac{1}{2}}+\delta t^{2}$ \\
\hline$\left\|\mu_{p} D_{\mathrm{B}, 1,1}^{1} \xi_{p}^{n}+\nabla \cdot \boldsymbol{\xi}_{u}^{n-\frac{1}{2}}\right\|_{\ell^{2}\left(L^{2}\right)}$ & $h^{k}+h^{l}+h^{-\frac{1}{2}} \delta t^{2}+\delta t^{2}$ & $h^{k+\frac{1}{2}}+h^{l}+\delta t^{2}$ & $h^{k-1}+h^{l-\frac{1}{2}}+h^{-1} \delta t^{2}$ \\
\hline Best $k, l, h-\delta t$ & $k=l, \delta t^{2} \sim h^{k+\frac{1}{2}}$ & $k+\frac{1}{2}=l, \delta t^{2} \sim h^{l}$ & $k=l+\frac{1}{2}, \delta t^{2} \sim h^{k}$ \\
& & $k=l, \delta t^{2} \sim h^{l}$ & $k=l, \delta t^{2} \sim h^{k}$ \\
& & $k+1=l, \delta t^{2} \sim h^{l-\frac{1}{2}}$ & $k=l+1, \delta t^{2} \sim h^{k-\frac{1}{2}}$ \\
\hline
\end{tabular}

Table 8: Convergence rates for ASGS-BDF2 and OSS-BDF2 according to variational form

\begin{tabular}{|c|c|c|c|}
\hline Variational Form & I & II & III \\
\hline$\left\|\xi_{p}^{n}\right\|_{\ell^{\infty}\left(L^{2}\right)},\left\|\boldsymbol{\xi}_{u}^{n}\right\|_{\ell^{\infty}\left(L^{2}\right)}$ & $h^{k+\frac{1}{2}}+h^{l+\frac{1}{2}}+\delta t^{2}$ & $h^{k+\frac{1}{2}}+h^{l}+\delta t^{2}$ & $h^{k}+h^{l+\frac{1}{2}}+\delta t^{2}$ \\
\hline$\left\|\mu_{u} D_{\mathrm{B}, 2,1}^{1} \boldsymbol{\xi}_{u}^{n}+\nabla \xi_{p}^{n}\right\|_{\ell^{2}\left(L^{2}\right)}$ & $h^{k}+h^{l}+h^{-\frac{1}{2}} \delta t^{2}$ & $h^{k-\frac{1}{2}}+h^{l-1}+h^{-1} \delta t^{2}$ & $h^{k}+h^{l+\frac{1}{2}}+\delta t^{2}$ \\
\hline$\left\|\mu_{p} D_{\mathrm{B}, 2,1}^{1} \xi_{p}^{n}+\nabla \cdot \boldsymbol{\xi}_{u}^{n}\right\|_{\ell^{2}\left(L^{2}\right)}$ & $h^{k}+h^{l}+h^{-\frac{1}{2}} \delta t^{2}$ & $h^{k+\frac{1}{2}}+h^{l}+\delta t^{2}$ & $h^{k-1}+h^{l-\frac{1}{2}}+h^{-1} \delta t^{2}$ \\
\hline $\operatorname{Best} k, l, h-\delta t$ & $k=l, \delta t^{2} \sim h^{k+\frac{1}{2}}$ & $k+\frac{1}{2}=l, \delta t^{2} \sim h^{l}$ & $k=l+\frac{1}{2}, \delta t^{2} \sim h^{k}$ \\
& & $k=l, \delta t^{2} \sim h^{l}$ & $k=l, \delta t^{2} \sim h^{k}$ \\
& & $k+1=l, \delta t^{2} \sim h^{l-\frac{1}{2}}$ & $k=l+1, \delta t^{2} \sim h^{k-\frac{1}{2}}$ \\
\hline
\end{tabular}

Let us consider a solution of the form $p=C_{p} \mathrm{e}^{\mathrm{i}(k x-\omega t)}$ and $u=C_{p} \mu_{p}^{1 / 2} \mu_{u}^{-1 / 2} \mathrm{e}^{\mathrm{i}(k x-\omega t)}$, where $C_{p}$ is an arbitrary constant such that $[p]=\left[C_{p}\right],[\cdot]$ standing for dimensional group, $\omega$ is the angular frequency (temporal frequency) and $k$ is the (angular) wavenumber (spatial frequency). It can be checked that this plane wave is solution of the wave equation in mixed form. The angular frequency and wavenumber are related through the wave speed as $\omega=k c$.

We denote by $\Re(\cdot)$ and $\Im(\cdot)$ to the real and imaginary parts of a complex number respectively. The stability conditions can be summarized as follows: $\Im(k h) \geq 0$ or $\Im(\omega \delta t) \leq 0$, where $k$ and $\omega$ are the semi-discrete or fully-discrete wavenumber and angular frequency respectively.

For 1D P1 elements of size diam $K=h$ we will need the following element matrices:

$$
\begin{aligned}
& \left.\int_{K} N_{i} N_{j}\right|_{i, j=1,2}=\frac{h}{6}\left(\begin{array}{ll}
2 & 1 \\
1 & 2
\end{array}\right),\left.\quad \int_{K} N_{i} \partial_{x} N_{j}\right|_{i, j=1,2}=\frac{1}{2}\left(\begin{array}{ll}
-1 & 1 \\
-1 & 1
\end{array}\right) \\
& \left.\int_{K} \partial_{x} N_{i} N_{j}\right|_{i, j=1,2}=\frac{1}{2}\left(\begin{array}{cc}
-1 & -1 \\
1 & 1
\end{array}\right),\left.\quad \int_{K} \partial_{x} N_{i} \partial_{x} N_{j}\right|_{i, j=1,2}=\frac{1}{h}\left(\begin{array}{cc}
1 & -1 \\
-1 & 1
\end{array}\right),
\end{aligned}
$$

where $N_{i}$ is the shape function of node $i$ (in element $K$ ). When assembled for just two elements sharing a generic node they give, respectively,

$$
M_{p}=M_{u}=\frac{h}{6}\left(\begin{array}{lll}
2 & 1 & 0 \\
1 & 4 & 1 \\
0 & 2 & 1
\end{array}\right), \quad K_{p}=K_{u}=\frac{1}{2}\left(\begin{array}{ccc}
-1 & 1 & 0 \\
-1 & 0 & 1 \\
0 & -1 & 1
\end{array}\right),
$$



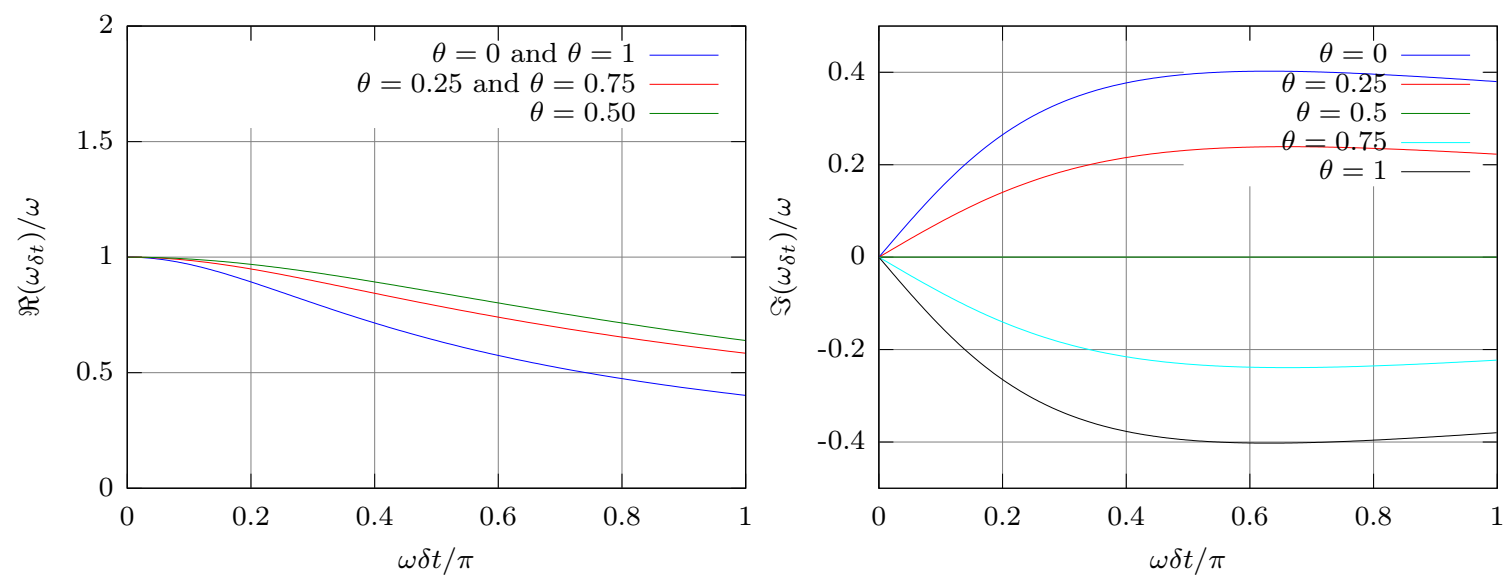

Figure 1: $\theta$-method time semi-discretization
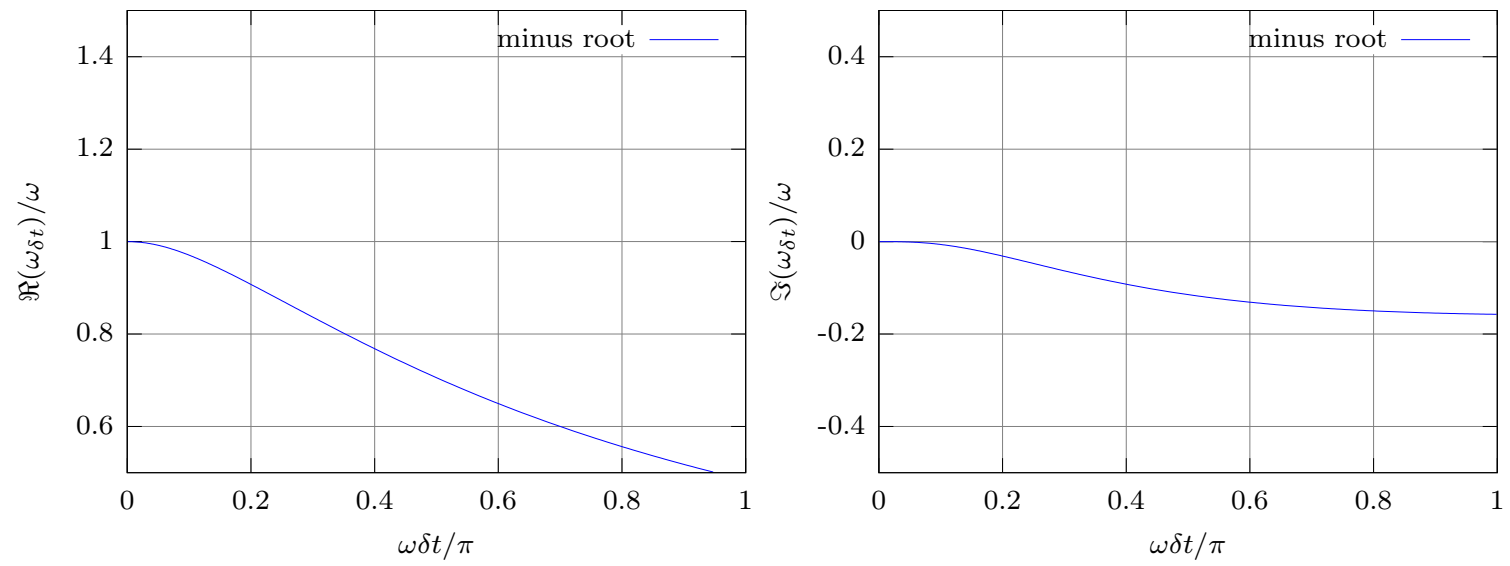

Figure 2: BDF2 time semi-discretization

$$
M_{S, p}=M_{S, u}=\frac{1}{2}\left(\begin{array}{ccc}
-1 & -1 & 0 \\
1 & 0 & -1 \\
0 & 1 & 1
\end{array}\right), \quad K_{S, p}=K_{S, u}=\frac{1}{h}\left(\begin{array}{ccc}
1 & -1 & 0 \\
-1 & 2 & -1 \\
0 & -1 & 1
\end{array}\right) .
$$

Additionally, we define the lumped mass matrices

$$
\widetilde{M}_{p}=\widetilde{M}_{u}=\frac{h}{2}\left(\begin{array}{lll}
1 & 0 & 0 \\
0 & 2 & 0 \\
0 & 0 & 1
\end{array}\right) .
$$

First we will analyze the time semi-discretization, then the space semi-discretization and finally the full discretization. The time discretizations to be considered are the $\theta$ and BDF2 methods, whereas the space discretizations to be analyzed are stabilized FE methods (ASGS and OSS). We will often take $t_{n}=n \delta t$ and $x_{j}=j h$, where $n$ is the time step and $j$ is the mesh point.

\subsection{Time semi-discretization}

Let us start considering the effect of the time discretization only, without discretizing in space. Let us take $\omega_{\delta t}=k_{\delta t} c_{\delta t}$ and $k_{\delta t}=k$, where the subscript $\delta t$ denotes temporal semi-discretization. We take a solution (mode) of the form

$$
\left[P^{n}(x), U^{n}(x)\right]=\phi_{k} \mathrm{e}^{\mathrm{i}\left(k x-\omega_{\delta t} t_{n}\right)}\left[1, \mu_{p}^{1 / 2} \mu_{u}^{-1 / 2}\right],
$$



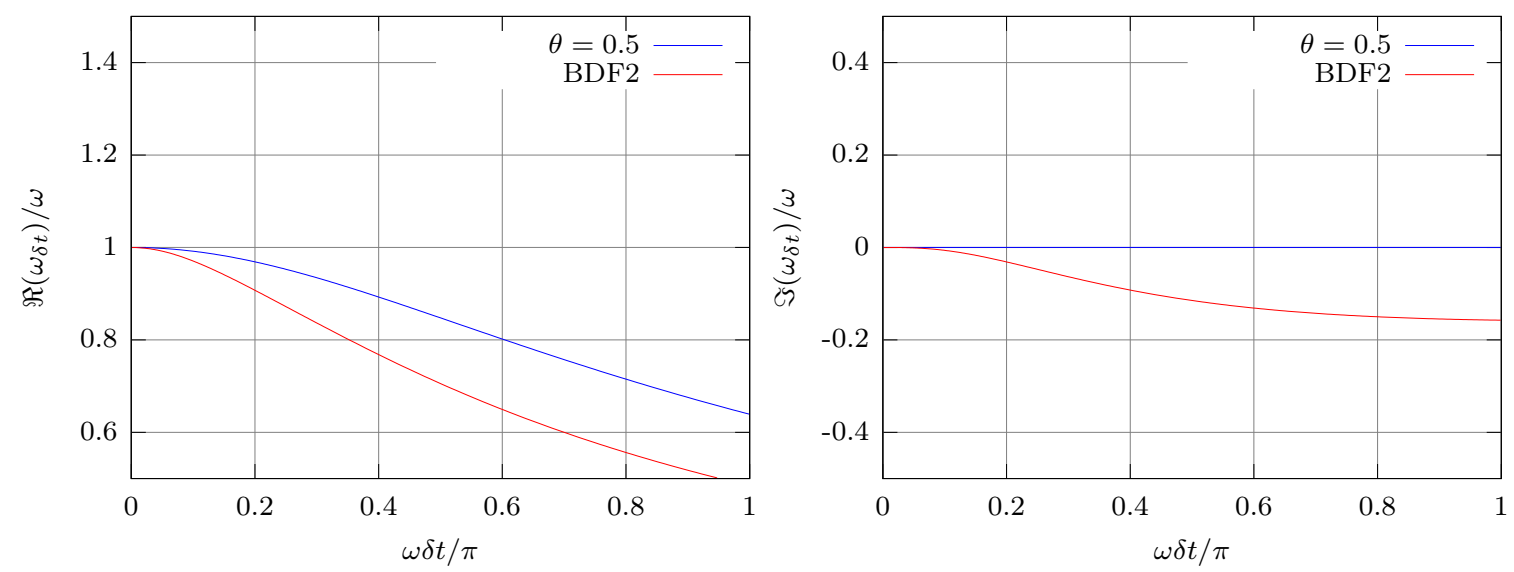

Figure 3: Time semi-discretization comparison

where $\phi_{k}$ is a constant that describes the amplitude at $t_{n}=0$.

The semi-discrete problem using the $\theta$ method is as follows:

$$
\begin{aligned}
& \frac{\mu_{p}}{\delta t}\left(P^{n+1}-P^{n}\right)+\theta \frac{\mathrm{d}}{\mathrm{d} x} U^{n+1}+(1-\theta) \frac{\mathrm{d}}{\mathrm{d} x} U^{n}=0, \\
& \frac{\mu_{u}}{\delta t}\left(U^{n+1}-U^{n}\right)+\theta \frac{\mathrm{d}}{\mathrm{d} x} P^{n+1}+(1-\theta) \frac{\mathrm{d}}{\mathrm{d} x} P^{n}=0,
\end{aligned}
$$

where $0 \leq \theta \leq 1$ is a parameter. The backward Euler method corresponds to $\theta=1$, the forward Euler method to $\theta=0$ and the trapezoidal rule to $\theta=0.5$. Replacing $\left[P^{n}, U^{n}\right]$ from (42) we have:

$$
\begin{aligned}
& \frac{\mu_{p}}{\delta t}\left(\mathrm{e}^{-\mathrm{i} \omega_{\delta t} \delta t}-1\right)+\mu_{p}^{1 / 2} \mu_{u}^{-1 / 2}\left(\mathrm{i} k \theta \mathrm{e}^{-\mathrm{i} \omega_{\delta t} \delta t}+\mathrm{i} k(1-\theta)\right)=0, \\
& \frac{\mu_{u}}{\delta t} \mu_{p}^{1 / 2} \mu_{u}^{-1 / 2}\left(\mathrm{e}^{-\mathrm{i} \omega_{\delta t} \delta t}-1\right)+\mathrm{i} k \theta \mathrm{e}^{-\mathrm{i} \omega_{\delta t} \delta t}+\mathrm{i} k(1-\theta)=0 .
\end{aligned}
$$

Both equations are equivalent, so we just analyze one of them. We have that

$$
\omega_{\delta t} \delta t=\frac{-1}{\mathrm{i}} \log \left(\frac{1-(1-\theta) \mathrm{i} \omega \delta t}{1+\mathrm{i} \omega \delta t \theta}\right)
$$

The numerical angular frequency, $\omega_{\delta t}$, is not always real, it is complex for $\theta \neq 0.5$. It can be shown that the angular frequency error is 2 nd order in $\omega \delta t$ for $\theta=0.5$ and only 1 st order in $\omega \delta t$ for $\theta \neq 0.5$. Fig. 1 shows the angular frequency ratio $\omega_{\delta t} / \omega$ for $0 \leq \omega \delta t / \pi \leq 1$. It can be seen that the $\theta$-method is unconditionally stable for $\theta \geq 0.5$.

On the other hand, the semi-discrete problem using BDF2 is:

$$
\begin{aligned}
& \frac{\mu_{p}}{2 \delta t}\left(3 P^{n+1}-4 P^{n}+P^{n-1}\right)+\frac{\mathrm{d}}{\mathrm{d} x} U^{n+1}=0, \\
& \frac{\mu_{u}}{2 \delta t}\left(3 U^{n+1}-4 U^{n}+U^{n-1}\right)+\frac{\mathrm{d}}{\mathrm{d} x} P^{n+1}=0 .
\end{aligned}
$$

Following a similar procedure as for the $\theta$ method we arrive to

$$
\omega_{\delta t} \delta t=\frac{1}{\mathrm{i}} \log (2 \pm \sqrt{1-2 \mathrm{i} \omega \delta t}) .
$$

The numerical angular frequency is complex. The root corresponding to $+\sqrt{ }$ is spurious, hence we just plot the root corresponding to $-\sqrt{ }$. It can be shown that the angular frequency error is 2 nd order in $\omega \delta t$. 

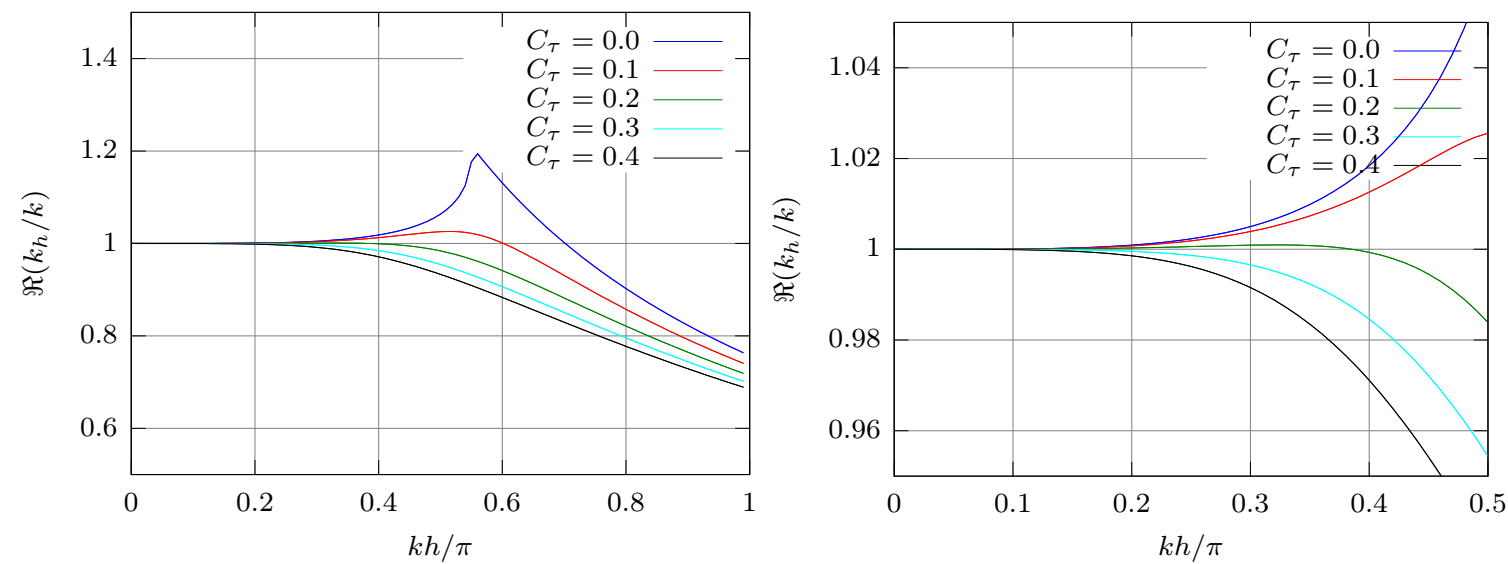

Figure 4: ASGS space semi-discretization: real part of the wavenumber ratio (the right picture is a zoom of the left)
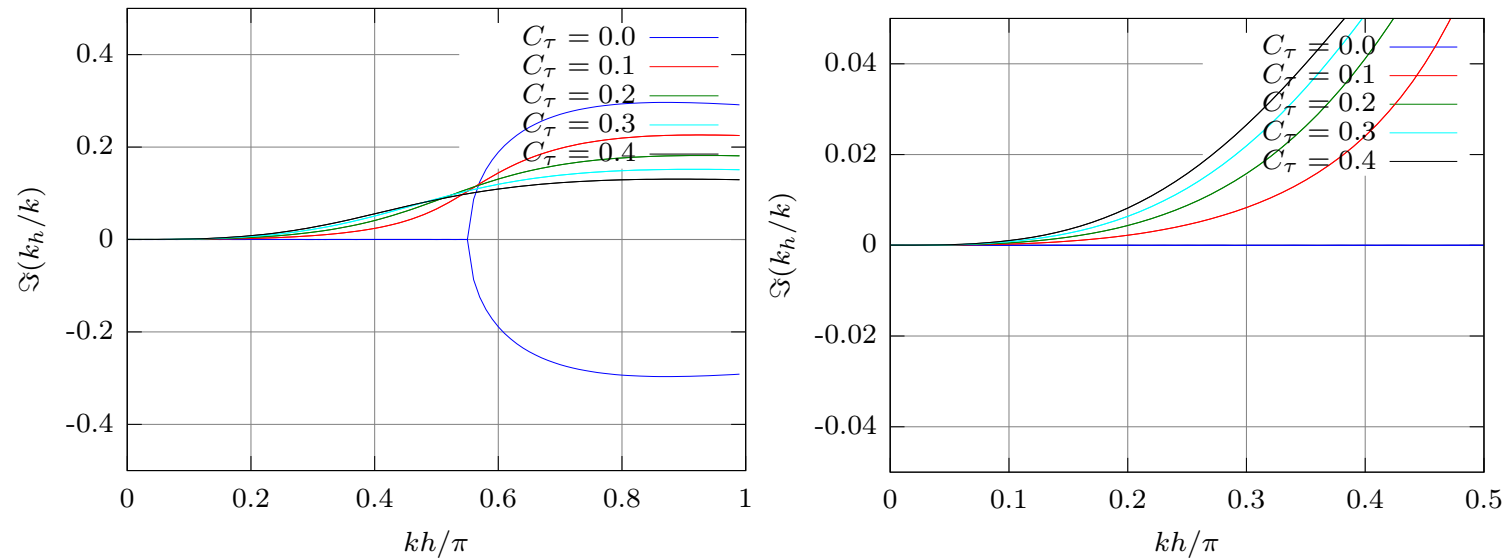

Figure 5: ASGS space semi-discretization: imaginary part of the wavenumber ratio (the right picture is a zoom of the left)

Fig. 2 shows the angular frequency ratio $\omega_{\delta t} / \omega$ for $0 \leq \omega \delta t / \pi \leq 1$. It can be seen that the BDF2 method is unconditionally stable.

Now, let us compare the $\theta$-method with $\theta=1 / 2$ and BDF2. Fig. 3 compares both methods. It can be seen that the $\theta$-method with $\theta=1 / 2$ outperforms BDF2 for the wave equation in mixed form both in terms of dispersion and dissipation.

\subsection{Space semi-discretization}

We analyze now the wave equation in mixed form when the spatial discretization is done using the ASGS and the OSS methods. We take $\omega_{h}=k_{h} c_{h}$ and $\omega_{h}=\omega$, where the subscript $h$ denotes spatial semi-discretization. We take now

$$
\left[P_{j}(t), U_{j}(t)\right]=\phi_{\omega} \mathrm{e}^{\mathrm{i}\left(k_{h} x_{j}-\omega t\right)}\left[1, \mu_{p}^{1 / 2} \mu_{u}^{-1 / 2}\right],
$$

where $\phi_{\omega}$ is a constant that describes the amplitude at $t=0$.

For the ASGS method, the semi-discrete problem has the matrix structure:

$$
\begin{aligned}
& \mu_{p} M_{p} \frac{\mathrm{d} P}{\mathrm{~d} t}+K_{u} U+\tau_{u} \mu_{u} M_{S, u} \frac{\mathrm{d} U}{\mathrm{~d} t}+\tau_{u} K_{S, p} P=0, \\
& \mu_{u} M_{u} \frac{\mathrm{d} U}{\mathrm{~d} t}+K_{p} P+\tau_{p} \mu_{p} M_{S, p} \frac{\mathrm{d} P}{\mathrm{~d} t}+\tau_{p} K_{S, u} U=0,
\end{aligned}
$$



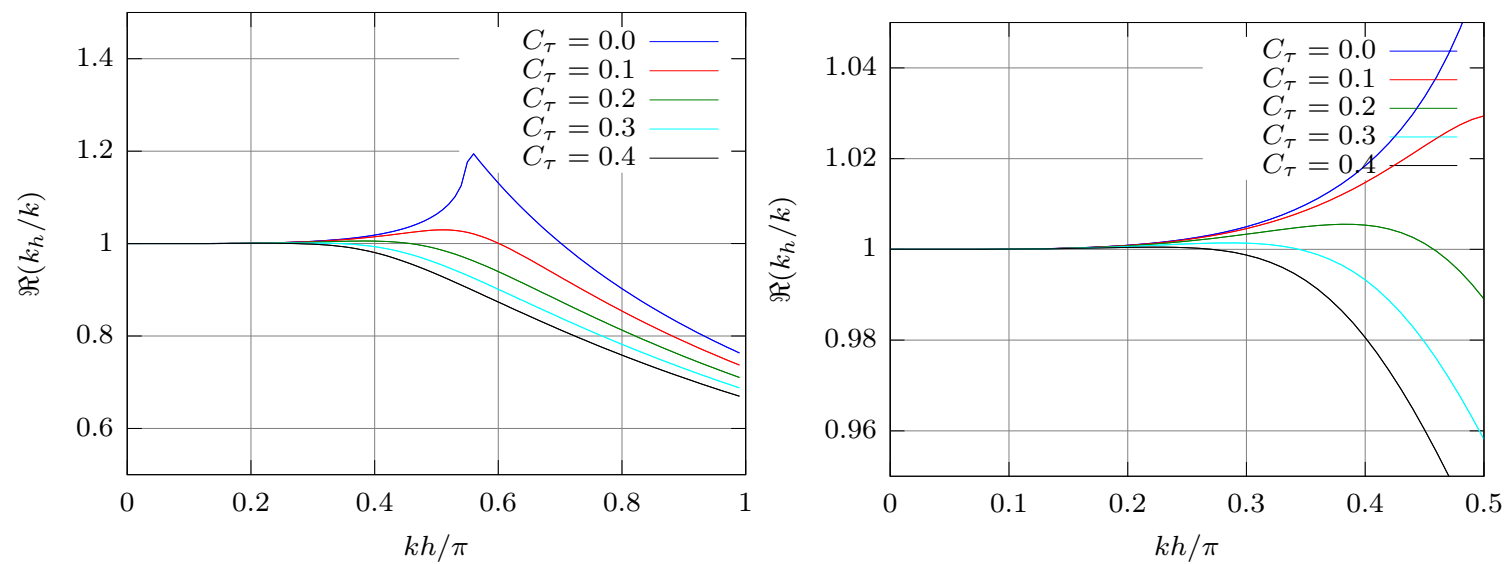

Figure 6: OSS space semi-discretization: real part of the wavenumber ratio (the right picture is a zoom of the left)
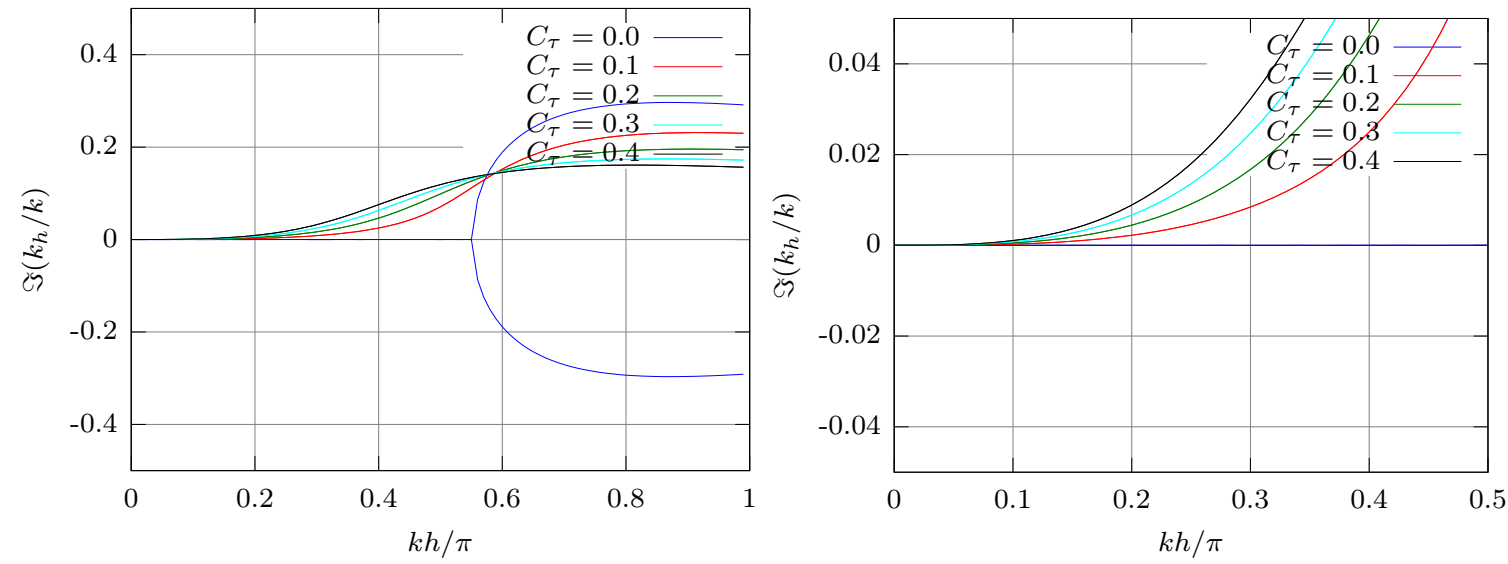

Figure 7: OSS space semi-discretization: imaginary part of the wavenumber ratio (the right picture is a zoom of the left) 
where a subscript $S$ has been introduced in the matrices with contributions from the stabilization terms. In these equations, $P$ and $U$ do not denote the sequence of solutions in time, but the array of nodal unknowns with time-continuous components. The meaning of $P$ and $U$ in what follows will be determined by the context. We just analyze one of the previous equations because they are equivalent. The $j$-th row of the first system of equations is

$$
\begin{aligned}
\mu_{p} \frac{h}{6} & \left(\frac{\mathrm{d}}{\mathrm{d} t} P_{j-1}+4 \frac{\mathrm{d}}{\mathrm{d} t} P_{j}+\frac{\mathrm{d}}{\mathrm{d} t} P_{j+1}\right)+\frac{1}{2}\left(-U_{j-1}+U_{j+1}\right) \\
& +\tau_{u} \mu_{u} \frac{1}{2}\left(\frac{\mathrm{d}}{\mathrm{d} t} U_{j-1}-\frac{\mathrm{d}}{\mathrm{d} t} U_{j+1}\right)+\tau_{u} \frac{1}{h}\left(-P_{j-1}+2 P_{j}-P_{j+1}\right)=0 .
\end{aligned}
$$

Replacing $\left[P_{j}, U_{j}\right]$ from 43$]$ we have:

$$
\begin{aligned}
-\mathrm{i} \omega \mu_{p} & \frac{h}{6}\left(\mathrm{e}^{-\mathrm{i} k_{h} h}+4+\mathrm{e}^{\mathrm{i} k_{h} h}\right)+\frac{1}{2} \mu_{p}^{1 / 2} \mu_{u}^{-1 / 2}\left(-\mathrm{e}^{-\mathrm{i} k_{h} h}+\mathrm{e}^{\mathrm{i} k_{h} h}\right) \\
- & \frac{1}{2} \mathrm{i} \omega \tau_{u} \mu_{p}^{1 / 2} \mu_{u}^{1 / 2}\left(\mathrm{e}^{-\mathrm{i} k_{h} h}-\mathrm{e}^{\mathrm{i} k_{h} h}\right)+\frac{1}{h} \tau_{u}\left(-\mathrm{e}^{-\mathrm{i} k_{h} h}+2-\mathrm{e}^{\mathrm{i} k_{h} h}\right)=0, \\
\frac{k_{h}}{k} & =-\frac{\mathrm{i}}{k h} \log \left(\frac{-\left(4 k h+12 \mathrm{i} C_{\tau}\right) \pm \sqrt{36(k h)^{2} C_{\tau}^{2}+72 \mathrm{i} k h C_{\tau}+12(k h)^{2}-36}}{2\left(k h+3 \mathrm{i}-3 k h C_{\tau}-6 \mathrm{i} C_{\tau}\right)}\right) .
\end{aligned}
$$

The Maclaurin series for the real and imaginary parts are:

$$
\begin{aligned}
& \frac{\Re\left(k_{h}\right)}{k}=1-\frac{15 C_{\tau}^{2}-1}{180}(k h)^{4}+\frac{126 C_{\tau}^{4}-63 C_{\tau}^{2}+1}{1512}(k h)^{6}+\ldots, \\
& \frac{\Im\left(k_{h}\right)}{k}=\frac{C_{\tau}}{12}(k h)^{3}-\frac{6 C_{\tau}^{3}-C_{\tau}}{72}(k h)^{5}+\ldots .
\end{aligned}
$$

It can be shown that $k_{h}$ is complex and that $\Re\left(k_{h}\right)$ is of order $(k h)^{4}$ for any $C_{\tau}$ and of order $(k h)^{6}$ for $C_{\tau}=1 / \sqrt{15} \approx 0.2582$. Additionally, $\Im\left(k_{h}\right)$ is of order $(k h)^{3}$ for any $C_{\tau}>0$. Fig. 4 and Fig. 5 show the real and imaginary parts of the wavenumber ratio as a function of $k h / \pi$. It can be seen that the Galerkin method $\left(C_{\tau}=0\right)$ is unstable whereas the ASGS method is always stable.

Next, we analyze an OSS method in which a lumped mass matrix (diagonal) is used to project the residual. Additionally, as the projection with a lumped mass matrix is not exactly an $L^{2}$ projection, we keep the time derivatives in the residual. Non-lumped (consistent) mass matrices are used for time derivatives. Better than lumped mass matrix approximations to the $L^{2}$ projection can be used, but the results obtained are very similar. For instance we could use the family of banded approximate mass matrices from [31.

The matrix form of the semi-discrete problem is:

$$
\begin{array}{r}
\mu_{p} M_{p} \frac{\mathrm{d} P}{\mathrm{~d} t}+K_{u} U+\tau_{u} \mu_{u} M_{S, u} \frac{\mathrm{d} U}{\mathrm{~d} t}+\tau_{u} K_{S, p} P-\tau_{u} M_{S, u} R_{u}=0 \\
M_{u} R_{u}=\mu_{u} M_{u} \frac{\mathrm{d}}{\mathrm{d} t} U+K_{p} P \\
\mu_{u} M_{u} \frac{\mathrm{d} U}{\mathrm{~d} t}+K_{p} P+\tau_{p} \mu_{p} M_{S, p} \frac{\mathrm{d} P}{\mathrm{~d} t}+\tau_{p} K_{S, u} U-\tau_{p} M_{S, p} R_{p}=0 \\
M_{p} R_{p}=\mu_{p} M_{p} \frac{\mathrm{d}}{\mathrm{d} t} P+K_{u} U
\end{array}
$$

As we use a lumped projection, the semi-discrete problem reduces to:

$$
\begin{aligned}
& \mu_{p} M_{p} \frac{\mathrm{d} P}{\mathrm{~d} t}+K_{u} U+\tau_{u} \mu_{u} M_{S, u} \frac{\mathrm{d} U}{\mathrm{~d} t}+\tau_{u} K_{S, p} P-\tau_{u} M_{S, u} \widetilde{M}_{u}^{-1}\left(\mu_{u} M_{u} \frac{\mathrm{d}}{\mathrm{d} t} U+K_{p} P\right)=0 \\
& \mu_{u} M_{u} \frac{\mathrm{d} U}{\mathrm{~d} t}+K_{p} P+\tau_{p} \mu_{p} M_{S, p} \frac{\mathrm{d} P}{\mathrm{~d} t}+\tau_{p} K_{S, u} U-\tau_{p} M_{S, p} \widetilde{M}_{p}^{-1}\left(\mu_{p} M_{p} \frac{\mathrm{d}}{\mathrm{d} t} P+K_{u} U\right)=0 .
\end{aligned}
$$



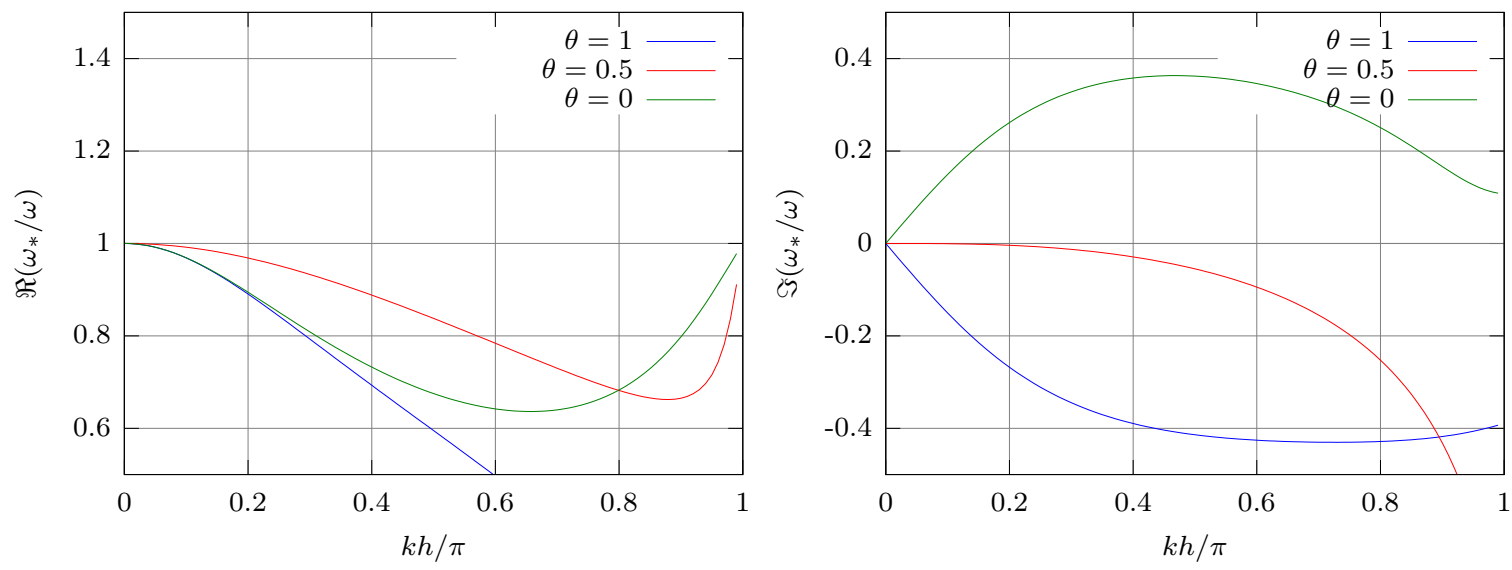

Figure 8: ASGS $+\theta$ for $r=1$ and $C_{\tau}=0.2$
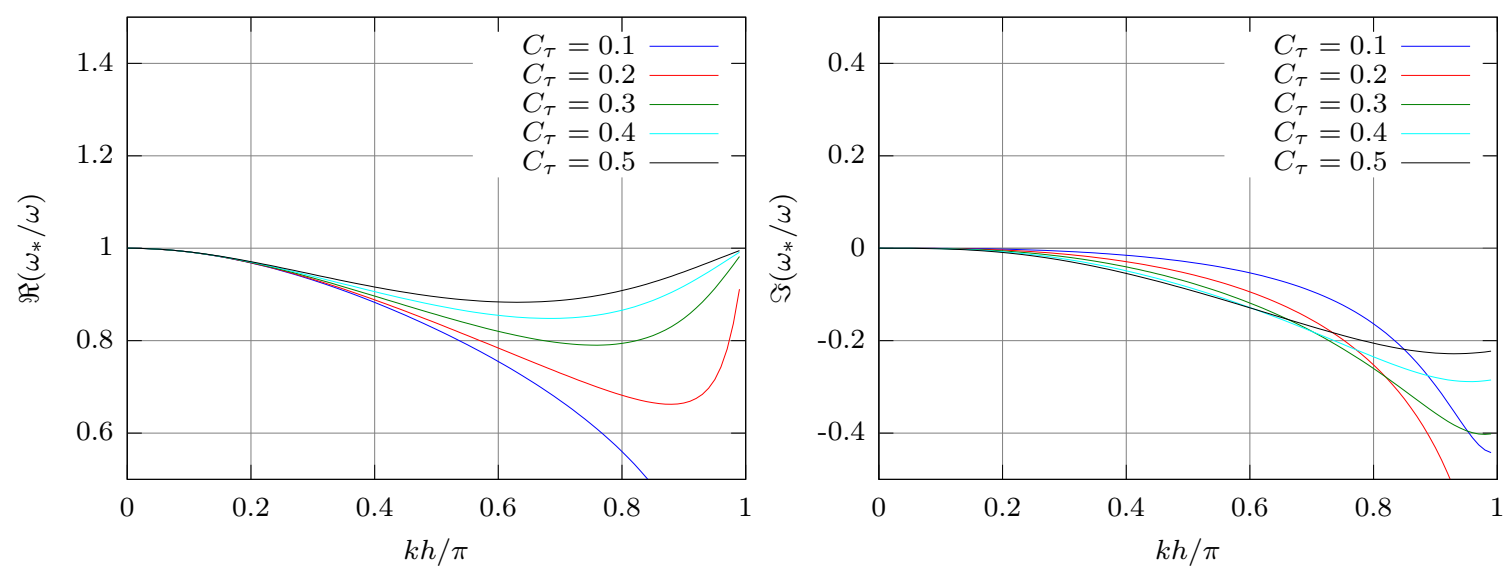

Figure 9: ASGS $+\theta$ for $r=1$ and $\theta=0.5$

Following similar steps as for the ASGS method, we arrive to the expression which relates $k_{h} h$ with $k h$ :

$$
\begin{aligned}
& 2 k h\left(\mathrm{e}^{\mathrm{i} k_{h} h}+4 \mathrm{e}^{2 \mathrm{i} k_{h} h}+\mathrm{e}^{3 \mathrm{i} k_{h} h}\right)+6 \mathrm{i}\left(-\mathrm{e}^{\mathrm{i} k_{h} h}+\mathrm{e}^{3 \mathrm{i} k_{h} h}\right) \\
& \quad+k h C_{\tau}\left(-1+2 \mathrm{e}^{\mathrm{i} k_{h} h}-2 \mathrm{e}^{3 \mathrm{i} k_{h} h}+\mathrm{e}^{4 \mathrm{i} k_{h} h}\right)+3 \mathrm{i} C_{\tau}\left(1-4 \mathrm{e}^{\mathrm{i} k_{h} h}+6 \mathrm{e}^{2 \mathrm{i} k_{h} h}-4 \mathrm{e}^{3 \mathrm{i} k_{h} h}+\mathrm{e}^{4 \mathrm{i} k_{h} h}\right)=0 .
\end{aligned}
$$

Fig. 6 and Fig. 7 show the real and imaginary parts of the wavenumber ratio as a function of $k h / \pi$. As before, the Galerkin method $\left(C_{\tau}=0\right)$ is unstable whereas the OSS method is always stable.

\subsection{Space-time discretization}

Finally, we analyze the fully discrete problems arising from the combination of time discretizations $(\theta$ and BDF2) and space discretizations (ASGS and OSS). Thus, in total we will analyze four fully discrete problems. We take $\omega_{*}=k_{*} c_{*}$ and $k_{*}=k$. The subscript $*$ denotes full discretization. We take a mode of the form

$$
\left[P_{j}^{n}, U_{j}^{n}\right]=\phi_{k} \mathrm{e}^{\mathrm{i}\left(k x_{j}-\omega_{*} t_{n}\right)}\left[1, \mu_{p}^{1 / 2} \mu_{u}^{-1 / 2}\right],
$$

where $\phi_{k}$ describes the amplitude at $t_{n}=0$.

Let us define $r$ as the Courant or CFL number as

$$
r:=c \delta t / h \text {. }
$$



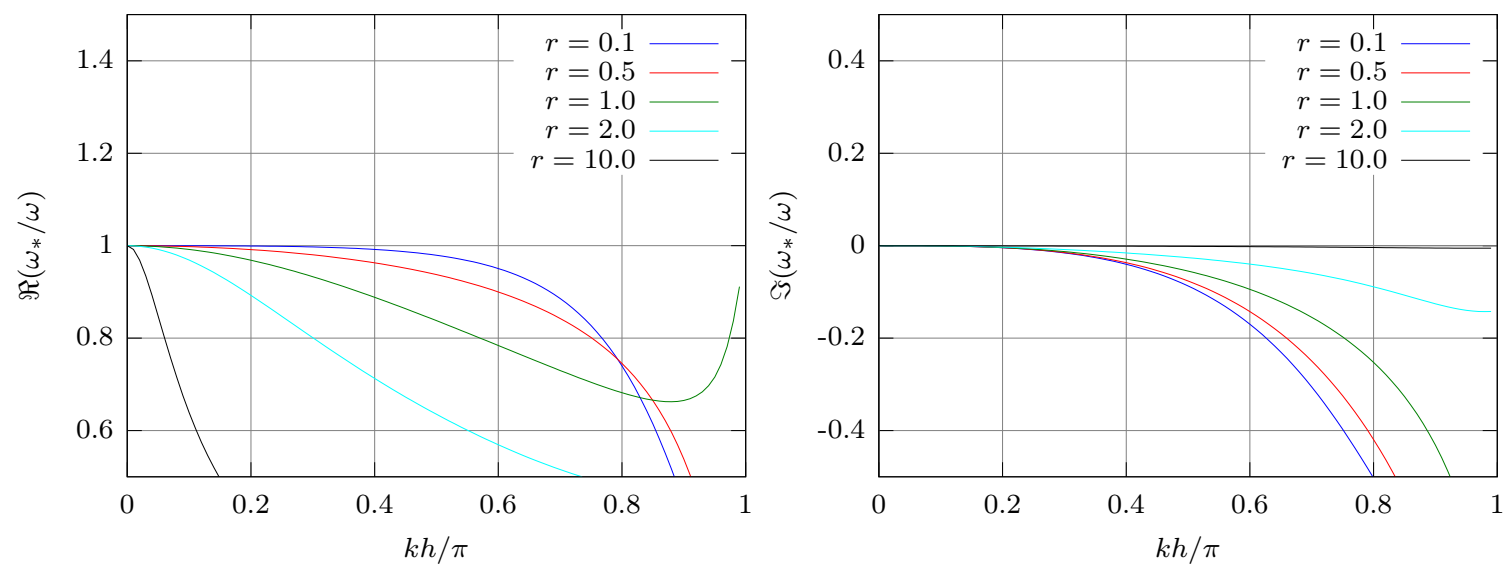

Figure 10: ASGS $+\theta$ for $C_{\tau}=0.2$ and $\theta=0.5$
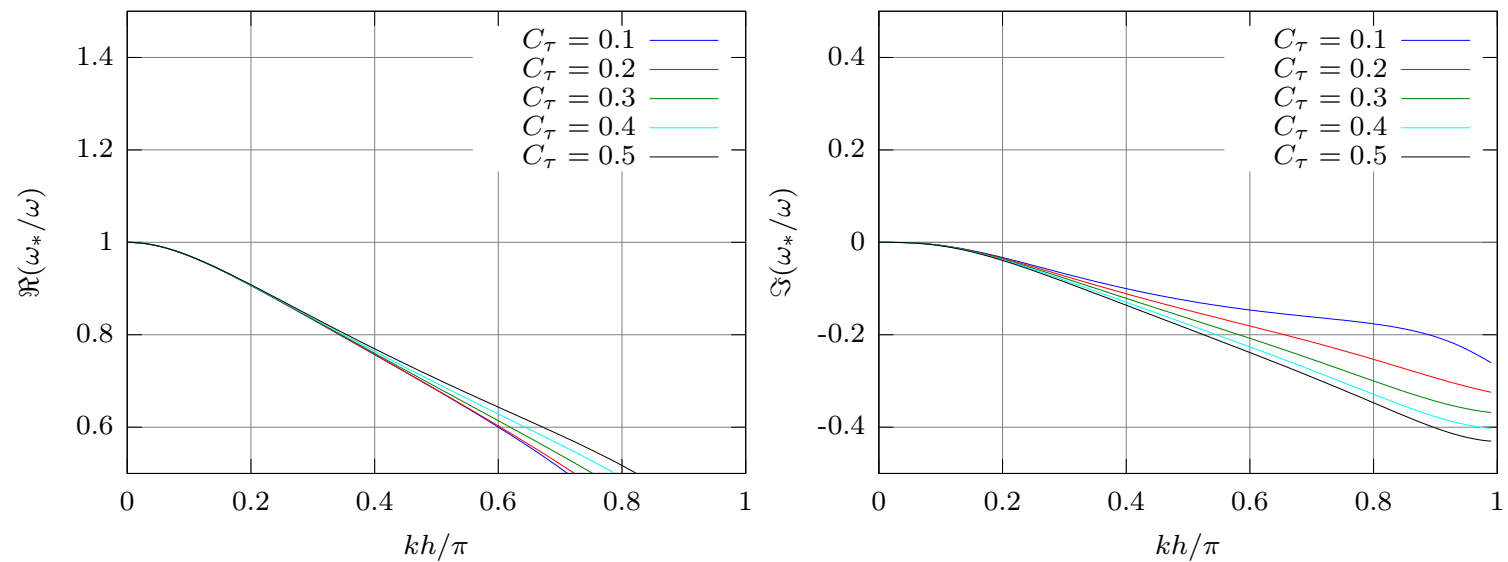

Figure 11: ASGS + BDF2 for $r=1$
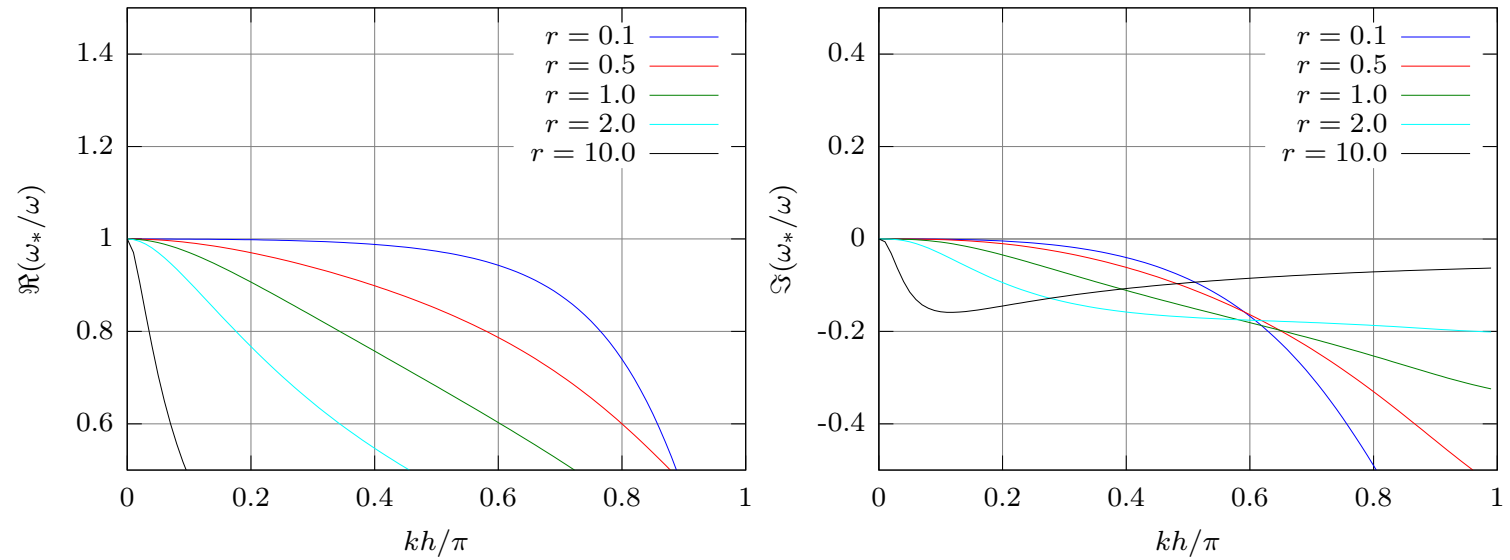

Figure 12: ASGS + BDF2 for $C_{\tau}=0.2$ 
First, we consider the ASGS formulation for the space discretization. With regard to the time integration, we first consider the $\theta$-method. The fully discrete problem is:

$$
\begin{aligned}
\frac{\mu_{p}}{\delta t} M_{p}\left(P^{n+1}-P^{n}\right)+ & \tau_{u} \frac{\mu_{u}}{\delta t} M_{S, u}\left(U^{n+1}-U^{n}\right)+\theta\left(K_{u} U^{n+1}+\tau_{u} K_{S, p} P^{n+1}\right) \\
& +(1-\theta)\left(K_{u} U^{n}+\tau_{u} K_{S, p} P^{n}\right)=0 \\
\frac{\mu_{u}}{\delta t} M_{u}\left(U^{n+1}-U^{n}\right)+ & \tau_{p} \frac{\mu_{p}}{\delta t} M_{S, p}\left(P^{n+1}-P^{n}\right)+\theta\left(K_{p} P^{n+1}+\tau_{p} K_{S, u} U^{n+1}\right) \\
+ & (1-\theta)\left(K_{p} P^{n}+\tau_{p} K_{S, u} U^{n}\right)=0 .
\end{aligned}
$$

We concentrate in one of the equations because both will be equivalent for the current analysis. The $j$-th row of the system of equations is

$$
\begin{aligned}
\mu_{p} \frac{h}{6 \delta t} & \left(P_{j-1}^{n+1}+4 P_{j}^{n+1}+P_{j+1}^{n+1}-P_{j-1}^{n}-4 P_{j}^{n}-P_{j+1}^{n}\right)+\tau_{u} \frac{\mu_{u}}{2 \delta t}\left(U_{j-1}^{n+1}-U_{j+1}^{n+1}-U_{j-1}^{n}+U_{j+1}^{n}\right) \\
& +\theta\left(\frac{1}{2}\left(-U_{j-1}^{n+1}+U_{j+1}^{n+1}\right)+\tau_{u} \frac{1}{h}\left(-P_{j-1}^{n+1}+2 P_{j}^{n+1}-P_{j+1}^{n+1}\right)\right) \\
& +(1-\theta)\left(\frac{1}{2}\left(-U_{j-1}^{n}+U_{j+1}^{n}\right)+\tau_{u} \frac{1}{h}\left(-P_{j-1}^{n}+2 P_{j}^{n}-P_{j+1}^{n}\right)\right)=0,
\end{aligned}
$$

and replacing $\left[P_{j}^{n}, U_{j}^{n}\right]$ from 44 we get

$$
\begin{aligned}
& \mu_{p} \frac{h}{6 \delta t}\left(\mathrm{e}^{\mathrm{i}\left(-k h-\omega_{*} \delta t\right)}+4 \mathrm{e}^{\mathrm{i}\left(-\omega_{*} \delta t\right)}+\mathrm{e}^{\mathrm{i}\left(k h-\omega_{*} \delta t\right)}-\mathrm{e}^{\mathrm{i}(-k h)}-4-\mathrm{e}^{\mathrm{i}(k h)}\right) \\
& +\frac{1}{2 \delta t} \tau_{u} \mu_{p}^{1 / 2} \mu_{u}^{1 / 2}\left(\mathrm{e}^{\mathrm{i}\left(-k h-\omega_{*} \delta t\right)}-\mathrm{e}^{\mathrm{i}\left(k h-\omega_{*} \delta t\right)}-\mathrm{e}^{\mathrm{i}(-k h)}+\mathrm{e}^{\mathrm{i}(k h)}\right) \\
& +\theta\left(\frac{1}{2} \mu_{p}^{1 / 2} \mu_{u}^{-1 / 2}\left(-\mathrm{e}^{\mathrm{i}\left(-k h-\omega_{*} \delta t\right)}+\mathrm{e}^{\mathrm{i}\left(k h-\omega_{*} \delta t\right)}\right)+\frac{1}{h} \tau_{u}\left(-\mathrm{e}^{\mathrm{i}\left(-k h-\omega_{*} \delta t\right)}+2 \mathrm{e}^{\mathrm{i}\left(-\omega_{*} \delta t\right)}-\mathrm{e}^{\mathrm{i}\left(k h-\omega_{*} \delta t\right)}\right)\right) \\
& +(1-\theta)\left(\frac{1}{2} \mu_{p}^{1 / 2} \mu_{u}^{-1 / 2}\left(-\mathrm{e}^{\mathrm{i}(-k h)}+\mathrm{e}^{\mathrm{i}(k h)}\right)+\frac{1}{h} \tau_{u}\left(-\mathrm{e}^{\mathrm{i}(-k h)}+2-\mathrm{e}^{\mathrm{i}(k h)}\right)\right)=0 .
\end{aligned}
$$

Using the definition of $r$ from 45 we arrive to:

$$
\frac{\omega_{*}}{\omega}=-\frac{1}{\mathrm{i} r(k h)} \log \left(\frac{-2-\cos (k h)+3 \mathrm{i} C_{\tau} \sin (k h)+(1-\theta)\left(3 \mathrm{i} r \sin (k h)+6 r C_{\tau}(1-\cos (k h))\right)}{3 \mathrm{i} C_{\tau} \sin (k h)-2-\cos (k h)-3 \mathrm{i} r \theta \sin (k h)-6 r \theta C_{\tau}(1-\cos (k h))}\right) .
$$

Fig. 8, Fig. 9, and Fig. 10 show the real and imaginary parts of the angular frequency ratio keeping two parameters fixed. It can be seen that $\theta=0$ (forward Euler or explicit Euler scheme) is unconditionally unstable. This is similar to what happens with forward in time-centered in space finite differences (FTCS) applied to the pure advection equation in 1D.

Next, we consider the ASGS with the BDF2 time integration. The fully discrete problem is:

$$
\begin{aligned}
& \frac{\mu_{p}}{2 \delta t} M_{p}\left(3 P^{n+2}-4 P^{n+1}+P^{n}\right)+\tau_{u} \frac{\mu_{u}}{2 \delta t} M_{S, u}\left(3 U^{n+2}-4 U^{n+1}+U^{n}\right)+K_{u} U^{n+2}+\tau_{u} K_{S, p} P^{n+2}=0 \\
& \frac{\mu_{u}}{2 \delta t} M_{u}\left(3 U^{n+2}-4 U^{n+1}+U^{n}\right)+\tau_{p} \frac{\mu_{p}}{2 \delta t} M_{S, p}\left(3 P^{n+2}-4 P^{n+1}+P^{n}\right)+K_{p} P^{n+2}+\tau_{p} K_{S, u} U^{n+2}=0 .
\end{aligned}
$$

Following a similar procedure as before, we arrive to

$$
\begin{array}{r}
\left(3 \mathrm{e}^{-2 \mathrm{i} \omega_{*} \delta t}-4 \mathrm{e}^{-\mathrm{i} \omega_{*} \delta t}+1\right)\left((2+\cos (k h))-3 \mathrm{i} C_{\tau}(\sin (k h))\right) \\
+\mathrm{e}^{-2 \mathrm{i} \omega_{*} \delta t}\left(6 \mathrm{i} r(\sin (k h))+12 r C_{\tau}(1-\cos (k h))\right)=0 .
\end{array}
$$

Fig. 11 and Fig. 12 show the angular frequency ratio for the fully discrete problem. It can be seen that this combination of spatial and temporal discretization is stable. 

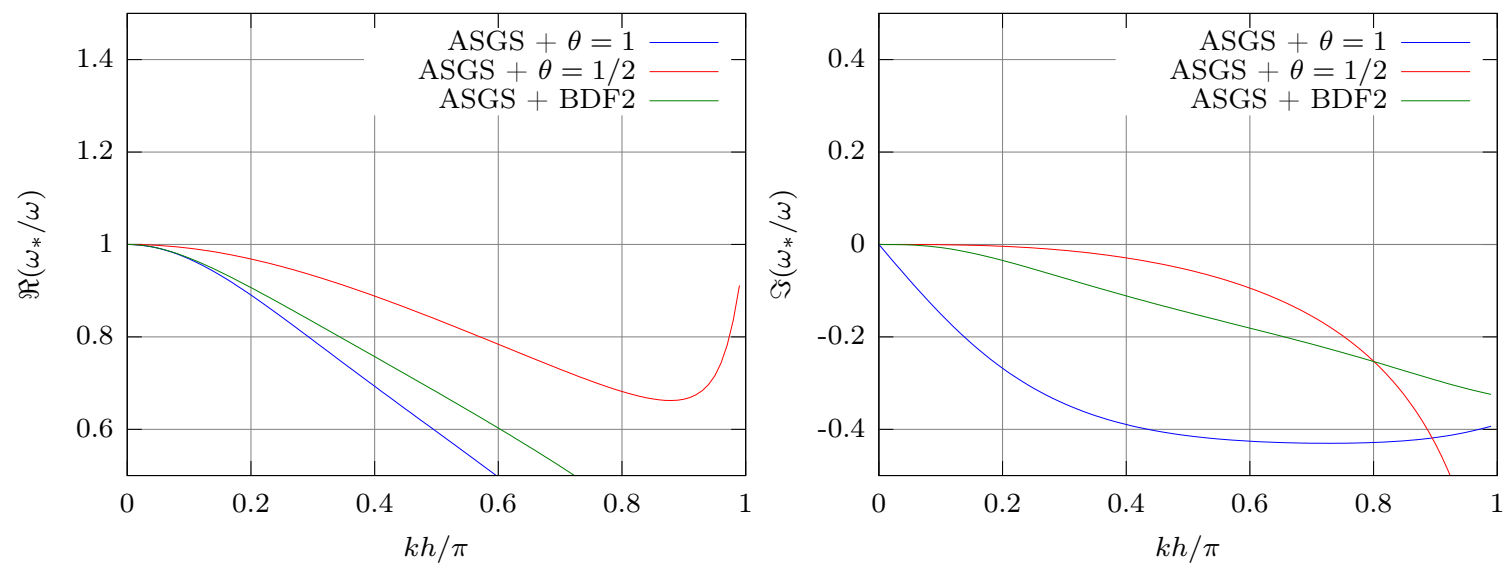

Figure 13: Fully discrete ASGS with $C_{\tau}=0.2$ and $r=1$

Fig. 13 compares the fully discrete ASGS method with the two time integration schemes shown previously. It can be seen that the $\theta$ method performs better than BDF2 for small enough $k h$.

Next, we aim to analyze the properties of the OSS stabilized formulation. When using the $\theta$-method for the time integration, the fully discrete problem is:

$$
\begin{aligned}
& \frac{\mu_{p}}{\delta t} M_{p}\left(P^{n+1}-P^{n}\right)+\tau_{u} \frac{\mu_{u}}{\delta t} M_{S, u}\left(U^{n+1}-U^{n}\right)-\tau_{u} M_{S, u} \widetilde{M}_{u}^{-1} \frac{\mu_{u}}{\delta t} M_{u}\left(U^{n+1}-U^{n}\right) \\
& \quad+\theta\left(K_{u} U^{n+1}+\tau_{u} K_{S, p} P^{n+1}-\tau_{u} M_{S, u} \widetilde{M}_{u}^{-1} K_{p} P^{n+1}\right) \\
& \quad+(1-\theta)\left(K_{u} U^{n}+\tau_{u} K_{S, p} P^{n}-\tau_{u} M_{S, u} \widetilde{M}_{u}^{-1} K_{p} P^{n}\right)=0, \\
& \frac{\mu_{u}}{\delta t} M_{u}\left(U^{n+1}-U^{n}\right)+\tau_{p} \frac{\mu_{p}}{\delta t} M_{S, p}\left(P^{n+1}-P^{n}\right)-\tau_{p} M_{S, p} \widetilde{M}_{p}^{-1} \frac{\mu_{p}}{\delta t} M_{p}\left(P^{n+1}-P^{n}\right) \\
& \quad+\theta\left(K_{p} P^{n+1}+\tau_{p} K_{S, u} U^{n+1}-\tau_{p} M_{S, p} \widetilde{M}_{p}^{-1} K_{u} U^{n+1}\right) \\
& \quad+(1-\theta)\left(K_{p} P^{n}+\tau_{p} K_{S, u} U^{n}-\tau_{p} M_{S, p} \widetilde{M}_{p}^{-1} K_{u} U^{n}\right)=0 .
\end{aligned}
$$

Following a similar procedure as before we arrive to

$$
\begin{aligned}
& \left(\mathrm{e}^{-\mathrm{i} \omega_{*} \delta t}-1\right)\left(2(2+\cos (k h))+\mathrm{i} C_{\tau}(-2 \sin (k h)+\sin (2 k h))\right) \\
& +\left(\theta \mathrm{e}^{-\mathrm{i} \omega_{*} \delta t}+1-\theta\right)\left(6 \mathrm{i} r(\sin (k h))+3 r C_{\tau}(3-4 \cos (k h)+\cos (2 k h))\right)=0 .
\end{aligned}
$$

Fig. 14, Fig. 15, and Fig. 16 show the angular frequency ratio for this fully discretized problem.

Next, we consider the OSS-BDF2 fully discrete problem:

$$
\begin{aligned}
& \frac{\mu_{p}}{2 \delta t} M_{p}\left(3 P^{n+2}-4 P^{n+1}+P^{n}\right)+\tau_{u} \frac{\mu_{u}}{2 \delta t} M_{S, u}\left(3 U^{n+2}-4 U^{n+1}+U^{n}\right)+K_{u} U^{n+2}+\tau_{u} K_{S, p} P^{n+2} \\
& \quad-\tau_{u} M_{S, u} \widetilde{M}_{u}^{-1}\left(\frac{\mu_{u}}{2 \delta t} M_{u}\left(3 U^{n+2}-4 U^{n+1}+U^{n}\right)+K_{p} P^{n+2}\right)=0 \\
& \frac{\mu_{u}}{2 \delta t} M_{u}\left(3 U^{n+2}-4 U^{n+1}+U^{n}\right)+\tau_{p} \frac{\mu_{p}}{2 \delta t} M_{S, p}\left(3 P^{n+2}-4 P^{n+1}+P^{n}\right)+K_{p} P^{n+2}+\tau_{p} K_{S, u} U^{n+2} \\
& \quad-\tau_{p} M_{S, p} \widetilde{M}_{p}^{-1}\left(\frac{\mu_{p}}{2 \delta t} M_{p}\left(3 P^{n+2}-4 P^{n+1}+P^{n}\right)+K_{u} U^{n+2}\right)=0 .
\end{aligned}
$$

Following a similar procedure as before we get

$$
\begin{aligned}
& \left(3 \mathrm{e}^{-2 \mathrm{i} \omega_{*} \delta t}-4 \mathrm{e}^{-\mathrm{i} \omega_{*} \delta t}+1\right)\left(2(2+\cos (k h))+\mathrm{i} C_{\tau}(-2 \sin (k h)+\sin (2 k h))\right) \\
& +\mathrm{e}^{-2 \mathrm{i} \omega_{*} \delta t}\left(12 \mathrm{i} r(\sin (k h))+6 r C_{\tau}(3-4 \cos (k h)+\cos (2 k h))\right)=0 .
\end{aligned}
$$



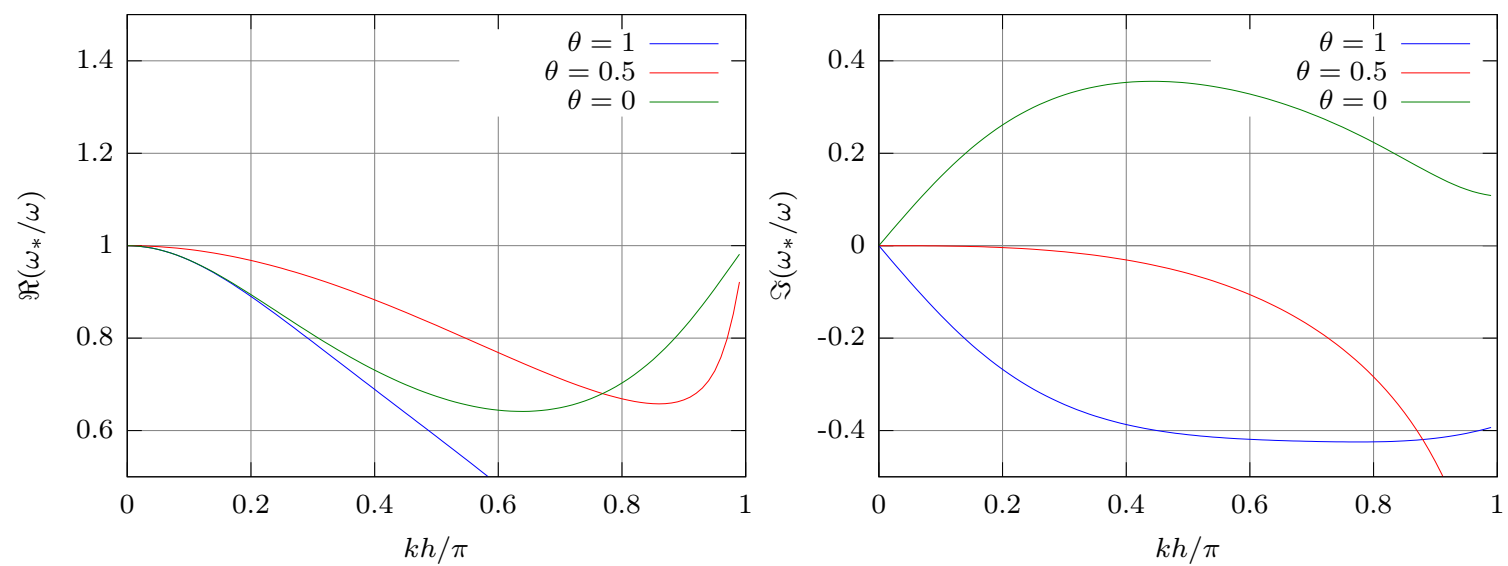

Figure 14: OSS $+\theta$ for $r=1$ and $C_{\tau}=0.2$
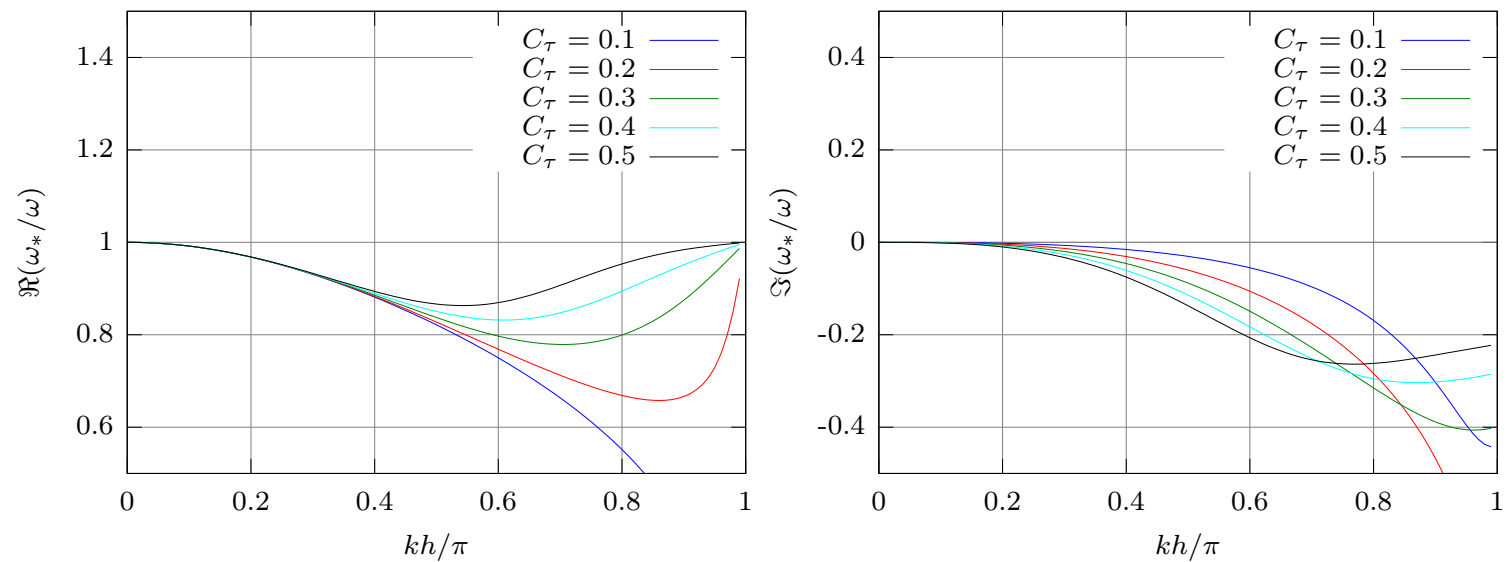

Figure 15: OSS $+\theta$ for $r=1$ and $\theta=0.5$
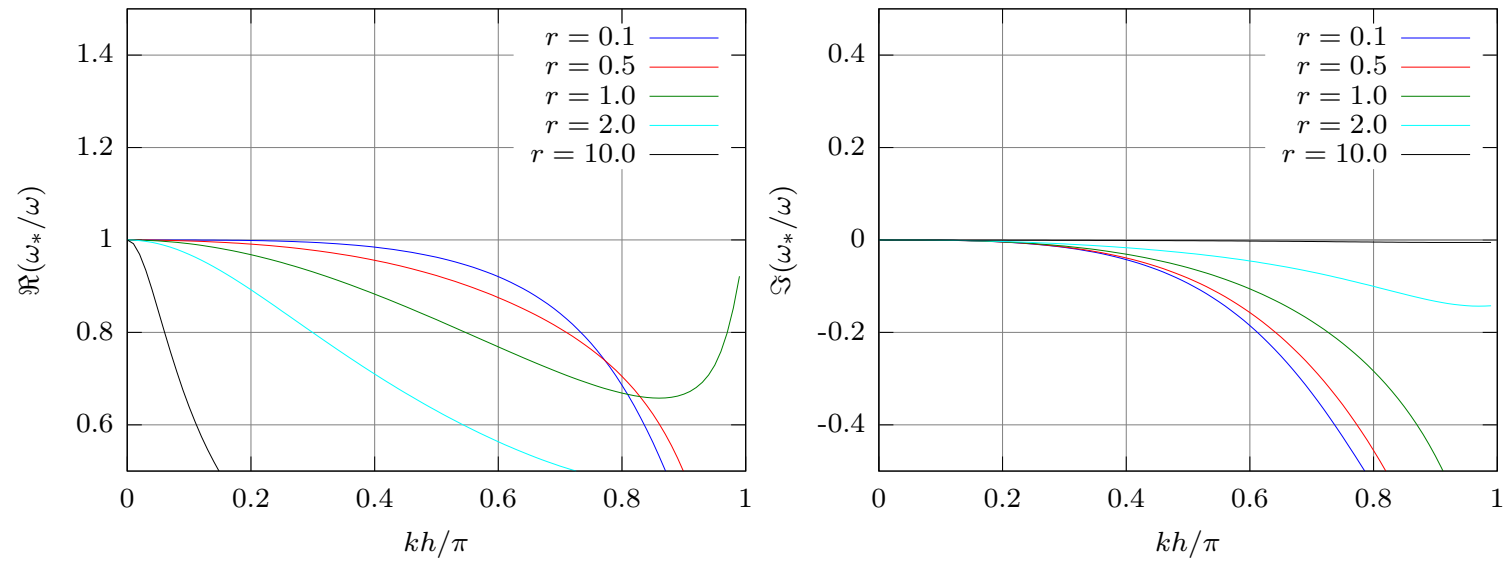

Figure 16: OSS $+\theta$ for $C_{\tau}=0.2$ and $\theta=0.5$ 

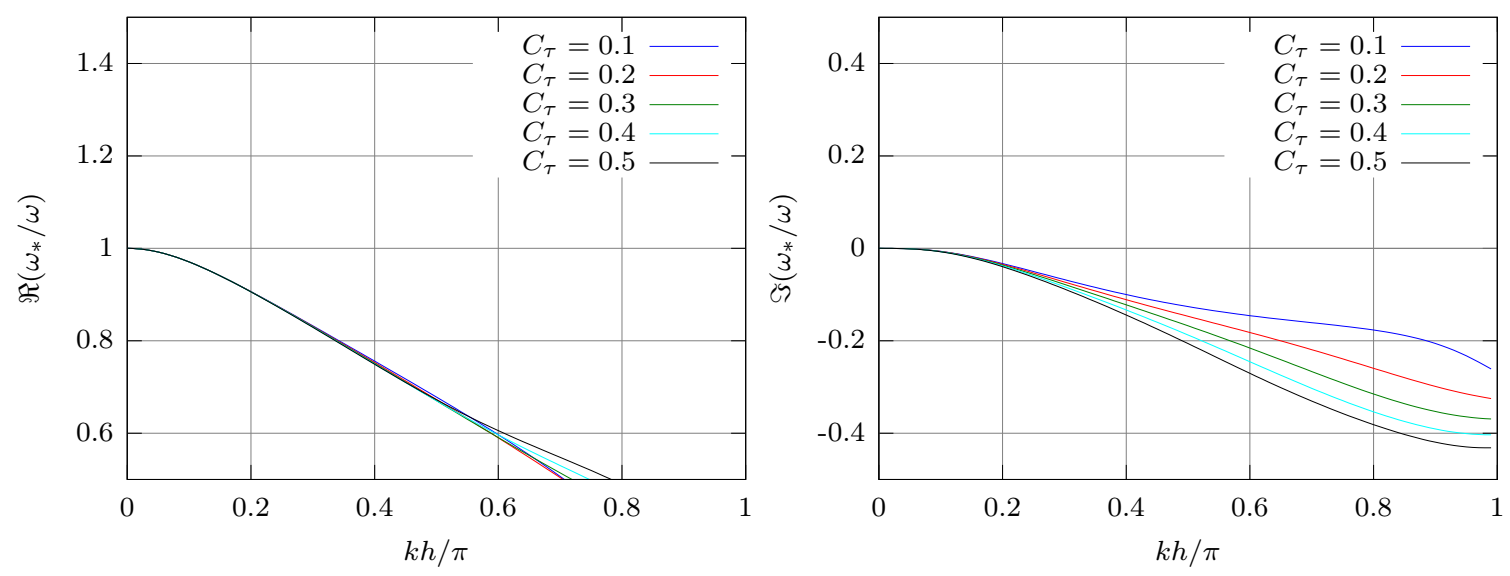

Figure 17: OSS + BDF2 for $r=1$
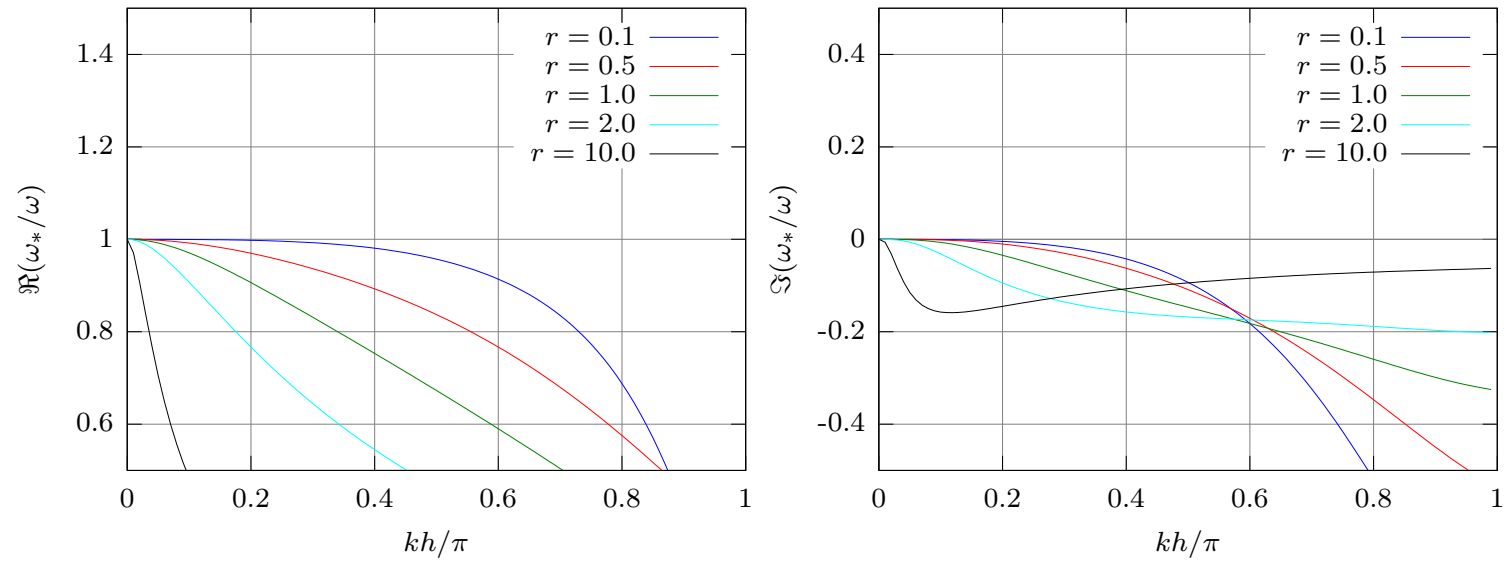

Figure 18: OSS + BDF2 for $C_{\tau}=0.2$ 
Table 9: Experimental $\delta t$ convergence rates for ASGS-BE method using $Q 1 / Q 1$ spatial interpolation

\begin{tabular}{|c|c|c|c|c|c|c|c|c|}
\hline Variational Form & \multicolumn{2}{|c|}{ I } & \multicolumn{2}{|c|}{ II } & \multicolumn{2}{|c|}{ III } & \multicolumn{2}{|c|}{$\mathrm{I}$} \\
\hline$\delta t$ vs. $h$ & & & $\delta t \sim$ & $.5 h$ & & & $\delta t \sim 2$ & $h^{1.5}$ \\
\hline & Num & Min & Num & Min & Num & Min & Num & Min \\
\hline$\left\|p^{n}-p_{h}^{n}\right\|_{\ell^{\infty}\left(L^{2}\right)}$ & 1.00 & 1 & 1.00 & 1 & 1.01 & 1 & 1.00 & 1 \\
\hline$\left\|\boldsymbol{u}^{n}-\boldsymbol{u}_{h}^{n}\right\|_{\ell^{\infty}\left(L^{2}\right)}$ & 1.02 & 1 & 1.02 & 1 & 1.02 & 1 & 1.01 & 1 \\
\hline$\left\|\nabla\left(p^{n}-p_{h}^{n}\right)\right\|_{\ell^{2}\left(L^{2}\right)}$ & 1.00 & 0.5 & 1.00 & 0 & 1.00 & 1 & 0.66 & $2 / 3$ \\
\hline$\left\|\nabla \cdot\left(\boldsymbol{u}^{n}-\boldsymbol{u}_{h}^{n}\right)\right\|_{\ell^{2}\left(L^{2}\right)}$ & 1.00 & 0.5 & 1.00 & 1 & 1.00 & 0 & 0.66 & $2 / 3$ \\
\hline
\end{tabular}

Table 10: Experimental $\delta t$ convergence rates for OSS-BE method using $Q 1 / Q 1$ spatial interpolation

\begin{tabular}{|c|c|c|c|c|c|c|c|c|}
\hline Variational Form & \multicolumn{2}{|c|}{$\mathrm{I}$} & \multicolumn{2}{|c|}{ II } & \multicolumn{2}{|c|}{ III } & \multicolumn{2}{|c|}{1} \\
\hline$\delta t$ vs. $h$ & & & $\delta t \sim$ & $.5 h$ & & & $\delta t \sim$ & $h^{1.5}$ \\
\hline & Num & Min & Num & Min & Num & Min & Num & Min \\
\hline$\left\|p^{n}-p_{h}^{n}\right\|_{\ell^{\infty}\left(L^{2}\right)}$ & 1.00 & 1 & 1.00 & 1 & 1.01 & 1 & 1.00 & 1 \\
\hline$\left\|\boldsymbol{u}^{n}-\boldsymbol{u}_{h}^{n}\right\|_{\ell \infty\left(L^{2}\right)}$ & 1.02 & 1 & 1.02 & 1 & 1.02 & 1 & 1.01 & 1 \\
\hline$\left\|\nabla\left(p^{n}-p_{h}^{n}\right)\right\|_{\ell^{2}\left(L^{2}\right)}$ & 1.00 & 0.5 & 1.00 & 0 & 1.00 & 1 & 0.66 & $2 / 3$ \\
\hline$\left\|\nabla \cdot\left(\boldsymbol{u}^{n}-\boldsymbol{u}_{h}^{n}\right)\right\|_{\ell^{2}\left(L^{2}\right)}$ & 1.00 & 0.5 & 1.00 & 1 & 1.00 & 0 & 0.66 & $2 / 3$ \\
\hline
\end{tabular}

Fig. 17 and Fig. 18 show the real and imaginary parts of the angular frequency ratio.

The plots comparing time marching schemes for the OSS method are very similar to the ones for ASGS and are not shown for succinctness. The fully discrete ASGS and OSS methods perform similarly according to this analysis, so we do not show a side by side comparison of both methods.

\section{Numerical results}

In this section we present two sets of numerical results. First, convergence tests are presented and compared with the predicted convergence rates obtained from convergence analysis. Then, numerical solutions of a wave propagation problem are compared in order to see the differences of the fully discrete methods.

\subsection{Convergence tests}

Let us consider a two dimensional transient problem with analytical solution to investigate the convergence properties of the stabilized $\mathrm{FE}$ formulations proposed. The spatial domain is the unit square $(0,1) \times(0,1)$ and the temporal domain is $(0,1)$. The forcing terms $\left[f_{p}, \boldsymbol{f}_{u}\right]$ are chosen such that the exact solution is $p=\sin (\pi x) \sin (\pi y) \cos \left(\frac{\pi}{3} t\right)$ and $\boldsymbol{u}=[p, p]$ with $\mu_{p}=\mu_{u}=1$. On the boundary we prescribe $p=0\left(\Gamma=\Gamma_{p}\right)$. The initial condition is taken as the exact solution at $t=0$. Various mesh sizes and time step sizes have been used to generate the results. We have used isotropic bilinear $(Q 1)$ and biquadratic $(Q 2)$ meshes of sizes $h=0.05,0.025,0.01,0.005$ and 0.002 . The stabilization algorithmic constant $C_{\tau}$ has been taken as 0.05 for $Q 1$ elements and 0.4 for $Q 2$ elements. The the length scale of the problem has been taken as $\mathrm{L}_{0}=\sqrt[d]{\operatorname{meas}(\Omega)}=1$.

In Tables $9 \sqrt{20}$ we show the convergence rates with respect to the time step size $\delta t$ for various stabilization methods (ASGS and OSS), time integrators (BE/CN/BDF2) and spatial interpolations $(Q 1$ and $Q 2)$. The numerical experiments have been carried out modifying the time step size and the mesh size at the same time. The relationship $(\delta t, h)$ is shown in each table for each variational form with the respective proportionality constant and power, $\delta t \sim C_{s} h^{s}$. The power constant $s$ has been chosen in two ways, first as $s=1$ for all the variational forms and, secondly, it has been chosen such that the best convergence is achieved for equal interpolation of the unknowns $(k=l)$; see Tables 9 20. It is observed than in all cases the numerical rate of convergence obtained (Num) is greater than or equal to the minimum one predicted by the convergence analysis (Min). Note that in some cases a clear superconvergence phenomenon is observed. 
Table 11: Experimental $\delta t$ convergence rates for ASGS-BE method using $Q 2 / Q 2$ spatial interpolation

\begin{tabular}{|c||c|c|c|c|c|c|c|c|c|c|c|c|c|c|}
\hline \multicolumn{1}{|c||}{ Variational Form } & \multicolumn{3}{|c|}{ I } & \multicolumn{3}{c|}{ II } & \multicolumn{3}{c|}{ III } & \multicolumn{3}{c|}{ I } & \multicolumn{3}{c|}{ II } & \multicolumn{3}{c|}{ III } \\
\hline$\delta t$ vs. $h$ & \multicolumn{4}{|c|}{$\delta t \sim 894 h^{2.5}$} & \multicolumn{2}{c|}{$\delta t \sim 40 h^{2}$} & \multicolumn{2}{c|}{$\delta t \sim 40 h^{2}$} \\
\hline \hline$\left\|p^{n}-p_{h}^{n}\right\|_{\ell^{\infty}\left(L^{2}\right)}$ & 0.98 & 1 & 0.98 & 1 & 0.98 & 1 & 0.99 & 1 & 0.99 & 1 & 0.99 & 1 \\
\hline$\left\|\boldsymbol{u}^{n}-\boldsymbol{u}_{h}^{n}\right\|_{\ell^{\infty}\left(L^{2}\right)}$ & 0.99 & 1 & 0.99 & 1 & 0.99 & 1 & 1.00 & 1 & 1.00 & 1 & 1.00 & 1 \\
\hline$\left\|\nabla\left(p^{n}-p_{h}^{n}\right)\right\|_{\ell^{2}\left(L^{2}\right)}$ & 0.99 & 0.5 & 0.99 & 0 & 0.99 & 1 & 0.98 & $4 / 5$ & 0.50 & 0.5 & 1.00 & 1 \\
\hline$\left\|\nabla \cdot\left(\boldsymbol{u}^{n}-\boldsymbol{u}_{h}^{n}\right)\right\|_{\ell^{2}\left(L^{2}\right)}$ & 0.99 & 0.5 & 0.99 & 1 & 0.99 & 0 & 0.99 & $4 / 5$ & 1.00 & 1 & 0.50 & 0.5 \\
\hline
\end{tabular}

Table 12: Experimental $\delta t$ convergence rates for OSS-BE method using $Q 2 / Q 2$ spatial interpolation

\begin{tabular}{|c||c|c|c|c|c|c|c|c|c|c|c|c|c|c|}
\hline \multicolumn{1}{|c||}{ Variational Form } & \multicolumn{3}{|c|}{ I } & \multicolumn{3}{c|}{ II } & \multicolumn{3}{c|}{ III } & \multicolumn{3}{c|}{ I } & \multicolumn{3}{c|}{ II } & \multicolumn{3}{c|}{ III } \\
\hline$\delta t$ vs. $h$ & \multicolumn{4}{|c|}{$\delta t \sim 894 h^{2.5}$} & \multicolumn{2}{c|}{$\delta t \sim 40 h^{2}$} & \multicolumn{2}{c|}{$\delta t \sim 40 h^{2}$} \\
\hline \hline$\left\|p^{n}-p_{h}^{n}\right\|_{\ell^{\infty}\left(L^{2}\right)}$ & 0.98 & 1 & 0.98 & 1 & 0.98 & 1 & 0.99 & 1 & 0.99 & 1 & 0.99 & 1 \\
\hline$\left\|\boldsymbol{u}^{n}-\boldsymbol{u}_{h}^{n}\right\|_{\ell^{\infty}\left(L^{2}\right)}$ & 0.99 & 1 & 0.99 & 1 & 0.99 & 1 & 1.00 & 1 & 1.00 & 1 & 1.00 & 1 \\
\hline$\left\|\nabla\left(p^{n}-p_{h}^{n}\right)\right\|_{\ell^{2}\left(L^{2}\right)}$ & 0.99 & 0.5 & 1.02 & 0 & 0.99 & 1 & 0.98 & $4 / 5$ & 0.51 & 0.5 & 1.00 & 1 \\
\hline$\left\|\nabla \cdot\left(\boldsymbol{u}^{n}-\boldsymbol{u}_{h}^{n}\right)\right\|_{\ell^{2}\left(L^{2}\right)}$ & 0.99 & 0.5 & 0.99 & 1 & 1.03 & 0 & 0.99 & $4 / 5$ & 0.99 & 1 & 0.52 & 0.5 \\
\hline
\end{tabular}

Table 13: Experimental $\delta t$ convergence rates for ASGS-CN method using $Q 1 / Q 1$ spatial interpolation

\begin{tabular}{|c||c|c|c|c|c|c|c|c|c|c|c|c|c|c|}
\hline \multicolumn{1}{|c|}{ Variational Form } & \multicolumn{3}{|c|}{ I } & \multicolumn{3}{c|}{ II } & \multicolumn{3}{c|}{ III } & \multicolumn{3}{c|}{ I } & \multicolumn{3}{c|}{ II } & \multicolumn{3}{c|}{ III } \\
\hline \multicolumn{1}{|c||}{$\delta t$ vs. $h$} & Num & Min & Num & Min & Num & Min & Num & Min & Num & Min & Num & Min \\
\hline \hline$\left\|p^{n}-p_{h}^{n}\right\|_{\ell^{\infty}\left(L^{2}\right)}$ & 2.00 & 1.5 & 2.00 & 1 & 2.00 & 1 & 2.60 & 2 & 3.38 & 2 & 3.39 & 2 \\
\hline$\left\|\boldsymbol{u}^{n}-\boldsymbol{u}_{h}^{n}\right\|_{\ell^{\infty}\left(L^{2}\right)}$ & 2.00 & 1.5 & 2.00 & 1 & 2.00 & 1 & 2.64 & 2 & 3.72 & 2 & 3.72 & 2 \\
\hline$\left\|\nabla\left(p^{n}-p_{h}^{n}\right)\right\|_{\ell^{2}\left(L^{2}\right)}$ & 1.00 & 1 & 1.00 & 0 & 1.00 & 1 & 1.33 & $4 / 3$ & 1.99 & 0 & 1.99 & 2 \\
\hline$\left\|\nabla \cdot\left(\boldsymbol{u}^{n}-\boldsymbol{u}_{h}^{n}\right)\right\|_{\ell^{2}\left(L^{2}\right)}$ & 1.00 & 1 & 1.00 & 1 & 1.00 & 0 & 1.33 & $4 / 3$ & 1.99 & 2 & 1.99 & 0 \\
\hline
\end{tabular}

Table 14: Experimental $\delta t$ convergence rates for OSS-CN method using $Q 1 / Q 1$ spatial interpolation

\begin{tabular}{|c||c|c|c|c|c|c|c|c|c|c|c|c|c|c|}
\hline \multicolumn{1}{|c||}{ Variational Form } & \multicolumn{3}{|c|}{ I } & \multicolumn{3}{c|}{ II } & \multicolumn{3}{c|}{ III } & \multicolumn{3}{c|}{ I } & \multicolumn{3}{c|}{ II } & \multicolumn{3}{c|}{ III } \\
\hline \multicolumn{1}{|c|}{$\delta t$ vs. $h$} & Num & Min & Num & Min & Num & Min & Num & Min & Num & Min & Num & Min \\
\hline \hline$\left\|p^{n}-p_{h}^{n}\right\|_{\ell^{\infty}\left(L^{2}\right)}$ & 2.00 & 1.5 & 2.00 & 1 & 2.00 & 1 & 2.60 & 2 & 3.38 & 2 & 3.39 & 2 \\
\hline$\left\|\boldsymbol{u}^{n}-\boldsymbol{u}_{h}^{n}\right\|_{\ell \infty\left(L^{2}\right)}$ & 2.00 & 1.5 & 2.00 & 1 & 2.00 & 1 & 2.64 & 2 & 3.72 & 2 & 3.72 & 2 \\
\hline$\left\|\nabla\left(p^{n}-p_{h}^{n}\right)\right\|_{\ell^{2}\left(L^{2}\right)}$ & 1.00 & 1 & 1.00 & 0 & 1.00 & 1 & 1.33 & $4 / 3$ & 1.99 & 0 & 1.99 & 2 \\
\hline$\left\|\nabla \cdot\left(\boldsymbol{u}^{n}-\boldsymbol{u}_{h}^{n}\right)\right\|_{\ell^{2}\left(L^{2}\right)}$ & 1.00 & 1 & 1.00 & 1 & 1.00 & 0 & 1.33 & $4 / 3$ & 1.99 & 2 & 1.99 & 0 \\
\hline
\end{tabular}

Table 15: Experimental $\delta t$ convergence rates for ASGS-CN method using $Q 2 / Q 2$ spatial interpolation

\begin{tabular}{|c||c|c|c|c|c|c|c|c|}
\hline \multicolumn{1}{|c||}{ Variational Form } & \multicolumn{3}{c|}{ I } & \multicolumn{3}{c|}{ II } & \multicolumn{3}{c|}{ III } & \multicolumn{3}{c|}{ I } \\
\hline \multicolumn{1}{|c||}{$\delta t$ vs. $h$} & \multicolumn{9}{|c|}{$\delta t \sim 0.5 h$} & \multicolumn{1}{c|}{$\delta t \sim 2 h^{1.25}$} \\
\hline \multicolumn{1}{|||}{} & Num & Min & Num & Min & Num & Min & Num & Min \\
\hline \hline$\left\|p^{n}-p_{h}^{n}\right\|_{\ell^{\infty}\left(L^{2}\right)}$ & 2.01 & 2 & 2.00 & 2 & 2.00 & 2 & 2.00 & 2 \\
\hline$\left\|\boldsymbol{u}^{n}-\boldsymbol{u}_{h}^{n}\right\|_{\ell^{\infty}\left(L^{2}\right)}$ & 2.26 & 2 & 2.00 & 2 & 2.00 & 2 & 2.13 & 2 \\
\hline$\left\|\nabla\left(p^{n}-p_{h}^{n}\right)\right\|_{\ell^{2}\left(L^{2}\right)}$ & 1.99 & 1.5 & 1.00 & 1 & 2.00 & 2 & 1.60 & 1.6 \\
\hline$\left\|\nabla \cdot\left(\boldsymbol{u}^{n}-\boldsymbol{u}_{h}^{n}\right)\right\|_{\ell^{2}\left(L^{2}\right)}$ & 1.99 & 1.5 & 2.00 & 2 & 1.00 & 1 & 1.60 & 1.6 \\
\hline
\end{tabular}


Table 16: Experimental $\delta t$ convergence rates for OSS-CN method using $Q 2 / Q 2$ spatial interpolation

\begin{tabular}{|c|c|c|c|c|c|c|c|c|}
\hline Variational Form & \multicolumn{2}{|c|}{ I } & \multicolumn{2}{|c|}{ II } & \multicolumn{2}{|c|}{ III } & \multicolumn{2}{|c|}{ I } \\
\hline$\delta t$ vs. $h$ & & & $\delta t \sim$ & $.5 h$ & & & $\delta t \sim$ & 1.25 \\
\hline & Num & Min & Num & Min & Num & Min & Num & Min \\
\hline$\left\|p^{n}-p_{h}^{n}\right\|_{\ell \infty\left(L^{2}\right)}$ & 2.01 & 2 & 21.99 & 2 & 2.00 & 2 & 2.00 & 2 \\
\hline $\boldsymbol{u}^{n}-\boldsymbol{u}_{h}^{n} \|_{\ell^{\infty}\left(L^{2}\right)}$ & 2.04 & 2 & 1.99 & 2 & 2.03 & 2 & 2.00 & 2 \\
\hline$\left\|\nabla\left(p^{n}-p_{h}^{n}\right)\right\|_{\ell^{2}\left(L^{2}\right)}$ & 2.00 & 1.5 & 1.00 & 1 & 2.00 & 2 & 1.60 & 1.6 \\
\hline$\left\|\nabla \cdot\left(\boldsymbol{u}^{n}-\boldsymbol{u}_{h}^{n}\right)\right\|_{\ell^{2}\left(L^{2}\right)}$ & 2.02 & 1.5 & 1.98 & 2 & 1.02 & 1 & 1.62 & 1.6 \\
\hline
\end{tabular}

Table 17: Experimental $\delta t$ convergence rates for ASGS-BDF2 method using $Q 1 / Q 1$ spatial interpolation

\begin{tabular}{|c|c|c|c|c|c|c|c|c|c|c|c|c|}
\hline Variational Form & \multicolumn{2}{|c|}{ I } & & & \multirow{2}{*}{\multicolumn{2}{|c|}{ III }} & \multicolumn{2}{|c|}{1} & \multicolumn{2}{|c|}{ II } & \multicolumn{2}{|c|}{ III } \\
\hline$\delta t$ vs. $h$ & & & $\delta t \sim$ & $.5 h$ & & & $\delta t \sim 0$ & $h^{0.75}$ & $\delta t \sim$ & $1 h^{0.5}$ & $\delta t \sim$ & $1 h^{0.5}$ \\
\hline & Num & Min & Num & Min & Num & Min & Num & Min & Num & Min & Num & Min \\
\hline$\left\|p^{n}-p_{h}^{n}\right\|_{\ell \infty\left(L^{2}\right)}$ & 2.00 & 1.5 & 2.00 & 1 & 2.00 & 1 & 2.66 & 2 & 3.93 & 2 & 3.93 & 2 \\
\hline$\left\|\boldsymbol{u}^{n}-\boldsymbol{u}_{h}^{n}\right\|_{\ell^{\infty}\left(L^{2}\right)}$ & 2.00 & 1.5 & 2.00 & 1 & 2.00 & 1 & 2.67 & 2 & 4.04 & 2 & 4.04 & 2 \\
\hline$\nabla\left(p^{n}-p_{h}^{n}\right) \|_{\ell^{2}\left(L^{2}\right)}$ & 1.00 & 1 & 1.00 & 0 & 1.00 & 1 & 1.32 & $4 / 3$ & 1.99 & 0 & 1.99 & 2 \\
\hline$\left\|\nabla \cdot\left(\boldsymbol{u}^{n}-\boldsymbol{u}_{h}^{n}\right)\right\|_{\ell^{2}\left(L^{2}\right)}$ & 1.00 & 1 & 1.00 & 1 & 1.00 & 0 & 1.32 & $4 / 3$ & 1.99 & 2 & 1.99 & 0 \\
\hline
\end{tabular}

Table 18: Experimental $\delta t$ convergence rates for OSS-BDF2 method using $Q 1 / Q 1$ spatial interpolation

\begin{tabular}{|c||c|c|c|c|c|c|c|c|c|c|c|c|c|}
\hline \multicolumn{1}{|c|}{ Variational Form } & \multicolumn{3}{|c|}{ I } & \multicolumn{3}{c|}{ II } & \multicolumn{3}{c|}{ III } & \multicolumn{3}{c|}{ I } & \multicolumn{3}{c|}{ II } & \multicolumn{3}{c|}{ III } \\
\hline \multicolumn{1}{|c||}{$\delta t$ vs. $h$} & Num & Min & Num & Min & Num & Min & Num & Min & Num & Min & Num & Min \\
\hline \hline$\left\|p^{n}-p_{h}^{n}\right\|_{\ell^{\infty}\left(L^{2}\right)}$ & 2.00 & 1.5 & 2.00 & 1 & 2.00 & 1 & 2.66 & 2 & 3.93 & 2 & 3.93 & 2 \\
\hline$\left\|\boldsymbol{u}^{n}-\boldsymbol{u}_{h}^{n}\right\|_{\ell^{\infty}\left(L^{2}\right)}$ & 2.00 & 1.5 & 2.00 & 1 & 2.00 & 1 & 2.67 & 2 & 4.04 & 2 & 4.04 & 2 \\
\hline$\left\|\nabla\left(p^{n}-p_{h}^{n}\right)\right\|_{\ell^{2}\left(L^{2}\right)}$ & 1.00 & 1 & 1.00 & 0 & 1.00 & 1 & 1.32 & $4 / 3$ & 1.99 & 0 & 1.99 & 2 \\
\hline$\left\|\nabla \cdot\left(\boldsymbol{u}^{n}-\boldsymbol{u}_{h}^{n}\right)\right\|_{\ell^{2}\left(L^{2}\right)}$ & 1.00 & 1 & 1.00 & 1 & 1.00 & 0 & 1.32 & $4 / 3$ & 1.99 & 2 & 1.99 & 0 \\
\hline
\end{tabular}

Table 19: Experimental $\delta t$ convergence rates for ASGS-BDF2 method using $Q 2 / Q 2$ spatial interpolation

\begin{tabular}{|c|c|c|c|c|c|c|c|c|}
\hline Variational Form & \multicolumn{2}{|c|}{ I } & \multicolumn{2}{|c|}{ II } & \multicolumn{2}{|c|}{ III } & \multirow{2}{*}{\multicolumn{2}{|c|}{$\begin{array}{c}\mathrm{I} \\
\delta t \sim 2 h^{1.25}\end{array}$}} \\
\hline$\delta t$ vs. $h$ & & & $\delta t \sim$ & $.5 h$ & & & & \\
\hline & Num & Min & Num & Min & Num & Min & Num & Min \\
\hline$\left\|p^{n}-p_{h}^{n}\right\|_{\ell \infty\left(L^{2}\right)}$ & 2.17 & 2 & 2.00 & 2 & 2.13 & 2 & 2.41 & 2 \\
\hline $\boldsymbol{u}^{n}-\boldsymbol{u}_{h}^{n} \|_{\ell \infty\left(L^{2}\right)}$ & 2.19 & 2 & 2.00 & 2 & 2.00 & 2 & 2.34 & 2 \\
\hline$\nabla\left(p^{n}-p_{h}^{n}\right) \|_{\ell^{2}\left(L^{2}\right)}$ & 1.99 & 1.5 & 1.00 & 1 & 2.00 & 2 & 1.59 & 1.6 \\
\hline$\left\|\nabla \cdot\left(\boldsymbol{u}^{n}-\boldsymbol{u}_{h}^{n}\right)\right\|_{\ell^{2}\left(L^{2}\right)}$ & 1.99 & 1.5 & 2.00 & 2 & 1.00 & 1 & 1.59 & 1.6 \\
\hline
\end{tabular}

Table 20: Experimental $\delta t$ convergence rates for OSS-BDF2 method using $Q 2 / Q 2$ spatial interpolation

\begin{tabular}{|c||c|c|c|c|c|c|c|c|}
\hline \multicolumn{1}{|c||}{ Variational Form } & \multicolumn{3}{|c|}{ I } & \multicolumn{3}{c|}{ II } & \multicolumn{3}{c|}{ III } & \multicolumn{3}{c|}{ I } \\
\hline \multicolumn{1}{|c||}{$\delta t$ vs. $h$} & \multicolumn{9}{|c|}{$\delta t \sim 0.5 h$} & \multicolumn{3}{c|}{$\sim 2 h^{1.25}$} \\
\hline & Num & Min & Num & Min & Num & Min & Num & Min \\
\hline \hline$\left\|p^{n}-p_{h}^{n}\right\|_{\ell^{\infty}\left(L^{2}\right)}$ & 2.18 & 2 & 2.02 & 2 & 2.14 & 2 & 2.42 & 2 \\
\hline$\left\|\boldsymbol{u}^{n}-\boldsymbol{u}_{h}^{n}\right\|_{\ell^{\infty}\left(L^{2}\right)}$ & 2.03 & 2 & 2.00 & 2 & 2.03 & 2 & 2.24 & 2 \\
\hline$\left\|\nabla\left(p^{n}-p_{h}^{n}\right)\right\|_{\ell^{2}\left(L^{2}\right)}$ & 2.00 & 1.5 & 1.01 & 1 & 2.00 & 2 & 1.60 & 1.6 \\
\hline$\left\|\nabla \cdot\left(\boldsymbol{u}^{n}-\boldsymbol{u}_{h}^{n}\right)\right\|_{\ell^{2}\left(L^{2}\right)}$ & 2.02 & 1.5 & 2.00 & 2 & 1.03 & 1 & 1.64 & 1.6 \\
\hline
\end{tabular}




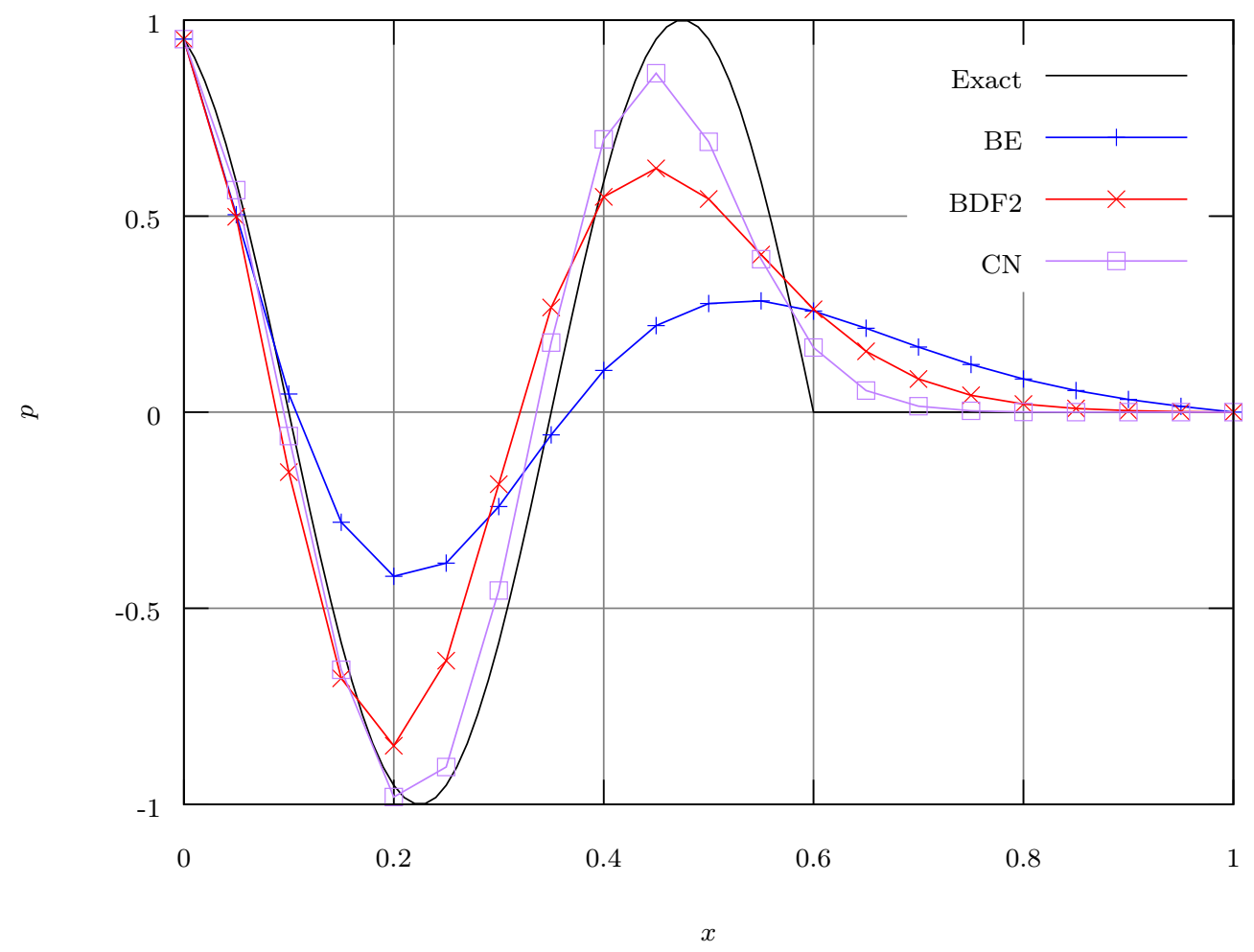

Figure 19: Numerical solution using the ASGS formulation and different time marching schemes

\subsection{Numerical comparison}

In Section 4 we analyzed dispersion and dissipation analytically through Fourier techniques. We present now a simple test to verify experimentally the predictions of the Fourier analysis. Let us consider a 1D problem in $\Omega=(0, L)=(0,1)$ and $\Upsilon=(0,0.8)$, and let us solve the mixed wave equation with $\mu_{p}=1$, $\mu_{u}=1$, zero initial conditions and boundary conditions $p(0, t)=\sin (\omega t)$ and $p(L, t)=0$. We compare the solutions obtained with the fully discrete methods at $t=0.6$.

For $\omega=10 \pi$ a quite coarse pair of mesh and time step sizes is $(h, \delta t)=(0.05,0.05)$. This allows us to see dispersion and dissipation in the numerical solution when compared with the exact $\operatorname{solution} \sin (\omega t-$ $k x)(1-H(x-c t))$, where $H$ is the Heaviside step function. The algorithmic constant is taken as $C_{\tau}=0.1$ and the elements used are P1.

Fig. 19 shows the numerical solutions obtained with ASGS and three time marching schemes. CN is the least dissipative while BE is the most dissipative and BDF2 is somewhere in the middle. These numerical results are in agreement with the previous Fourier analysis. OSS behaves similarly and we do not show those results. Figs. 20 22 compare the ASGS method with the OSS method for the same time integration scheme, showing a very similar numerical behavior.

\section{Conclusions}

In this work we have presented fully discrete methods arising from the combination of spatial discretization methods, namely stabilized FE methods, and temporal discretization methods (backward Euler, Crank-Nicolson and 2nd order backward differentiation formula) for the mixed wave equation in three different variational forms. The stabilization parameters have been designed such that they mimic the continuous setting. 


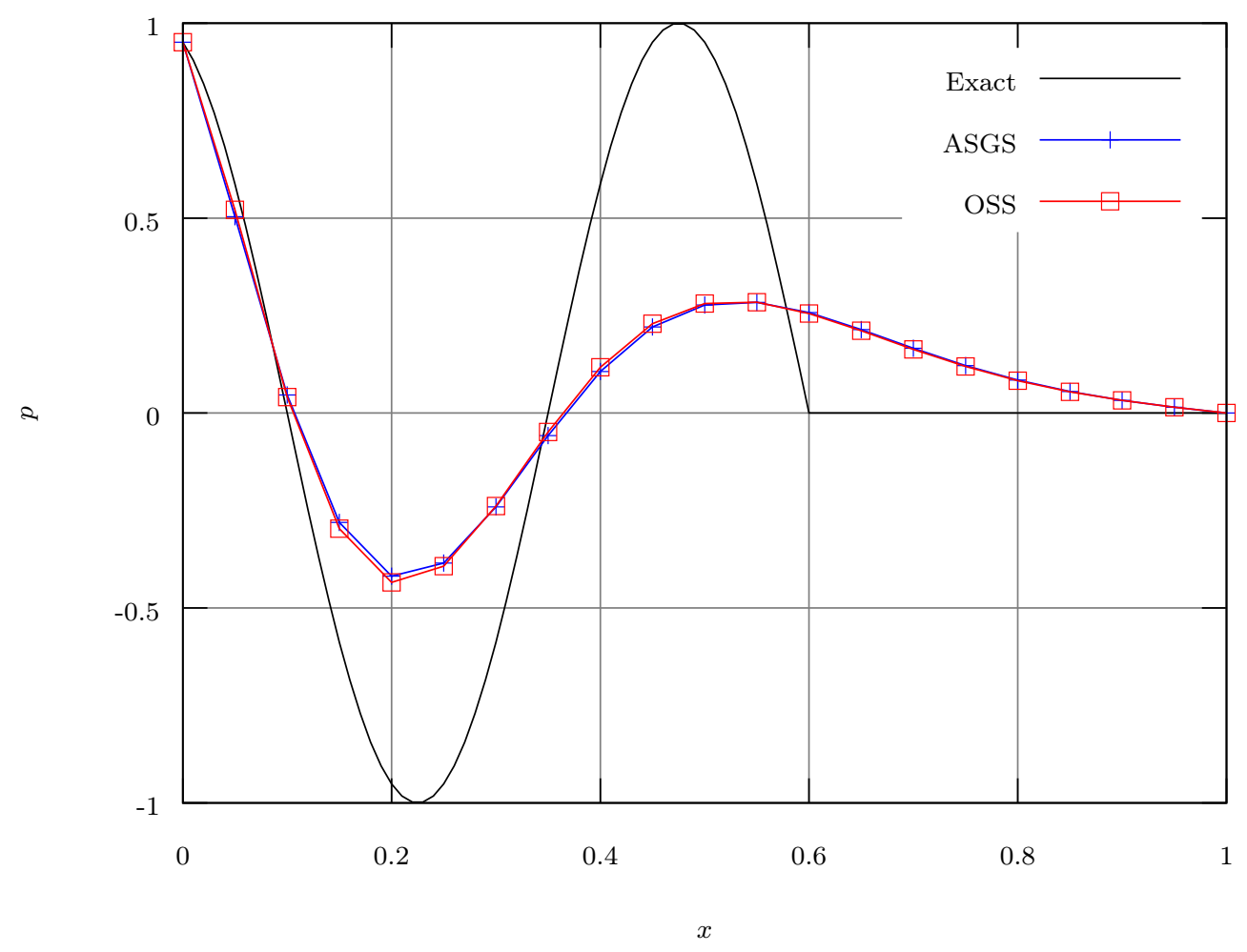

Figure 20: Comparison of ASGS and OSS using BE as time integration

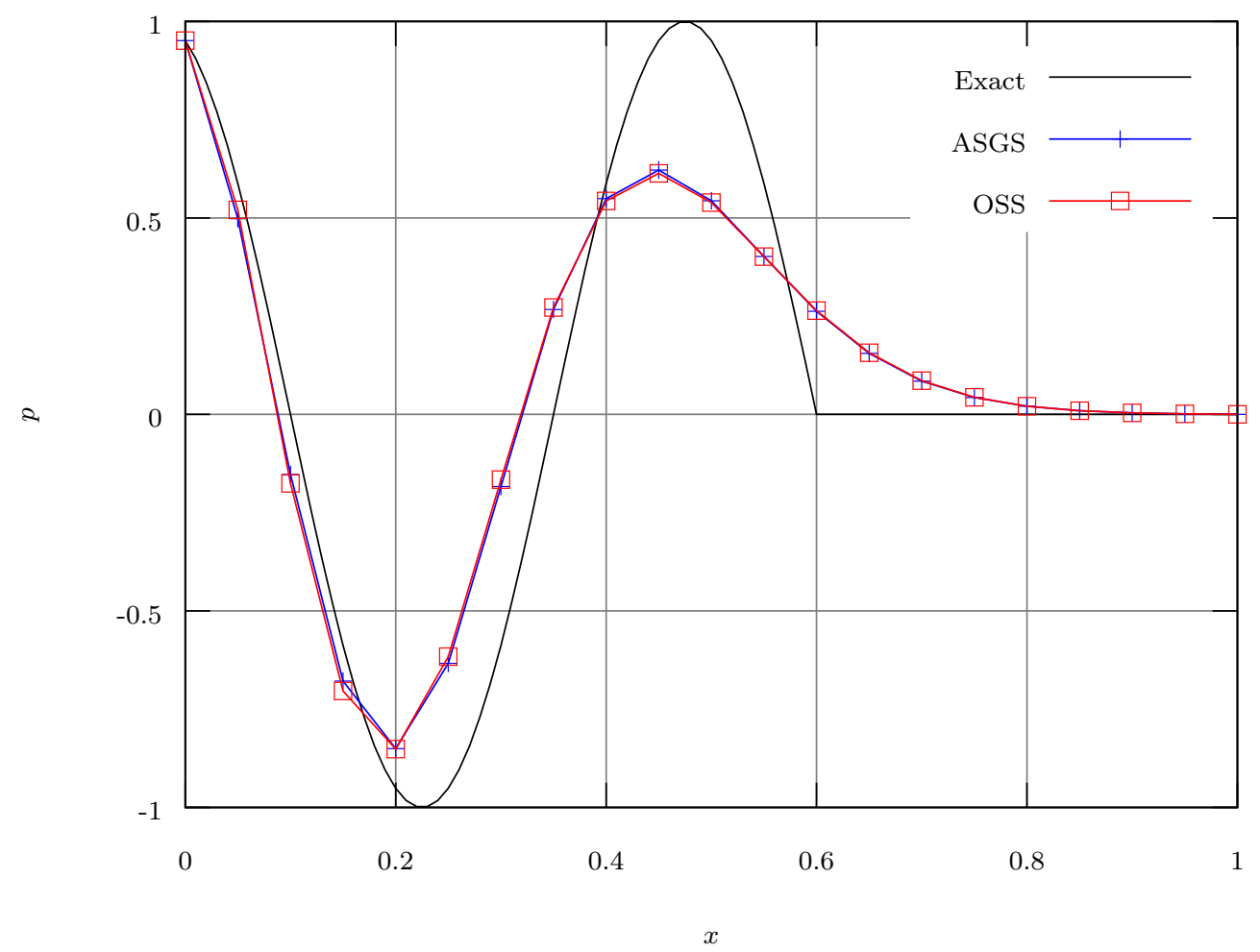

Figure 21: Comparison of ASGS and OSS using BDF2 as time integration 


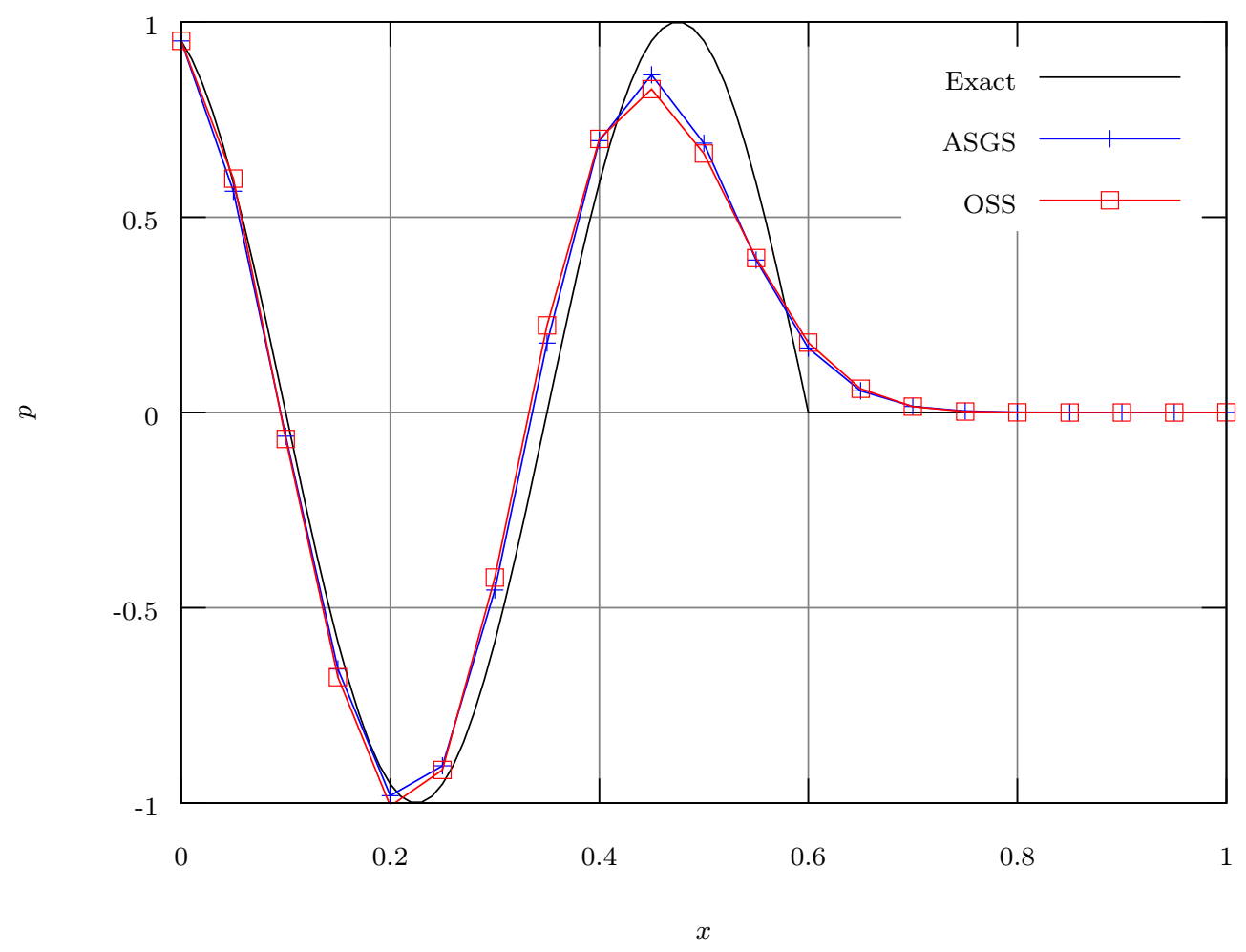

Figure 22: Comparison of ASGS and OSS using CN as time integration

Stability and convergence has been proved for all combinations of space discretization and time discretization. Stability, dispersion and dissipation of the fully discrete methods in 1D for equal interpolation of $[p, \boldsymbol{u}]$ has been analyzed using Fourier techniques. According to this analysis, CN performs better than $\mathrm{BE}$ and BDF2. Additionally, ASGS and OSS perform similarly.

Numerical convergence tests have been performed and the results obtained in the numerical experiments are in agreement with the theoretical predictions. Additionally, the fully discrete methods have been compared qualitatively. This comparison shows the differences in dispersion and dissipation of the methods and is in agreement with the Fourier analysis.

\section{Acknowledgments}

The work of the first author was funded by the Ministerio de Educación, Cultura y Deporte, Programa de Formación del Profesorado Universitario (FPU), in Spain, with grant AP2010-0563, the work of the second author by the FP7 Grant No. 308874 (project Eunison) and the ICREA Acadèmia Program from the Catalan Government, and the work of the third author was funded by the European Research Council under the FP7 Programme Ideas through the Starting Grant No. 258443 - COMFUS: Computational Methods for Fusion Technology.

\section{References}

[1] J. Berland, C. Bogey, C. Bailly, Low-dissipation and low-dispersion fourth-order runge-kutta algorithm, Computers \& Fluids 35 (2006) 1459 - 1463.

[2] K. Tselios, T. Simos, Runge-Kutta methods with minimal dispersion and dissipation for problems arising from computational acoustics, Journal of Computational and Applied Mathematics 175 (2005) 173 - 181.

[3] H. Xu, P. Sagaut, Optimal low-dispersion low-dissipation LBM schemes for computational aeroacoustics, Journal of Computational Physics 230 (2011) $5353-5382$. 
[4] J. Li, Z. Yang, The von neumann analysis and modified equation approach for finite difference schemes, Applied Mathematics and Computation 225 (2013) $610-621$

[5] G. Scovazzi, J. N. Shadid, E. Love, W. J. Rider, A conservative nodal variational multiscale method for Lagrangian shock hydrodynamics, Computer Methods in Applied Mechanics and Engineering 199 (2010) 3059-3100.

[6] J. Nordström, Conservative finite difference formulations, variable coefficients, energy estimates and artificial dissipation, Journal of Scientific Computing 29 (2006) 375-404.

[7] P. Joly, J. Rodríguez, Optimized higher order time discretization of second order hyperbolic problems: Construction and numerical study, Journal of Computational and Applied Mathematics 234 (2010) 1953 - 1961.

[8] Y. Sun, P. Tse, Symplectic and multisymplectic numerical methods for Maxwell's equations, Journal of Computational Physics 230 (2011) 2076-2094.

[9] R. Codina, Finite element approximation of the hyperbolic wave equation in mixed form, Computer Methods in Applied Mechanics and Engineering 197 (2008) 1305 - 1322.

[10] S. Badia, R. Codina, H. Espinoza, Stability, convergence, and accuracy of stabilized finite element methods for the wave equation in mixed form, SIAM Journal on Numerical Analysis 52 (2014) 1729-1752.

[11] H. Espinoza, R. Codina, S. Badia, A Sommerfeld non-reflecting boundary condition for the wave equation in mixed form, Computer Methods in Applied Mechanics and Engineering 276 (2014) $122-148$.

[12] S. Badia, R. Codina, Analysis of a stabilized finite element approximation of the transient convection-diffusion equation using an ALE framework, SIAM Journal on Numerical Analysis 44 (2006) 2159-2197.

[13] E. Burman, M. A. Fernández, Galerkin finite element methods with symmetric pressure stabilization for the transient Stokes equations: Stability and convergence analysis, SIAM Journal on Numerical Analysis 47 (2008) $409-439$.

[14] E. Burman, Consistent SUPG-method for transient transport problems: Stability and convergence, Computer Methods in Applied Mechanics and Engineering 199 (2010) $1114-1123$.

[15] T. Hughes, L. Franca, G. Hulbert, A new finite element formulation for computational fluid dynamics: VIII. The Galerkin / least-squares method for advective-diffusive equations, Computer Methods in Applied Mechanics and Engineering 73 (1989) 173-189.

[16] J. Lopez-Marcos, Crank-Nicolson method, in: Encyclopedia of Mathematics, Springer, 2012, p. 1.

[17] G. Noh, S. Ham, K.-J. Bathe, Performance of an implicit time integration scheme in the analysis of wave propagations, Computers \& Structures 123 (2013) 93-105.

[18] A. Idesman, H. Samajder, E. Aulisa, P. Seshaiyer, Benchmark problems for wave propagation in elastic materials, Computational Mechanics 43 (2009) 797-814.

[19] R. Codina, Analysis of a stabilized finite element approximation of the Oseen equations using orthogonal subscales, Applied Numerical Mathematics 58 (2008) $264-283$.

[20] T. J. Hughes, Multiscale phenomena: Green's functions, the Dirichlet-to-Neumann formulation, subgrid scale models, bubbles and the origins of stabilized methods, Computer Methods in Applied Mechanics and Engineering 127 (1995) 387 -401 .

[21] T. J. Hughes, G. R. Feijoo, L. Mazzei, J.-B. Quincy, The variational multiscale method-A paradigm for computational mechanics, Computer Methods in Applied Mechanics and Engineering 166 (1998) $3-24$.

[22] R. Codina, Stabilization of incompressibility and convection through orthogonal sub-scales in finite element methods, Computer Methods in Applied Mechanics and Engineering 190 (2000) 1579 - 1599.

[23] R. Codina, Stabilized finite element approximation of transient incompressible flows using orthogonal subscales, Computer Methods in Applied Mechanics and Engineering 191 (2002) 4295 - 4321.

[24] R. Codina, J. Principe, O. Guasch, S. Badia, Time dependent subscales in the stabilized finite element approximation of incompressible flow problems, Computer Methods in Applied Mechanics and Engineering 196 (2007) 2413 - 2430.

[25] S. Badia, R. Codina, On a multiscale approach to the transient Stokes problem: Dynamic subscales and anisotropic space-time discretization, Applied Mathematics and Computation 207 (2009) 415 - 433.

[26] S. Badia, R. Codina, Unified stabilized finite element formulations for the Stokes and the Darcy problems, SIAM Journal on Numerical Analysis 47 (2009) 1971-2000.

[27] A.-S. Bonnet-BenDhia, P. Ciarlet, C. Zwölf, Time harmonic wave diffraction problems in materials with sign-shifting coefficients, Journal of Computational and Applied Mathematics 234 (2010) 1912-1919.

[28] A.-S. Bonnet-BenDhia, L. Chesnel, H. Haddar, On the use of T-coercivity to study the interior transmission eigenvalue problem, Comptes Rendus Mathematique 349 (2011) 647-651.

[29] L. Chesnel, P. Ciarlet, T-coercivity and continuous galerkin methods: application to transmission problems with sign changing coefficients, Numerische Mathematik 124 (2013) 1-29.

[30] A.-S. Bonnet-BenDhia, L. Chesnel, P. Ciarlet, T-coercivity for the Maxwell problem with sign-changing coefficients, Communications in Partial Differential Equations 39 (2014) 1007-1031.

[31] J.-L. Guermond, R. Pasquetti, A correction technique for the dispersive effects of mass lumping for transport problems, Computer Methods in Applied Mechanics and Engineering 253 (2013) 186-198. 